\title{
Observations of the Sun at Vacuum-Ultraviolet Wavelengths from Space. Part II: Results and Interpretations
}

\author{
Klaus Wilhelm • Eckart Marsch • Bhola N. Dwivedi • \\ Uri Feldman
}

Received: 22 February 2007 / Accepted: 9 October 2007 / Published online: 9 January 2008

(C) Springer Science+Business Media B.V. 2008

\begin{abstract}
In Part I of this review, the concepts of solar vacuum-ultraviolet (VUV) observations were outlined together with a discussion of the space instrumentation used for the investigations. A section on spectroradiometry provided some quantitative results on the solar VUV radiation without considering any details of the solar phenomena leading to the radiation. Here, in Part II, we present solar VUV observations over the last decades and their interpretations in terms of the plasma processes and the parameters of the solar atmosphere, with emphasis on the spatial and thermal structures of the chromosphere, transition region and corona of the quiet Sun. In addition, observations of active regions, solar flares and prominences are included as well as of small-scale events. Special sections are devoted to the elemental composition of the solar atmosphere and theoretical considerations on the heating of the corona and the generation of the solar wind.
\end{abstract}

Keywords Solar VUV—observations and interpretations · Solar atmosphere · Solar plasma processes and parameters

\section{Abbreviations \\ ACE Advanced Composition Explorer \\ BCS Bragg Crystal Spectrometer}

K. Wilhelm ( $\varangle) \cdot$ E. Marsch · B.N. Dwivedi

Max-Planck-Institut für Sonnensystemforschung (MPS), Max-Planck-Str. 2, 37191 Katlenburg-Lindau, Germany

e-mail: wilhelm@mps.mpg.de

B.N. Dwivedi

Department of Applied Physics, Institute of Technology, Banaras Hindu University, Varanasi 221005, India

U. Feldman

Artep Inc., 2922 Excelsior Spring Ct., Ellicott City, MA 21042, USA

U. Feldman

E.O. Hulburt Center for Space Research, Naval Research Laboratory (NRL), Washington,

DC 20375-5352, USA 


\begin{tabular}{|c|c|}
\hline CDS & Coronal Diagnostic Spectrometer \\
\hline $\mathrm{CI}$ & Carbonaceous Ivuna \\
\hline CHASE & Coronal Helium Abundance Spacelab Experiment \\
\hline CME & Coronal Mass Ejection \\
\hline DEM & Differential Emission Measure \\
\hline EIT & Extreme-ultraviolet Imaging Telescope \\
\hline EUV & Extreme Ultraviolet \\
\hline FIP & First Ionization Potential \\
\hline FUSE & Far-Ultraviolet Spectroscopic Explorer \\
\hline GIS & Grazing-Incidence Spectrometer \\
\hline $\mathrm{HCO}$ & Harvard College Observatory \\
\hline HRTS & High-Resolution Telescope and Spectrograph \\
\hline ISSI & International Space Science Institute \\
\hline IUE & International Ultraviolet Explorer \\
\hline JOP & Joint Observing Programme \\
\hline KPNSO & Kitt Peak National Solar Observatory \\
\hline LASCO & Large Angle Spectroscopic Coronagraph \\
\hline LASP & Laboratory for Atmospheric and Space Physics \\
\hline LOS & Line of Sight \\
\hline MDI & Michelson Doppler Imager \\
\hline MHD & Magneto-Hydrodynamics \\
\hline MICA & Mirror Coronagraph for Argentina \\
\hline MPS & Max-Planck-Institut für Sonnensystemforschung ${ }^{1}$ \\
\hline MSDP & Multichannel Subtractive Double Pass \\
\hline MSSTA & Multi-Spectral Solar Telescope Array \\
\hline NRL & Naval Research Laboratory \\
\hline NIXT & Normal-Incidence X-ray Telescope \\
\hline OSO & Orbiting Solar Observatory \\
\hline QLT & Quasi-Linear Theory \\
\hline RESIK & Rentgenovsky Spektrometr s Izognutymi Kristalami \\
\hline SEM & Solar Extreme-ultraviolet Monitor \\
\hline SERTS & Solar Extreme-ultraviolet Research Telescope and Spectrograph \\
\hline SMM & Solar Maximum Mission \\
\hline $\mathrm{SOHO}$ & Solar and Heliospheric Observatory \\
\hline SOLFLEX & Solar Flare X-rays \\
\hline SOLSTICE & Solar-Stellar Irradiance Comparison Experiment \\
\hline STEREO & Solar Terrestrial Relations Observatory \\
\hline SUMER & Solar Ultraviolet Measurements of Emitted Radiation \\
\hline SWICS & Solar Wind Ion Composition Spectrometer \\
\hline SXT & Soft X-ray Telescope \\
\hline THEMIS & $\begin{array}{l}\text { Telescopio Heliografico para el Estudio del Magnetismo y de las } \\
\text { Inestabilidades Solares }\end{array}$ \\
\hline TRACE & Transition Region and Coronal Explorer \\
\hline TRC & Transition Region Camera \\
\hline UCS & Ultraviolet Coronal Spectrometer \\
\hline UVCS & Ultraviolet Coronagraph Spectrometer \\
\hline
\end{tabular}

\footnotetext{
${ }^{1}$ Prior to 30 June 2004, Max-Planck-Institut für Aeronomie (MPAE).
} 


$\begin{array}{ll}\text { UVSP } & \text { Ultraviolet Spectrometer and Polarimeter } \\ \text { VAULT } & \text { Very high Angular-resolution Ultraviolet Telescope } \\ \text { VDF } & \text { Velocity Distribution Function } \\ \text { VLA } & \text { Very Large Array } \\ \text { VUV } & \text { Vacuum Ultraviolet } \\ \text { WKB } & \text { Wentzel-Kramers-Brillouin (method) }\end{array}$

\section{Introduction}

In Part I of this review (Wilhelm et al. 2004a), we summarized the early observations of the solar atmosphere in the vacuum-ultraviolet (VUV) wavelength range (10 nm to $200 \mathrm{~nm}$ ) followed by a discussion of the modern space instrumentation used over the last decades. Sections on spectral line formation, spectroscopic plasma diagnostics as well as radiance and irradiance measurements completed Part I. Here, in Part II, we present in more detail observations related to the different structures of the solar atmosphere and their dynamics. We place special emphasis on the heating of the corona and the acceleration of the solar wind. Most of the discussion is related to VUV measurements, but a few observations in the adjacent near-ultraviolet and X-ray ranges (cf., Table 1 of Part I) are mentioned, in particular, in relation to some aspects of flare dynamics (cf., Sect. 2.2.2.6). It is our objective to write this part in such a way that it can be read without necessarily consulting Part I. However, for brief descriptions of the instruments and basic formulae on plasma diagnostics, the reader is referred to Part I and its extensive list of references. The references given in this part of the review are mainly related to observational and theoretical studies. The progress made in this field of research during the last 25 years and the wealth of information thus obtained led to an enormous number of publications. About 600 of them are included in our list of references, but we realize that it is far from being exhaustive.

\section{VUV Observations of the Solar Atmosphere with Emphasis on Recent Results}

\subsection{Plasma Parameters of the Solar Atmosphere}

In this section we consider plasma parameters, such as the electron density, ${ }^{1} n_{\mathrm{e}}$, electron temperature, $T_{\mathrm{e}}$, and ion temperature, $T_{\mathrm{i}}$, as measured in the different regions of the solar atmosphere. It is difficult, if not impossible, to devise a fully consistent scheme of presenting the results (obtained by the many VUV instruments discussed in Part I). The reason is that the solar atmosphere is highly structured in temperature and density both in vertical and horizontal spatial dimensions, and is, moreover, highly variable with time. Therefore, we first adhere to the traditional way of dividing the solar atmosphere into the photosphere, the chromosphere, the transition region, and the corona. In the discussion that follows, it may thus appear that the solar atmosphere consists of four different layers one on top of each other, ordered mainly by their electron temperatures, which are roughly $5800 \mathrm{~K}$ to $4500 \mathrm{~K}$ in the photosphere from the solar "surface" to the temperature minimum, from there to $2.5 \times 10^{4} \mathrm{~K}$ in the chromosphere, followed by the transition region to the corona with temperatures of $T_{\mathrm{e}}>6 \times 10^{5} \mathrm{~K}$. This temperature profile is schematically illustrated in Fig. 1.

\footnotetext{
${ }^{1}$ The symbols used for physical quantities are compiled in Appendix B of Part I.
} 
Fig. 1 Schematic temperature gradient through the solar chromosphere and transition region (after Peter 2001), where the temperature first slowly increases above the temperature minimum, before it abruptly rises from $6 \times 10^{3} \mathrm{~K}$ to $1 \mathrm{MK}$. The ordinate on the right gives the plasma density. Several bright ultraviolet emission lines are indicated at their formation temperatures (cf., Part I, Sect. 2.2)

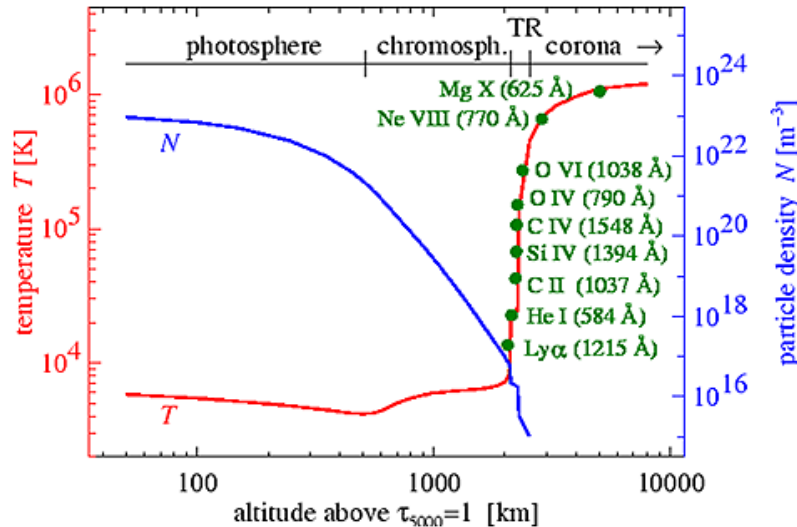

Clearly, such a subdivision into various layers does not provide a full picture. In quietSun and coronal-hole regions, the chromospheric and transition-region plasmas are usually confined to the first $10 \mathrm{Mm}$ above the limb (cf., Figs. 15 and 17 in Sect. 3). Nevertheless, plasmas with temperatures from $1 \times 10^{4} \mathrm{~K}$ to $1 \mathrm{MK}$ are detected along any line of sight (LOS) in this height range.

Phenomena or processes that operate in more than one regime, or for other reasons do not fit into the above scheme of layers, are discussed in subsequent sections and cross-references are given there. In some sense, this amounts to a discussion of the quiet-Sun atmosphere first. However, many processes to be presented later also operate in quiet-Sun regions, as well as in more active parts of the Sun.

\subsubsection{The Photosphere}

We review the thermal and spatial structures of the photosphere in the following two paragraphs. Observations of the photosphere in the VUV wavelength range are limited to the region near the temperature minimum that defines the interface between the photosphere and the chromosphere. Only this region is discussed here in some detail. The deeper atmospheric layers are opaque to VUV radiation.

2.1.1.1 The Thermal Structure In the VUV wavelength range, observations of the upper photosphere below the temperature minimum are possible above the $\mathrm{Si} \mathrm{I}{ }^{1} \mathrm{D}$ absorption edge at $168.2 \mathrm{~nm}$ and below the ionization limit of aluminium at $208 \mathrm{~nm}$ (Tousey 1964; Widing et al. 1970). This range is characterized by a decrease of about two orders of magnitude of the spectral radiance of the continuum towards shorter wavelengths and a rapid decrease of the brightness temperature shortwards of $210 \mathrm{~nm}$ (Samain et al. 1975; Brueckner et al. 1976). As a consequence, the Fraunhofer lines fade out. The last Fraunhofer line that can be identified with certainty is at $\approx 170 \mathrm{~nm}$ (Detwiler et al. 1961a). In parallel, emission lines become more and more prominent.

The continuum near $160 \mathrm{~nm}$, longwards of the Si I ${ }^{3} \mathrm{P}$ edges between $152.1 \mathrm{~nm}$ and $152.6 \mathrm{~nm}$, probably originates in the coolest plasma regions of the Sun that contribute to VUV radiation (Detwiler et al. 1961b; Widing et al. 1970; Brueckner and Moe 1972; Carver et al. 1972; Vernazza et al. 1981). Compared to the early results obtained by these authors, the actual measurements of the continuum radiance at $156 \mathrm{~nm}$ in quiet-Sun areas give values that are a factor of about two less (see, e.g., the well-calibrated observations by Brekke and 
Fig. 2 The upper photosphere of the quiet Sun observed by TRACE in the $170 \mathrm{~nm}$ passband in two subfields on 14 October 1998, at 10:05 UTC. The spatial scales are in seconds of arc from the centre of the disk. Incomplete data caused the stripes on the right-hand side (Fig. 3 of Krijger et al. 2001)

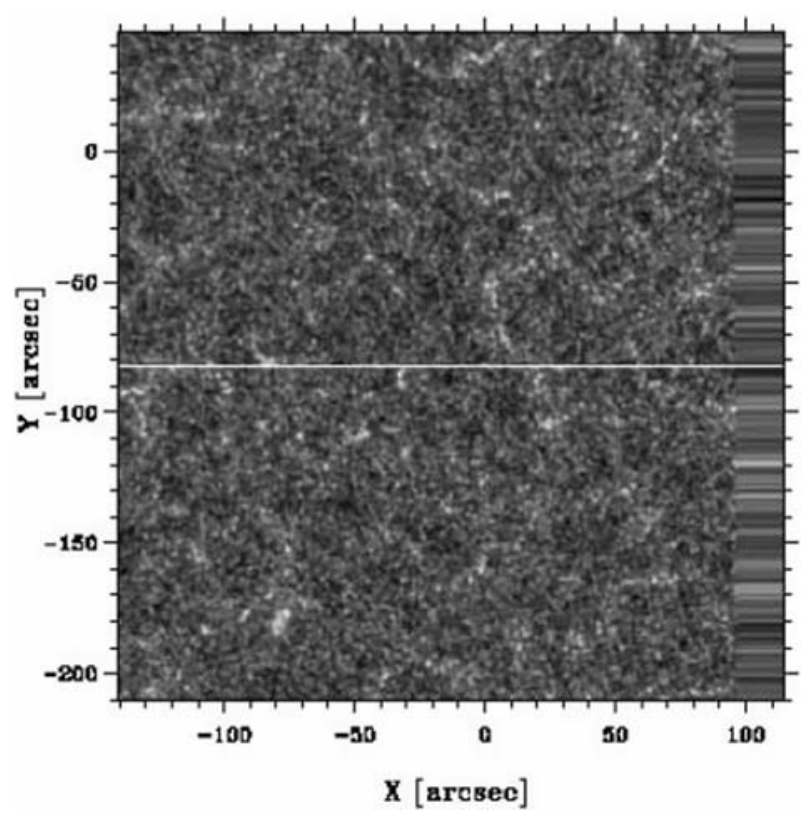

Kjeldseth-Moe 1994; Wilhelm et al. 1999). The results yield a minimum brightness temperature between $4450 \mathrm{~K}$ and $4520 \mathrm{~K}$ at the top of the photosphere, in good agreement with the value of $4430 \mathrm{~K} \pm 50 \mathrm{~K}$ found by Samain et al. (1975) from the continuum around $158 \mathrm{~nm}$, and that of $4400 \mathrm{~K}$ near $160 \mathrm{~nm}$ by Parkinson and Reeves (1969). A kinetic temperature of $4500 \mathrm{~K}$ was obtained by de Jager (1963), who based his calculations on the early high solar irradiance measurements. Eddy et al. (1969) obtained $4370 \mathrm{~K} \pm 260 \mathrm{~K}$ from infrared observations in the band from $238 \mu \mathrm{m}$ to $312 \mu \mathrm{m}$. It should be noted that infrared CO bands indicate even temperatures below $4000 \mathrm{~K}$ in some regions of the solar atmosphere (Ayres and Testerman 1981). Most of the emission lines are produced above the temperature minimum and stem from the chromosphere, transition region or corona of the solar atmosphere.

2.1.1.2 The Spatial Structure Observations at high spatial resolution of the temperature minimum of the solar atmosphere in the VUV spectral range near $160 \mathrm{~nm}$ are available from the High Resolution Telescope and Spectrograph (HRTS) (Cook et al. 1983) and the Transition Region Camera (TRC) (Foing and Bonnet 1984). They not only clearly showed the chromospheric network lanes as an ensemble of discrete dots in brightness, but also many tiny bright points (with dimensions of $\approx 1 \mathrm{Mm}$ ), called grains, in the inter-network area, as can be seen from the TRACE image in Fig. 2.

Cook and Ewing (1990) found a linear relationship between these bright dots at $160 \mathrm{~nm}$ and the photospheric magnetic field strength. However, the heating represented by these features is not even sufficient to maintain the minimum temperature, and thus additional heating is required.

Although the magnetic field is not directly observable in the VUV, it is important to recall that the chromospheric network is some kind of projection of the magnetic structure of the supergranulation cells in the outer convection zone of the Sun. In this context, we have to mention even smaller magnetic structures of the lower photosphere that have been observed outside the VUV range. In the visible continuum near H I H $\alpha$, Dunn and Zirker (1973) saw 
the magnetic filigree, a string of grains in the network with typical sizes ${ }^{2}$ of $\approx 0.25^{\prime \prime}$, and magnetic field strengths from $10 \mathrm{mT}$ to $0.15 \mathrm{~T}$ (100 G to $1500 \mathrm{G}$ ). Berger et al. (1995) and Wiehr et al. (2004) found sub-arcsecond photospheric bright points in the "G band" at $430.5 \mathrm{~nm}$. The smallest measured diameter was near $100 \mathrm{~km}\left(0.14^{\prime \prime}\right.$ in angular extent).

\subsubsection{The Chromosphere}

This section reviews the thermal and spatial structures of the chromosphere, chromospheric oscillations, and high-resolution hydrogen Lyman lines and continuum. We also review whether the temperature increase with height is a permanent feature of the chromosphere, and whether the chromospheric network is a fundamental structure of the lower solar atmosphere with network lanes and inter-network areas. The dynamical behaviour of the quiet Sun is indicated by chromospheric oscillations, and its interpretation primarily due to the solar $p$ modes.

2.1.2.1 The Thermal Structure The chromosphere bridges the range from the temperature minimum of the solar atmosphere near $4500 \mathrm{~K}$ to the steep increase in the transition region above $2.5 \times 10^{4} \mathrm{~K}$. Limb spectra of the chromosphere in the range $200 \mathrm{~nm}$ to $320 \mathrm{~nm}$ were obtained from Skylab (Doschek et al. 1977). The average quiet-Sun temperature distribution was derived by Vernazza et al. (1981) from Skylab observations in the wavelength range from $49 \mathrm{~nm}$ to $140 \mathrm{~nm}$. They established that a decrease in the photosphere to the temperature minimum is followed by a slow increase in the chromosphere.

Also with Skylab data, Rosenberg et al. (1977) and Feldman and Doschek (1977a) studied the emission spectrum of the hydrogen Balmer series above the limb of a quiet region, a coronal hole and three active regions. The profiles of the series members 9 to 31 were observed in the near-ultraviolet range from $383.5 \mathrm{~nm}$ to $366.1 \mathrm{~nm}$. Electron densities were determined from the line broadening of the high members by the Stark effect. At a height of $2^{\prime \prime}$ above the limb, the electron density was $\approx 2 \times 10^{11} \mathrm{~cm}^{-3}$, independent of the region. By assuming that the spectra were emitted from a $7800 \mathrm{~K}$ plasma, nonthermal mass motions of $\approx 14 \mathrm{~km} \mathrm{~s}^{-1}$ were deduced.

The main topic of recent research is whether the temperature increase with height is a permanent feature of the chromosphere or only the result of an averaging process and induced by the observational methods (cf., Carlsson and Stein 1995; Kalkofen et al. 1999; Ayres 2002). Although this question is intimately related to the dynamics that will be treated in Sect. 2.1.2.3, several findings should be mentioned here as they might shed some light on the situation: (a) The Si I ionization continuum is conspicuously limb brightened below $152 \mathrm{~nm}$, whereas the continuum radiance does not change towards the limb above this wavelength (Tousey 1964); (b) Athay and Dere (1990) using data of HRTS, established that C I and $\mathrm{O}$ I lines are seen in emission in inter-network regions without gaps, on a scale of slightly less than 1"; and (c) all spectral lines with wavelengths below $160 \mathrm{~nm}$, even if emitted by atoms and singly charged ions, are observed (Carlsson et al. 1997; Judge et al. 1997) only in emission, both in inter-network and network regions, by the Solar Ultraviolet Measurements of Emitted Radiation (SUMER) spectrometer (Wilhelm et al. 1995) on the Solar and Heliospheric Observatory (SOHO).

\footnotetext{
${ }^{2}$ Distances on the Sun are often given in the literature in angular units with $1^{\prime \prime} \approx 720 \mathrm{~km}$ from 1 ua $=$ $1.49597870691(6) \times 10^{11} \mathrm{~m}$ (BIMP 2006).
} 
2.1.2.2 The Spatial Structure The chromospheric network is prominently seen, for instance, in the Ca II K line at $393.4 \mathrm{~nm}$ formed between about $5000 \mathrm{~K}$ and $8000 \mathrm{~K}$ (Vernazza et al. 1981). The network covers the entire quiet Sun and the coronal holes, and has typical cell dimensions of $20 \mathrm{Mm}$. It is related to the supergranulation structure of the outer convection zone. The network is also visible in chromospheric VUV lines, such as the hydrogen Lyman series. As an example, we can refer to Fig. 10 in Part I showing the full Sun in H I Ly $\alpha$ (obtained by the Multi-Spectral Solar Telescope Array, MSSTA). In addition to the quiet-Sun network, some active regions are also visible in that image. Higher-resolution Ly $\alpha$ observations were obtained by Basri et al. (1979) on the first rocket flight of HRTS. Ly $\alpha$ filtergrams of the TRC showed small-scale features at the resolution limit of the instrument of less than 1". These and other observations by Bonnet et al. (1980), Foing and Bonnet (1984), and Damé et al. (1986) demonstrated the inhomogeneous and filamentary structure of the solar atmosphere.

It should be mentioned that the generation process of the $\mathrm{H} \mathrm{I} \mathrm{Ly} \alpha$ line in the solar atmosphere is very complex, and involves not only the chromosphere, but also the transition region and even the corona (cf., Vourlidas et al. 2001). Similar complications exist for the higher members of the H I Lyman series and the continuum. Some of these aspects are discussed in Sect. 2.1.2.4.

Very small transition-region structures (less than $1^{\prime \prime}$ in angular extent) were suggested by Doschek et al. (2004) as an explanation of the features found in their correlation studies of chromospheric and transition-region emission lines. Wilhelm and Kalkofen (2003) obtained a correlation between the spatial radiance variations in chromospheric and coronal lines, which is more significant than the corresponding correlation of transition-region with coronal lines, a result that also indicates very small transition-region structures.

Figure 3 is an image of the Sun in H I Ly $\epsilon$ of the SUMER atlas (Feldman et al. 2003) with reduced spatial resolution. In quiet-Sun regions, including coronal holes, the chromospheric network apparently is the fundamental structure of the lower solar atmosphere with network lanes and inter-network areas. Some active regions near the centre of the disk can also be seen.

Filaments, prominences and smaller structures (e.g., spicules) also contain chromospheric material, but reach into the corona. They will be discussed under separate headings in Sects. 2.2.1 and 2.2.2.

\subsubsection{Chromospheric Oscillations Oscillations in the chromosphere are indications of} the dynamical behaviour of the quiet Sun. First discovered as oscillations with a period of $300 \mathrm{~s}$ in the photosphere and chromosphere (Leighton et al. 1962), they subsequently were also seen in VUV emission lines, both in radiance and velocity (Chapman et al. 1972; Chipman et al. 1976; Chipman 1977; Martic et al. 1991; Hoekzema et al. 1997; Carlsson et al. 1997; Curdt and Heinzel 1998; Hansteen et al. 2000; McIntosh et al. 2001; Krijger et al. 2001). Judge et al. (2001) studied oscillations in the chromosphere using SOHO and the Transition Region and Coronal Explorer (TRACE) (Handy et al. 1999) continuum observations in the $170 \mathrm{~nm}$ band. They discovered "magnetic shadows" devoid of oscillations near network lanes and found that the chromosphere oscillates primarily in response to the solar $p$ modes, in agreement with Ultraviolet Spectrometer and Polarimeter (UVSP) observations during the Solar Maximum Mission (SMM) that showed evanescent waves are driven by photospheric oscillations (Doyle et al. 1997). The magnetic shadows were further investigated by McIntosh and Judge (2001). Banerjee et al. (2001a) saw network oscillations with coherence times of $10 \mathrm{~min}$ to $20 \mathrm{~min}$. The power spectrum of the radiance and Doppler velocity showed activity near $3 \mathrm{mHz}$ in chromospheric and transition-region lines, but not in the coronal data of the line $\mathrm{Mg}$ X $62.5 \mathrm{~nm}$. 
Fig. 3 H I Ly $\epsilon$ image of the Sun obtained by SUMER on 12 May 1996 (from Feldman et al. 2003). The chromospheric network structure of the quiet Sun and of active regions near the centre of the disk are the most prominent features in this image

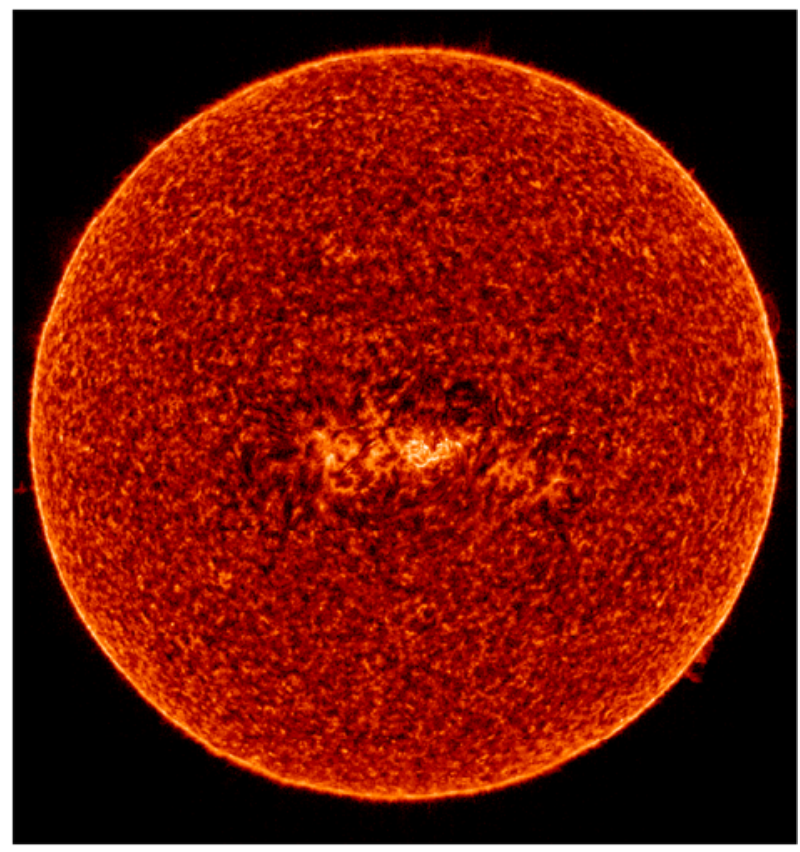

Fleck and Deubner (1989) postulated a "magic height" between $880 \mathrm{~km}$ and $1200 \mathrm{~km}$ at which the oscillatory behaviour of the chromosphere changed dramatically. Studies of the H I Lyman continuum (Wilhelm and Kalkofen 2003) and the C II $133.5 \mathrm{~nm}$ multiplet (Judge et al. 2003) both indicated a heating of the upper chromosphere by magnetic effects and not by acoustic waves. However, Gallagher et al. (1999) found indications of heating by acoustic shocks even in the transition region near $2.5 \times 10^{5} \mathrm{~K}$. Evidence for atmospheric gravity waves was found from TRACE observations (Rutten and Krijger 2003).

\subsubsection{The Lyman Lines of Hydrogen and the Continuum The H I Lyman continuum} is generated in the chromosphere by radiative recombination of ionized hydrogen into the ground state. The ionization (as well as excitation) can occur by collisional or radiative processes. High-resolution spectra of the Lyman continuum from $67.1 \mathrm{~nm}$ to the edge at $91.2 \mathrm{~nm}$ have been obtained by SUMER yielding kinetic electron temperatures of $T_{\mathrm{e}}^{\mathrm{N}} \approx 9 \times 10^{3} \mathrm{~K}$ over the chromospheric network and $T_{\mathrm{e}}^{\mathrm{I}} \approx 8 \times 10^{3} \mathrm{~K}$ in inter-network regions from considerations of the spectral slopes. The corresponding radiances, however, are much lower than those expected from these temperatures, and indicate departures from a local thermodynamic equilibrium (Wilhelm and Kalkofen 2003).

The H I Lyman lines are emitted by transitions within the excited hydrogen atoms to the ground state (cf., Part I, Sect. 9.1). A spectrum showing the H I Lyman series members with $6 \leq n_{2} \leq 21$ and the Lyman continuum edge is plotted in Fig. 19 of Part I for a solar prominence. All lines appear to be of Gaussian shape (cf., however, Sect. 2.2.2.1). The lower solar atmosphere is optically thick for most of the H I Lyman lines. A detailed study of the Lyman series (except $\mathrm{Ly} \alpha$ ) in quiet-Sun areas on the disk was performed by Warren et al. (1998). Effects of radiative transfer are apparent at least up to $\operatorname{Ly} \lambda$, and the lines $\operatorname{Ly} \beta$ to Ly $\epsilon$ are selfreversed, because the line centres are absorbed at higher altitudes. The strongly self-reversed $\operatorname{Ly} \alpha$ and $\operatorname{Ly} \beta$ lines and their variations during solar cycle 23 have been studied by Lemaire 


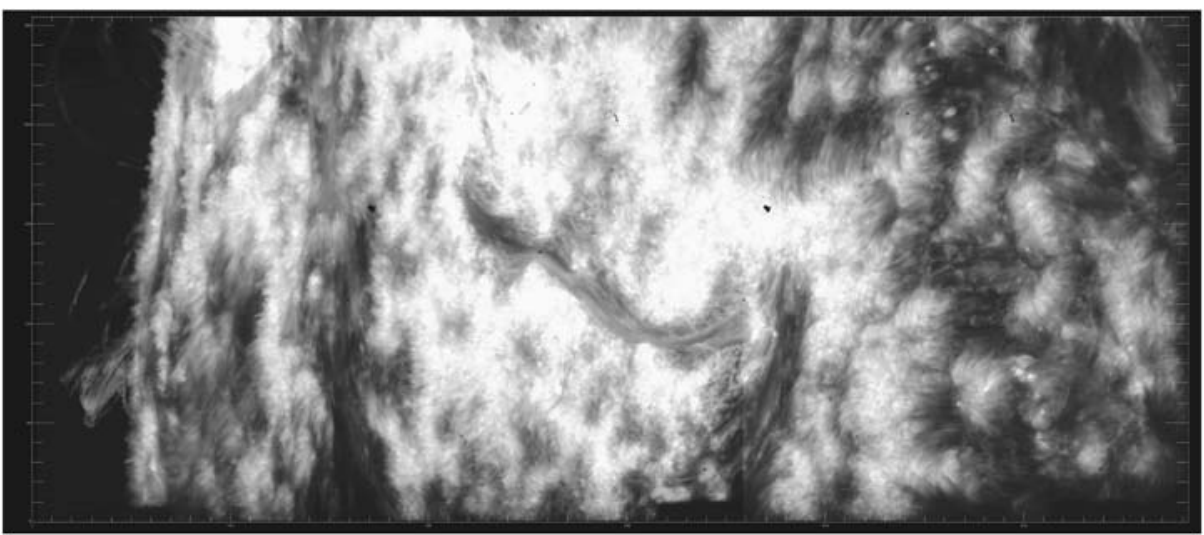

Fig. 4 H I Ly $\alpha$ image of the western solar limb, with active regions (AR 9997, 9998, and 9999) and quiet-Sun areas obtained by VAULT aboard a sounding rocket launched on 14 June 2002. The size of the image is $580^{\prime \prime} \times 250^{\prime \prime}$ (Courtesy C.M. Korendyke and A. Vourlidas)

et al. (1998, 2005) using the scattered full-disk radiation in the SUMER telescope and calibrating it with the help of the Solar-Stellar Irradiance Comparison Experiment (SOLSTICE) measurements (Woods et al. 2000). For the interaction of the cool hydrogen in our planetary system with the solar Ly $\alpha$ radiation, the spectral irradiance at the line centre is of particular importance. In order to derive this value from Ly $\alpha$ line irradiance measurements, Emerich et al. (2005) inferred a new relationship between the central spectral irradiance and the total irradiance of Ly $\alpha$ from SUMER observations as a revision of an older semi-empirical relation obtained from Orbiting Solar Observatory (OSO-5) data (Vidal-Madjar 1975).

Images in the $\operatorname{Ly} \alpha$ line of the solar atmosphere at the highest spatial resolution have been recorded to date by the Very high Angular-resolution Ultraviolet Telescope (VAULT) (Korendyke et al. 2001). An example from the second flight of the VAULT payload is shown in Fig. 4.

\subsubsection{The Transition Region}

The structure of the transition region is still an unsolved problem despite a tremendous amount of VUV data available in the last decades. We review the dependence of the nonthermal line widths of transition-region lines on their formation temperatures under varying plasma conditions. We also review the helium VUV line emissions, concluding that the physics of its VUV spectrum is still an open question.

2.1.3.1 The Thermal Structure Brueckner and Moe (1972) observed spectral lines (O III to O VI and others) in the wavelength range from $140 \mathrm{~nm}$ to $179 \mathrm{~nm}$ and found line widths in excess of the thermal Doppler broadening that is expected from the electron temperature at the maximum of the ionic fractions. Line widths with nonthermal broadenings from $10 \mathrm{~km} \mathrm{~s}^{-1}$ to $24 \mathrm{~km} \mathrm{~s}^{-1}$ were also seen for Si II, C II, Si III, and C IV lines on a rocket flight by Boland et al. $(1973,1975)$. Observations by OSO 8 provided further evidence that the transition-region line Si IV at $139.3 \mathrm{~nm}$ is much wider than deduced from its formation temperature (Francis and Roussel-Dupré 1977). The dependence of the nonthermal line width of transition-region lines on the formation temperature was studied with Skylab S082B limb data by Doschek et al. (1976a) for quiet-Sun conditions and Feldman et al. (1976a) for a 


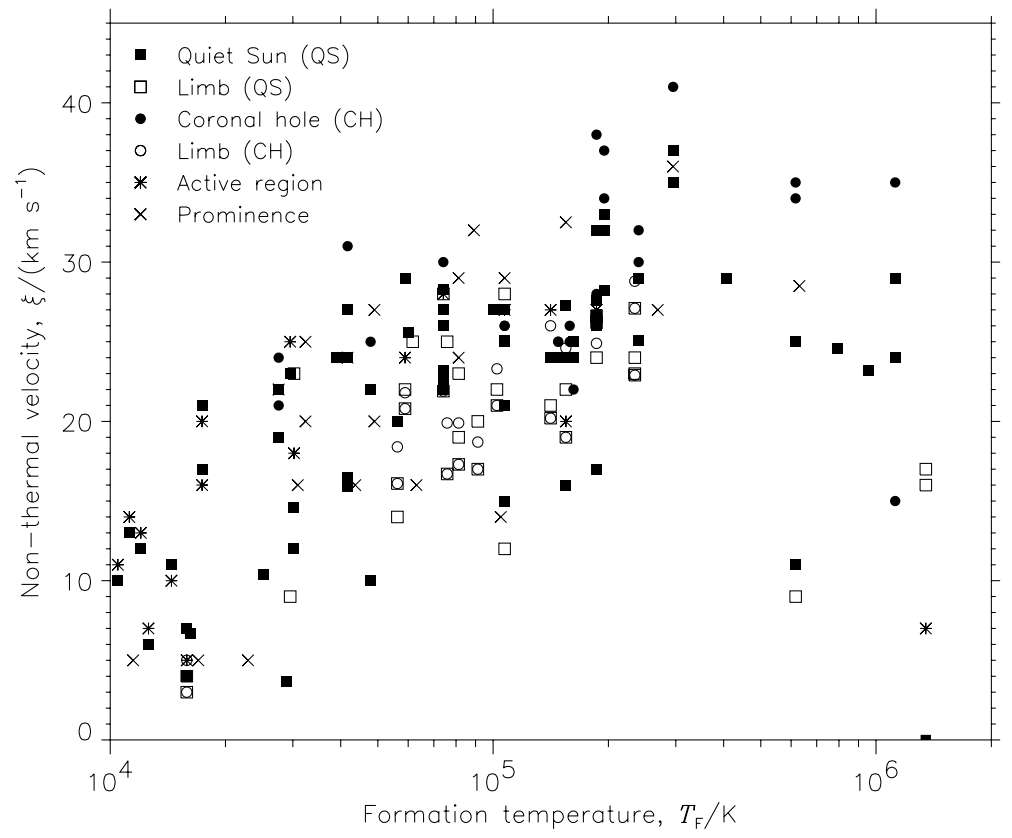

Fig. 5 Nonthermal ion velocities (turbulent speeds) as derived from spectral line widths as a function of the formation temperature observed on the disk by several instruments under widely varying plasma conditions in the solar atmosphere from the chromosphere through the transition region to the corona (data from Boland et al. 1973; Doschek et al. 1976a; Feldman et al. 1976a; Kjeldseth Moe and Nicolas 1977; Dere and Mason 1993; Chae et al. 1998a; de Boer et al. 1998; Peter 1999a; Xia 2003; Cirigliano et al. 2004; Akiyama et al. 2005)

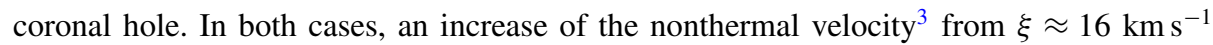
at $3 \times 10^{4} \mathrm{~K}$ to $\approx 28 \mathrm{~km} \mathrm{~s}^{-1}$ at $3 \times 10^{5} \mathrm{~K}$ was observed in agreement with the results of Boland et al. (1975). From SUMER observations, Chae et al. (1998a) found a maximum of $30 \mathrm{~km} \mathrm{~s}^{-1}$ at approximately $2.5 \times 10^{5} \mathrm{~K}$, under the assumption that the ion temperature was not different from the formation temperatures, $T_{\mathrm{F}}$.

In Fig. 5 we have compiled many data points derived from measurements by various authors in order to compare the results and show the agreement of the observations within the natural variability of the Sun. It is remarkable that there are no systematic differences in $\xi$ for the various solar conditions. From values of $\xi \approx 10 \mathrm{~km} \mathrm{~s}^{-1}$ in the chromosphere, the turbulence increases by more than $10 \mathrm{~km} \mathrm{~s}^{-1}$ in the lower transition region $\left(<2 \times 10^{5} \mathrm{~K}\right)$. It appears as if this compilation indicates a second increase of $\approx 10 \mathrm{~km} \mathrm{~s}^{-1}$ at $2 \times 10^{5} \mathrm{~K}$. It might be related to the broad line component found and discussed by Peter $(2000,2001)$. At coronal temperatures, there is a tendency of decreasing $\xi$, but the data spread is very large (cf., Sects. 3.3.2 and 3.6.3).

For the C IV line at $154.8 \mathrm{~nm}$, with a formation temperature of $1 \times 10^{5} \mathrm{~K}$, Peter (1999a) obtained a correlation of the radiance of lines in quiet-Sun regions with both the Doppler shift towards red and the line broadening. This correlation was not observed in coronal holes. Dammasch et al. (1999a) found a positive correlation between the radiance and the

\footnotetext{
${ }^{3}$ For a discussion of the line broadening and its relation to the ion temperature and the nonthermal turbulent contribution, $\xi$, see, e.g., Mariska (1992) or Part I, Sect. 3.5.
} 
width of this line, and a similar correlation for the Si II line. Such a relationship was also observed by Lemaire et al. (1999) and Wilhelm (2000a) for several lines in quiet-Sun areas and coronal holes. The centre-to-limb variations of the width of many lines observed by Dammasch et al. (1999b) suggest a significant amount of horizonal motions in the transition region. The centre-to-limb variations observed by Doyle et al. (2000) for C IV and He I at $58.4 \mathrm{~nm}$ emitted from optically thick plasma regions are explained as opacity effects, and the increase in the line width at $\approx 10 \mathrm{Mm}$ above the limb is interpreted as being due to contributions from unresolved spicules and macrospicules (Doyle et al. 2005). Finally, the widths of many lines are positively correlated with their formation temperatures (Dammasch et al. 1999b).

First indications that spectral line profiles could only be fitted by more than one Gaussian curve were found with OSO 8 (Shine et al. 1976). These profiles were also recognized by Kjeldseth Moe and Nicolas (1977) in Skylab observations and studied further by Dere and Mason (1993) with HRTS data. Chae et al. (1998a) obtained a two-Gaussian fit with a Doppler width of $22 \mathrm{~km} \mathrm{~s}^{-1}$ and $55 \mathrm{~km} \mathrm{~s}^{-1}$ for the $\mathrm{S}$ VI line at $93.3 \mathrm{~nm}$. In another study using SUMER observations, the wings of transition-region lines have been modelled by two Gaussian distributions that are offset from a narrow central profile and interpreted as bidirectional motions caused by explosive events (Wilhelm 2000b) (cf., Sect. 2.2.1.3). These events are ubiquitous on the disk under all solar conditions.

Such broad wings have also been observed for strong emission lines of $\alpha$ Cen A (cf., Wood et al. 1997; Pagano et al. 2004). Redfield et al. (2002) concluded that the broad component found in star observations can be considered as evidence for nonthermal motions related to magnetic reconnection and explosive events. Peter (2001), however, suggested that the wide component is representative of a low-density population in solar network funnels (cf., Sect. 3.3.2). Narrow and wide profiles of transition-region lines have also been found in the lanes of the chromospheric network, whereas the narrow component stems from the network loops. Studying a composite full-Sun C IV line profile, Peter (2006) concluded that its broad component is not caused by unresolved small-scale events, but rather by a relationship of the radiance with the Doppler shift of the emission line in the chromospheric network.

Marsch et al. (1999, 2000) observed lines of the H I Lyman series above the limb in the altitude from $12 \mathrm{Mm}$ to $18 \mathrm{Mm}$ and obtained increasing hydrogen temperatures from $1 \times$ $10^{5} \mathrm{~K}$ to $2 \times 10^{5} \mathrm{~K}$, which are thought to be also representative for the proton temperatures, due to charge-exchange processes (cf., Sect. 2.1.4.1). The temperature gradient is relatively small and might be influenced by spatial inhomogeneities (cf., Sect. 2.1.2.2).

In addition to the ion temperatures discussed so far, the electron temperature is of interest. In general, as mentioned earlier, it is assumed that $T_{\mathrm{i}}=T_{\mathrm{e}}$, but direct measurements are important to check this. Feldman and Laming (1994) did not find any relationship between the properties of plasmas with temperatures of $T_{\mathrm{e}} \leq 7 \times 10^{5} \mathrm{~K}$ and those with $T_{\mathrm{e}} \geq 1 \mathrm{MK}$ (cf., also Reeves et al. 1974; Reeves 1976; Wilhelm and Kalkofen 2003). Filter and double-filter ratios of TRACE have been studied by Chae et al. (2002), who observed transition-region loops with $T_{\mathrm{e}}=2.4 \times 10^{5} \mathrm{~K}$ as well as coronal loops with much higher temperatures along the same LOS (see also Sect. 2.1.4.4). Spectroscopic line ratios obtained by the Coronal Diagnostic Spectrometer (CDS, Harrison et al. 1995, 1997) or SUMER confirm the relatively low temperatures in transition-region loops (Brekke 1999; Chae et al. 2000a). The major fraction of the extreme-ultraviolet (EUV) emission in the $8 \times 10^{4} \mathrm{~K}$ to $8 \times 10^{5} \mathrm{~K}$ range, i.e., at transition-region temperatures, stems from small cool loops and other unresolved structures that do not reach coronal temperatures at all (Fludra et al. 1997). 
2.1.3.2 The Helium Emissions The helium spectra He I and He II in the VUV range have many characteristics that place them apart from other transition-region emissions. A special section might therefore be in order to discuss the relevant data and the results obtained.

The generation of the EUV helium spectrum is still one of the problems that have not found a clear answer. Zirin (1988), in a review on this topic, concluded that the available evidence favours the photoionization-recombination mechanism, i.e., a dependence upon the coronal EUV radiation. Collisional excitation is another generation process that was discussed by Athay (1988). The line radiances within the He I Balmer multiplet at $160.4 \mathrm{~nm}$ recorded above the limb in a number of regions were studied by Feldman et al. (1975) and Seely and Feldman (1985). It was found that collisional excitation from the 1s ground state is the main population mechanism of the $3 \mathrm{~s}, 3 \mathrm{p}$ and $3 \mathrm{~d}$ upper levels at angular distances of $4^{\prime \prime}$ to $8^{\prime \prime}$ above quiet regions. In a solar spectrum recorded by a grazing-incidence spectrometer (GIS) on a sounding rocket, Feldman and Behring (1974) and Doschek et al. (1974) measured profiles of the He I $58.4 \mathrm{~nm}$ and He II $30.4 \mathrm{~nm}$ lines and found them both to be of approximately Gaussian shape.

Other ideas are based on the "velocity filtration mechanism" of nonthermal electrons (Scudder 1992) or on the "velocity redistribution" (Jordan 1975). Andretta et al. (2000) further studied the latter effect with the Solar Extreme-ultraviolet Research Telescope and Spectrograph (SERTS), and confirmed that the He II $30.4 \mathrm{~nm}$ line profile is nearly of Gaussian shape both in the quiet Sun and in active regions. A Gaussian shape is also characteristic of the He I $58.4 \mathrm{~nm}$ line in the quiet Sun, but self-reversals are found in some regions of coronal holes and above filaments, but not in prominences (Wilhelm et al. 2005b). These authors also discussed unidentified broad emission features on either side of the helium line in superimposed first- and second-order spectra of SUMER, which have subsequently been identified as autoionizing transitions of neutral sulfur (Avrett et al. 2006).

Skylab observations revealed that the helium lines have anomalous radiances in comparison with other transition-region lines (cf., Macpherson and Jordan 1999).

CDS observations in the helium lines He I $58.4 \mathrm{~nm}, 53.7 \mathrm{~nm}$ and He II at $30.4 \mathrm{~nm}$ (and other EUV lines) in combination with synoptic observations by the Extreme-ultraviolet Imaging Telescope (EIT; Delaboudinière et al. 1995) led Andretta et al. (2003) to the conclusion that the photoionization-recombination mechanism alone cannot explain the formation of He II $30.4 \mathrm{~nm}$. Andretta and Jones (1997), on theoretical grounds, favour a two-layer formation model of the helium spectrum with mixed contributions of the excitation processes. Ravindra and Venkatakrishnan (2003), using EIT observations, found that the He II $30.4 \mathrm{~nm}$ network brightenings are independent of coronal EUV radiation, but are strongly correlated with the photospheric magnetic field strength as measured by the Kitt Peak National Solar Observatory (KPNSO) and the Michelson Doppler Imager (MDI; Scherrer et al. 1995) on SOHO. It thus appears as if the details of the formation of the He I spectrum are still an open question. Studies by Fredvik and Maltby (1999) in sunspot regions using CDS also led to inconclusive results.

The solar atmosphere is optically thick for both He I $58.4 \mathrm{~nm}$ and He II $30.4 \mathrm{~nm}$. Gallagher et al. (1998) arrived at optical depths of $\tau \approx 1 \times 10^{4}$ for these lines. An enhanced line width and blue shifts of the He I $58.4 \mathrm{~nm}$ line were found in coronal holes by Peter (1999b). The relation of the radiance with the line shift indicates that optical depth effects might be the cause of these findings and not any plasma flows. It should be noted that the radiance is less in coronal holes than in quiet-Sun regions (cf., Fig. A.40 of Feldman et al. 2003).

The Solar Extreme-ultraviolet Monitor (SEM) on SOHO (Hovestadt et al. 1995; Ogawa et al. 1998; McMullin et al. 2004) has provided solar irradiance data of the He II line at $30.4 \mathrm{~nm}$, which are useful not only for solar studies but also interstellar and interplanetary 
Fig. 6 The quiet Sun seen in the transition-region line $\mathrm{C}$ IV at $154.8 \mathrm{~nm}$ formed at $1 \times 10^{5} \mathrm{~K}$ on 4 February 1996 (from Wilhelm 1999)

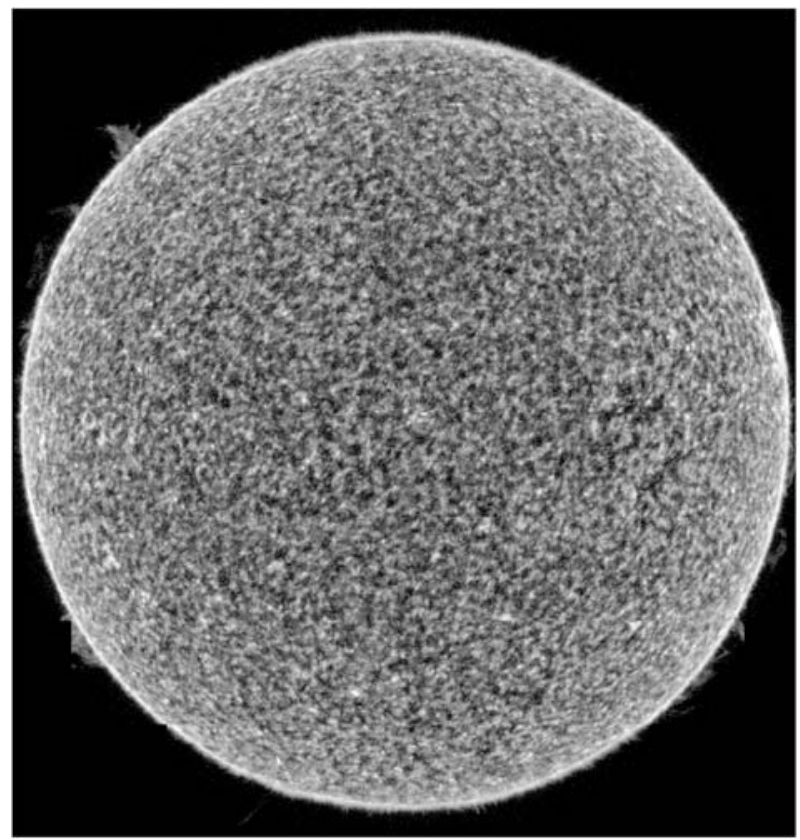

investigations of helium and its interaction with the solar radiation (Lallement et al. 2004a, 2004b). An interesting result obtained by Auchère et al. (2005) from EIT He II 30.4 nm observations is the heliospheric flux distribution in this line over a large interval of the solar cycle 23 .

2.1.3.3 The Spatial Structure The chromospheric network structure (cf., Sect. 2.1.2.2) is also prominently seen in all transition-region lines formed between $2.5 \times 10^{4} \mathrm{~K}$ and $\approx 4 \times 10^{5} \mathrm{~K}$. It fades out in emission at coronal temperatures above $6 \times 10^{5} \mathrm{~K}$. First multiwavelength observations in the VUV reported by Reeves et al. (1974), Reeves (1976) and Feldman et al. (1976a) showed that the contrast between network lanes and cell interiors had a maximum at lines emitted between $1 \times 10^{5} \mathrm{~K}$ and $2 \times 10^{5} \mathrm{~K}$.

A two-dimensional model of the chromosphere, transition region, and corona by Gabriel (1976) was able to describe the early observations. This model placed the primary transition region on expanding magnetic flux tubes above the network lanes and a thin secondary transition region above the cells.

Huber et al. (1974) and Bohlin and Sheeley (1978) showed that the network seen on the disk is remarkably similar in quiet-Sun areas and coronal holes but, as seen on the limb, has a greater vertical extension in holes. This can be noticed from a full-Sun image in the CIV line at $154.8 \mathrm{~nm}$ in Fig. 6 obtained by SUMER on 4 February 1996, when extended polar coronal holes were present during the sunspot minimum (cf., Fig. 7 showing the coronal holes observed by EIT in Fe XII on the same day). This behaviour of the transition-region emission has been explained with MDI magnetograms and magnetic-field extrapolations as being caused by the difference in the loop geometry: whereas loops are present in both regions, the loops in coronal holes are generally lower than those in quiet regions, and the high loops are entirely lacking in coronal holes (Wiegelmann and Solanki 2004). Similar conclusions were reached in the paper by Marsch et al. (2006) on the magnetic structure of the solar transition region as observed in various ultraviolet lines emitted at different temperatures. 
Fig. 7 The corona of the Sun seen on 4 February 1996, during the last sunspot minimum in the EIT bandpass containing Fe XII lines near $19.5 \mathrm{~nm}$ (courtesy of F. Auchère and the EIT consortium)

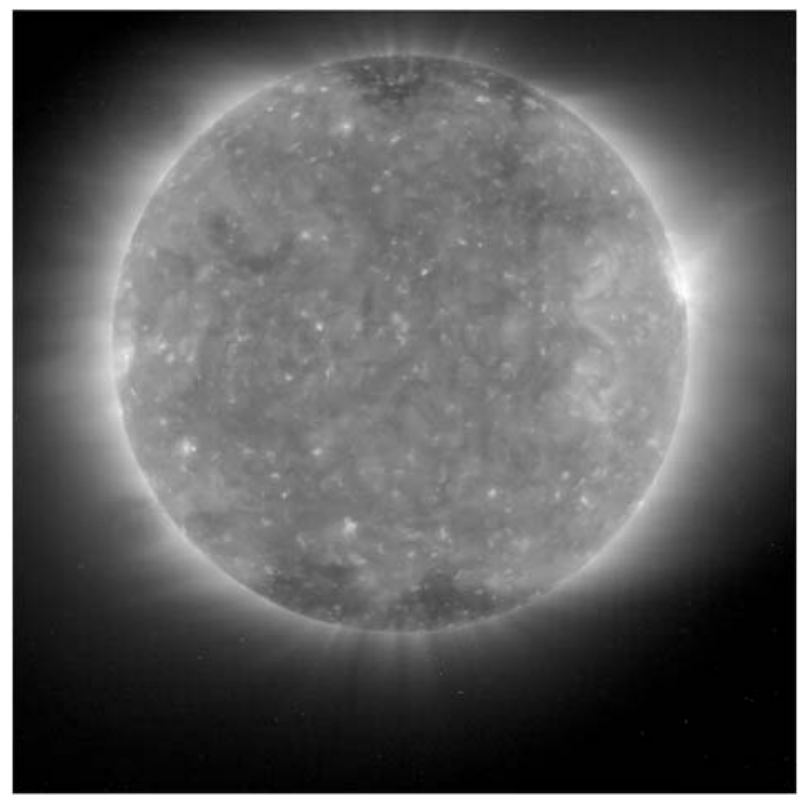

Doschek et al. (1975a) also found evidence for an extended transition region in coronal holes as compared to quiet-Sun regions. Detailed limb-brightening curves of many transition-region lines above a coronal hole were derived from S082B spectrograph observations (Feldman et al. 1976a). Profiles of emission lines near the limb were also obtained by Mariska et al. (1978) and compared with quiet-Sun height profiles. No difference was found for atomic lines, but high-temperature emissions were enhanced in quiet-Sun regions near the maximum of the limb brightening at $\approx 4^{\prime \prime}$ above limb. Since the extension of the emission was greater above coronal holes (Doschek et al. 1976a), the ratio $L_{\mathrm{QS}} / L_{\mathrm{CH}}$ was smaller than one at higher altitudes. Without adequate spatial resolution to identify the network, OSO-4 observations had indicated before that the radiances of transition-region lines and continua did not decrease significantly in coronal holes (Munro and Withbroe 1972). A very strong limb brightening of the S VI line at $93.3 \mathrm{~nm}$ (a factor of $\approx 10$ ) was observed by Lemaire et al. (1997).

The structure of the transition region is still a matter of debate. Feldman (1983) suggested that most of the transition-region radiation stems from unresolved fine structures, which are not part of a continuous chromosphere-corona interface, and Neupert et al. (1992) concluded that the traditional transition region is only responsible for a small part of the EUV emission.

The width of the average network lane has a minimum of about $6^{\prime \prime}$ near $2 \times 10^{5} \mathrm{~K}$, represented by O IV and $\mathrm{N} v$ lines. The O IV line diagnostics indicated an electron density of $\approx 1 \times 10^{9} \mathrm{~cm}^{-3}$ here. Under the assumption of ionization equilibrium, Doschek et al. (1978) derived electron densities at transition-region temperatures from O III, C III, and Si IV line ratios (observed with the S082B spectrograph) and found values of $\approx 2 \times 10^{10} \mathrm{~cm}^{-3}$. For lines formed at temperatures below $1 \times 10^{5} \mathrm{~K}$ (O I, C II, He I, and He II), the width of the lanes increases to $\approx 10^{\prime \prime}$ (Feldman et al. 1976b; Gallagher et al. 1998). As can be seen from Fig. 8, there is another, steeper increase at coronal formation temperatures. From $\mathrm{O} v$ and Si III line-ratio studies with SUMER, Doschek et al. (1998a) found that the electron pressure is nearly constant in the low transition region. 


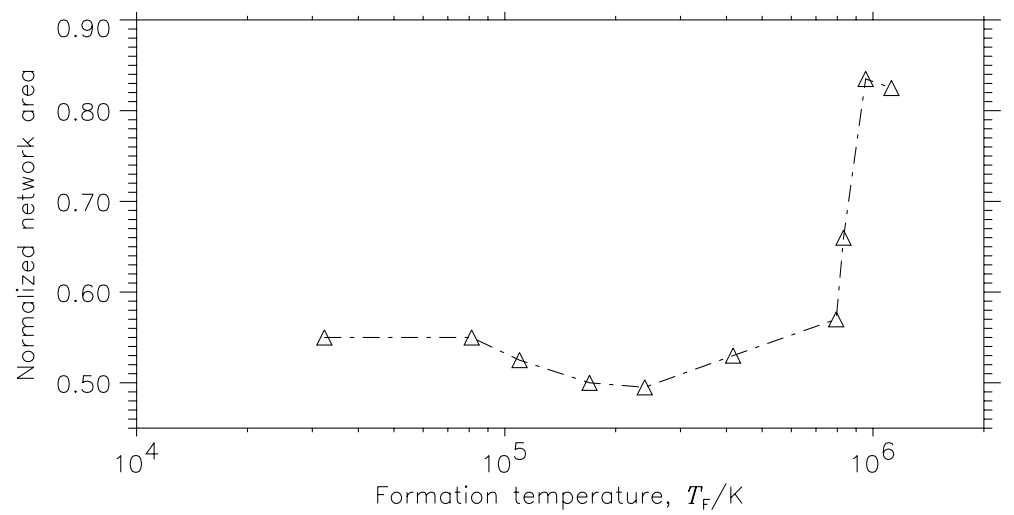

Fig. 8 The normalized network area seen in various emission lines as a function of the line formation temperature, $T_{\mathrm{F}}$ (after Gallagher et al. 1998)

Patsourakos et al. (1999) showed with CDS observations that the network widening with temperature is fully consistent with the Gabriel model (Gabriel 1976). Landi et al. (2000) later determined an electron density $n_{\mathrm{e}} \approx 1 \times 10^{10} \mathrm{~cm}^{-3}$ in the loop-like fine structures of the network. Network bright points have been found to coincide with magnetic neutral lines, and are often also seen as enhanced coronal structures (Falconer et al. 1998). ChiuderiDrago et al. (1977), and recently Marsch et al. (2006), concluded from UV and radio observations that hot and cold plasmas must coexist in coronal holes at low altitudes. The same conclusion was reached for quiet-Sun regions near the equator from VUV observations of lines with different formation temperatures (Wilhelm et al. 2002a). A review by Feldman et al. (2000a) summarizes recent observations and interpretations of the morphology of the solar atmosphere during the sunspot minimum. The strong C III $97.7 \mathrm{~nm}$ line, formed at $8.3 \times 10^{4} \mathrm{~K}$, shows the network structure with the best contrast and resolution (cf., Fig. 13 in Sect. 3.1). Dowdy et al. (1986) introduced a model with funnels and a hierarchy of magnetic loops in and across the network lanes that accounts for most of the VUV emission.

The occurrence probabilities of the network and inter-network radiance variations of transition-region lines are, if plotted on a logarithmic scale, normally distributed (Lemaire et al. 1997; Wilhelm et al. 1998a; Griffiths et al. 1999; Kretzschmar et al. 2004). The widths of the distribution functions vary with the formation temperature and have a maximum near $1 \times 10^{5} \mathrm{~K}$. Dammasch et al. (1999c) found in addition that the shift and width distributions of the Si II, C IV and Ne VIII lines are also of Gaussian shape. Dere et al. $(1984,1987)$ deduced a sub-resolution structure of the transition region from HRTS observations on Spacelab 2 $\left(\approx 1^{\prime \prime}\right.$ angular resolution) with a filamentary configuration and a low filling factor. A recent statistical study of full-Sun images in S VI and H I Ly $\beta$ lines by Buchlin et al. (2006) indicated the existence of small-scale structures in the transition region created by turbulence and intermittency.

2.1.3.4 Temporal Radiance Variations Nonperiodic variations of the radiance of transitionregion lines up to a factor of 1.5 on time scales of 1 min were seen with the Harvard College Observatory (HCO) instrument aboard Skylab (Vernazza et al. 1975). Later observations showed several other types of variations, which are discussed in Sect. 2.2. 
2.1.3.5 Plasma Flows An important review on mass flow and energy transport in the solar chromosphere and corona was published by Withbroe and Noyes (1977). It is therefore not necessary here to detail the early development in this field.

An average red shift of most of the transition-region lines ( $\mathrm{Si}$ II, C IV, O IV, and N V) was observed in many quiet-Sun disk spectra from the S082B spectrograph by Doschek et al. (1976b). Maximum shifts implied downflows of $15 \mathrm{~km} \mathrm{~s}^{-1}$. They were observed in network boundaries. Should the average shifts indicate real downflows, then the corona could support these for only a few minutes unless there is some upflow at other temperatures. No shifts in cell interiors were found, although the statistics were poor in this respect. Pneuman and Kopp (1978) presented evidence from the first HRTS flight in 1975 (Brueckner and Bartoe 1983) for downflows at the cell boundaries, in particular in the CIV $154.8 \mathrm{~nm}$ line (see Sect. 2.1.3.3). They suggested that this downflow is related to the return flux of spicular material (cf., Sect. 2.2.1.1), which provides about 100 times more mass than the solar wind carries away.

Using UVSP Dopplergram observations of C II, Si IV and C IV spectral lines made with a $3^{\prime \prime} \times 3^{\prime \prime}$ entrance slit, Gebbie et al. (1981) found larger downflows in brighter regions of the quiet-Sun network. Lacking a wavelength calibration, the absolute speed of the flows could not be determined, however, a bimodal behaviour of the LOS velocities as a function of the line radiance of C IV $154.8 \mathrm{~nm}$ emerged with a "network" branch and one with lower velocities in distinct bright points. A similar behaviour was also observed by Athay et al. (1983). Dere et al. (1989a) used HRTS C IV observations in coronal holes and quiet-Sun regions to demonstrate that this line is Doppler shifted towards longer wavelengths both under coronal-hole and quiet-Sun conditions, but with greater proportions of outflowing plasma in coronal holes.

With data obtained by an EUV coronal spectrometer on the Laboratory for Atmospheric and Space Physics (LASP) sounding rocket in 1987, Hassler et al. (1991) extended the investigations to shorter wavelengths and found no shift for the Ne VIII $77 \mathrm{~nm}$ line (albeit with a large uncertainty margin), while confirming the previous results at lower formation temperatures. Brekke (1993) studied the O v $137.1 \mathrm{~nm}$ line in HRTS spectra and found net average downflows of $8 \mathrm{~km} \mathrm{~s}^{-1}$. Doschek et al. (1976b) had reported no significant shift for the $\mathrm{O} v$ line at $121.8 \mathrm{~nm}$ formed at $2.4 \times 10^{5} \mathrm{~K}$ in the Skylab data. In contrast to this result, Brekke (1993) found a net redshift in the two O V lines at $121.8 \mathrm{~nm}$ and $137.1 \mathrm{~nm}$ in the data obtained by the HRTS instrument. He attributed the conflicting results for the O V $121.8 \mathrm{~nm}$ line to uncertainties in the laboratory wavelength of this line.

A review of red-shift observations by Brekke (1994) substantiated the average downflow hypothesis at transition-region temperatures, but indicated the need for further studies in order to resolve the discrepancies in the high-temperature regime. Brekke et al. (1997a), Warren et al. (1997a), and Chae et al. (1998b) continued the investigation of the average red shift with SUMER data. These studies confirmed a maximum red shift corresponding to more than $10 \mathrm{~km} \mathrm{~s}^{-1}$ near $2 \times 10^{5} \mathrm{~K}$ and provided evidence that downflows of about $5 \mathrm{~km} \mathrm{~s}^{-1}$ were present for Ne VIII at $77.0 \mathrm{~nm}$ and $\mathrm{Mg} \mathrm{X}$ at $62.5 \mathrm{~nm}$, assuming the standard laboratory wavelengths. Peter and Judge (1999) and Dammasch et al. (1999a, 1999d), on the other hand, came to the conclusion that the high-temperature lines are shifted towards blue in quiet-Sun areas (indicating outflows), and that the rest wavelengths of these lines have to be revised (cf., Sect. 9.1 of Part I). A compilation of many of the average LOS velocity measurements is shown in Fig. 9.

The temperature of the average red-shift maximum just above $1 \times 10^{5} \mathrm{~K}$ coincides with that of the maximum of the contrast between network and inter-network areas (cf., Sect. 2.1.3.3), and is very close to the temperature with the highest nonthermal velocities 


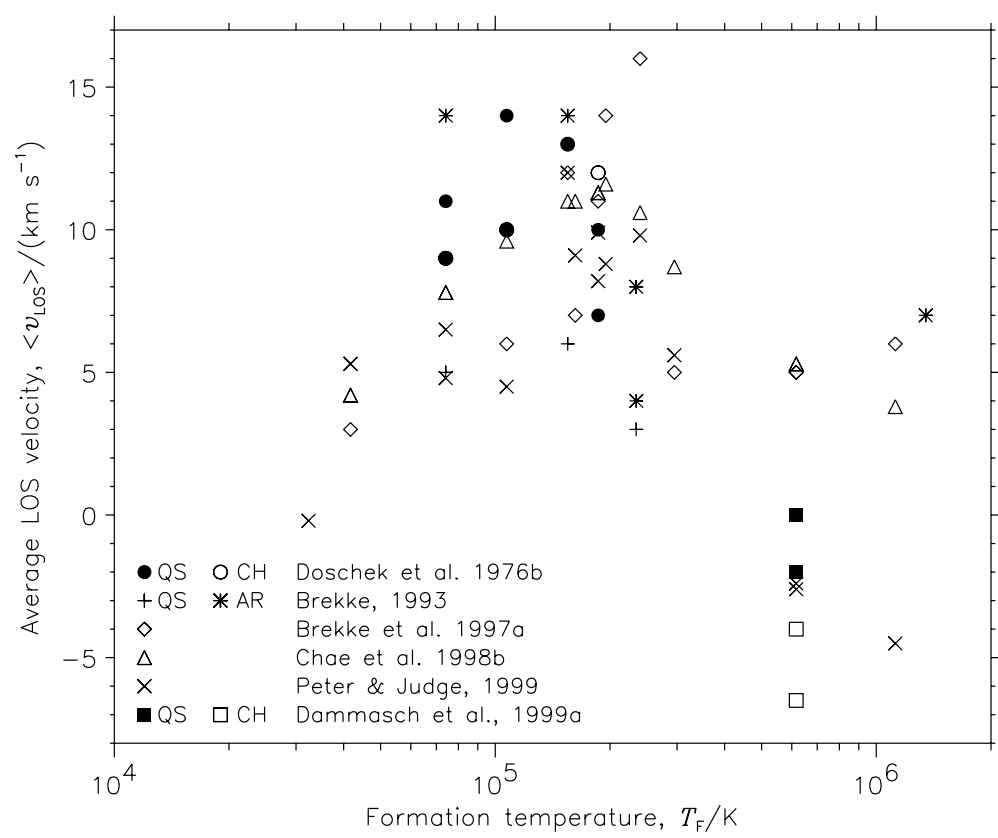

Fig. 9 Average Doppler shifts of solar VUV emission lines. The corresponding LOS velocities (positive: red shift, downwards motion; negative: blue shift, upward motion) are plotted. They were taken from several sources (Doschek et al. 1976b; Brekke 1993; Brekke et al. 1997a; Chae et al. 1998b; Peter and Judge 1999; Dammasch et al. 1999a). The largest shifts occur in transition-region lines at temperatures between $\approx 1 \times 10^{5} \mathrm{~K}$ and $2 \times 10^{5} \mathrm{~K}$. Note the change from a red to a blue shift above $6 \times 10^{5} \mathrm{~K}$ for more recent observations (cf., Sect. 2.1.3.5)

(cf., Sect. 2.1.3.1). In addition to the average red-shift behaviour of transition-region lines, the temporal and spatial variabilities of the plasma flows are of importance. They have been studied by Doyle et al. (2002) with high-cadence observations in C III, O IV, and Ne VIII lines and the result that variations of several kilometres per second can occur on time scales of minutes.

From OSO-8 observations, Lites et al. (1976) also deduced systematic downflows in the chromosphere and transition region from wavelength shifts which, moreover, seem to vary with the line radiance, a result which could be confirmed with SUMER (Judge et al. 1997; Brynildsen et al. 1998; Landi et al. 2000; Dammasch and Wilhelm 2002; Brković et al. 2003) and with CDS (Gallagher et al. 1999). Hansteen et al. (2000) showed that this relationship is restricted to the network lanes and does not apply in inter-network regions.

Differential red shifts of Si IV and C IV emission lines with respect to S I and O I lines have also been observed in International Ultraviolet Explorer (IUE) spectra of late-type stars (Ayres et al. 1983). For instance, an average downflow of $11 \mathrm{~km} \mathrm{~s}^{-1}$ in C IV was measured for Capella. More recently, star observations with the Far Ultraviolet Spectroscopic Explorer (FUSE) confirmed the red shift for other transition region lines (Redfield et al. 2002). Especially the shifts from spectra of $\alpha$ Cen A, which is, as the Sun, also a G2V star, are very similar to the solar values, except for O VI. 


\subsubsection{The Corona}

Above the transition region, the solar corona extends to large distances from the Sun. We review the thermal structure of the corona from the line broadenings/narrowings of heavy ions, and their interpretations in several inhomogeneous structures on the Sun, such as coronal holes, polar coronal plumes, bright points, coronal loops, etc. We also review the status of hydrogen lines as well as waves and oscillations in the corona.

The most puzzling parameter of the corona is its temperature with values of more than 1 MK (Lyot 1937; Grotrian 1939; Edlén 1943) that one would not expect in view of a photospheric temperature of less than $6000 \mathrm{~K}$. The corona eventually expands and transforms into the solar wind (cf., Sect. 3.5). Two types of the solar wind can be identified: a quasistationary fast wind and a transient slow wind (Neugebauer 1991). Ulysses measurements have revealed the typical characteristics of the solar wind at high and low latitudes in interplanetary space (Woch et al. 1997; McComas et al. 1998). Ever since Krieger et al. (1973) discovered that the fast solar wind was emanating from coronal holes, the origin of this outflow from the Sun on open magnetic field lines has been the subject of detailed investigations. The spatial structure of the corona is critically determined by the topology of the magnetic field, and can be described by the open field regions corresponding to dark coronal holes and closed magnetic fields in the form of loops related to relatively bright quiet-Sun areas. Some loops are extending into so-called "helmet streamers".

The structure of the corona is strongly influenced by the solar cycle. During years near the minimum of the sunspot activity, the equatorial latitudes are characterized by closed magnetic regions and the poles are occupied by large coronal holes. During the years near the maximum, the solar atmosphere is much more symmetric and all features are intermingled with many active regions in between (cf., Fig. 12 in Sect. 3.1). Eclipse observations made at various phases of a solar cycle clearly demonstrate these variations (cf., e.g., Sýkora et al. 2002). The responses of the solar wind to the solar activity cycle are reviewed by Marsch (2006b).

The spatial and thermal structures of the solar corona have been studied in great detail with TRACE (cf., Golub et al. 1999). An atlas showing coronal VUV spectra of the quiet Sun, active regions, coronal holes, and flares has been compiled by Curdt et al. (2004) ${ }^{4}$. For a spectral atlas obtained from locations on the disk see Curdt et al. $(2001)^{5}$. All aspects of the physics of the solar corona are treated in a recent book by Aschwanden (2005).

\subsubsection{The Thermal Structure It is often assumed that the electron and the ion tempera-} tures are the same in the transition region, but this assumption clearly breaks down in the low corona, both in streamer regions and in coronal holes (Seely et al. 1997; Tu et al. 1998). A long time ago, theoretical considerations led Lüst et al. (1962) to suggest coronal ion temperatures of $1.6 \mathrm{MK}$ and electron temperatures of $8 \times 10^{5} \mathrm{~K}$.

At greater altitudes, very high (anisotropic) ion temperatures of much more than $10 \mathrm{MK}$ have been detected by coronagraphs on rockets and Spartan as well as by the Ultraviolet Coronagraph Spectrometer (UVCS) on SOHO (Kohl et al. 1980, 1994, 1995a, 1995b, 1996; 1998, 1999; Cranmer et al. 1999a; Esser et al. 1999; Frazin et al. 2003). However, Raouafi and Solanki (2004) demonstrated that the electron density stratification may have a major

\footnotetext{
${ }^{4}$ http://www.mps.mpg.de/homes/curdt/coronal_atlas.pdf.

${ }^{5}$ http://www.mps.mpg.de/homes/curdt/diskatlas_new.pdf.
} 
influence on the observed line width and on the derived anisotropy. The ultraviolet spectroscopy of the extended corona, in particular from UVCS results, has been recently reviewed by Kohl et al. (2006).

All of the radiating ions are, of course, minor constituents of the coronal plasma and do not provide information on the majority population, the protons. Even if $\mathrm{HI}$ lines are observed, it is not trivial to determine the proton parameters. They can only be studied indirectly by observing the hydrogen lines, and by making critical assumptions on the chargeexchange processes. Hydrogen and protons are, however, expected to be closely coupled by charge exchange in coronal holes at heliocentric distances below $2 R_{\odot}$ to $3 R_{\odot}$ (Withbroe et al. 1982). With this assumption and using the higher members of the H I Lyman series, Marsch et al. (1999) found proton temperatures of $2 \times 10^{5} \mathrm{~K}$ at heights of $18 \mathrm{Mm}$ above the limb, and line-width contributions between $20 \mathrm{~km} \mathrm{~s}^{-1}$ and $40 \mathrm{~km} \mathrm{~s}^{-1}$ from turbulence. H I Ly $\alpha$ observations obtained with UVCS indicate line widths of more than $200 \mathrm{~km} \mathrm{~s}^{-1}$ above $2 R_{\odot}$ (reaching $\approx 400 \mathrm{~km} \mathrm{~s}^{-1}$ at $3 R_{\odot}$ ) in polar coronal holes, and somewhat lower values in equatorial coronal holes (Kohl et al. 1997; Miralles et al. 2001).

Section 3 outlines how the interaction between ion-cyclotron waves, generated somewhere in the lower atmosphere, and ions in the coronal-hole plasma is one candidate for the processes heating the corona and accelerating the fast solar wind. In this case, one would expect that the effective temperature of an ion determined from the width of the emitted spectral line is a function of the charge-to-mass ratio, $q / m$, of the ion. Patsourakos et al. (2002) have compiled observations of the effective temperature as a function of $q / \mathrm{m}$ obtained by Hassler et al. (1990), Tu et al. (1998), and Banerjee et al. (2000a). These observations indeed show an increase of the temperature towards lower values of $q / \mathrm{m}$ as expected. SUMER observations in the quiet corona led to similar findings, but above an active region a plot of the thermal velocity versus the charge-to-mass ratio provided a better ordering scheme (Wilhelm et al. 2005a).

In the corona above coronal holes, bi-Gaussian distributions of H I Ly $\alpha, \mathrm{O}$ VI, and Ne VIII profiles with wide wings have been observed by the Spartan Ultraviolet Coronal Spectrometer (UCS), UVCS, and SUMER (Kohl et al. 1996, 1997; Wilhelm, 1998, 1999), which may be related to similar spectra seen in the transition region in funnel and closed-loop structures (Peter 2001) (cf., Sect. 2.1.3.1).

Coronal-hole and equatorial off-limb electron temperature profiles were determined by David et al. (1998). The O VI lines at $17.3 \mathrm{~nm}$ and $103.2 \mathrm{~nm}$ have been observed by CDS and SUMER in the corona above a polar coronal hole. Electron temperatures smaller than $1 \mathrm{MK}$ - decreasing with height—were found with the help of line-ratio studies. They are much less than in the bright equatorial corona of the quiet Sun to be discussed in Sect. 2.1.4.4. Similar observations using the Mg IX line pair at $70.6 \mathrm{~nm}$ and $75.0 \mathrm{~nm}-$ see Keenan et al. (1984) for a theoretical treatment of this ratio-with SUMER resulted in an electron temperature near $8 \times 10^{5} \mathrm{~K}$ in regions between polar plumes and even lower values inside plumes (Wilhelm et al. 1998b). This is in agreement with a Differential Emission Measure (DEM) analysis of CDS polar plume observations that strongly peaked at $7.8 \times 10^{5} \mathrm{~K}$. A plume in an equatorial coronal hole had very similar temperatures (Del Zanna et al. 2003). It was also noted that the window at $19.5 \mathrm{~nm}$ of EIT contains, in addition to Fe XII with a formation temperature of $1.4 \mathrm{MK}$, cooler lines, confirming SUMER observations which do not show any trace of polar plumes in Fe XII (Feldman et al. 2000a). Somewhat higher temperatures of 1.0 MK to $1.1 \mathrm{MK}$ both for plumes and inter-plume regions have been deduced from CDS observations by Young et al. (1999) who saw an increase with height in inter-plume regions and thus confirmed earlier observations by Doschek et al. (1998b). Such an increase was not seen in plumes. Other spectroscopic studies also indicated 
an increase of the electron temperature with height in coronal holes (Doschek et al. 2001) and, specifically, in inter-plume regions (Wilhelm 2006). In Sect. 2.1.3.3, we mentioned the observation by Chiuderi-Drago et al. (1977) that, at low altitudes in coronal holes, the plasma was found not to be homogeneous. Specifically, about $10 \%$ of the gas was hot ( $\approx 2 \mathrm{MK})$ and the rest cooler at $\approx 0.8 \times 10^{5} \mathrm{~K}$.

The low electron temperature (of $\leq 1 \mathrm{MK}$ ) in coronal holes obtained with spectroscopic instruments appears to be in conflict with the so-called "freeze-in" temperatures ${ }^{6}$ determined from charge-state measurements of solar-wind ions. For instance, Ko et al. (1997) obtained a temperature of $\approx 1.5 \mathrm{MK}$ at $1.4 R_{\odot}$. Attempts to reconcile the observations with their interpretations were made by assuming differential flows between ions of the same element (Esser and Edgar 2001), but recent studies conclude that non-Maxwellian electron velocity distributions with an enhanced supra-thermal tail are required in the inner corona to account for the observed charge distributions in the solar wind (Chen et al. 2003). Non-Maxwellian distributions are indeed observed in the solar wind (Marsch 1991), and have long been suggested to cause the observed ionic fractions near the Sun (Owocki and Scudder 1983).

Transient brightenings in quiet-Sun areas have been studied at coronal temperatures by Aletti et al. (2000) using data of the $19.5 \mathrm{~nm}$ channel of EIT. In contrast to the transitionregion lines discussed in Sect. 2.1.3.3, the logarithmic radiance variations of coronal lines are only normally distributed at medium levels and change to a power-law distribution at high radiances. In a study of blinkers and EUV brightenings (cf., Sect. 2.2.1.4), the corona was found to be partially decoupled from the transition region and chromosphere in-quietSun regions (Brković et al. 2001). TRACE $17.1 \mathrm{~nm}$ emission formed at $\approx 1 \mathrm{MK}$ is cospatial with $\mathrm{H} \alpha$ at $\approx 1 \times 10^{4} \mathrm{~K}$, but not with C IV at $1 \times 10^{5} \mathrm{~K}$ (De Pontieu et al. 2003). In a study of quiet-Sun brightenings, Berghmans et al. (1998) compared events observed with EIT in He II $(30.4 \mathrm{~nm} ; 80000 \mathrm{~K})$ and Fe XII $(19.5 \mathrm{~nm}$; $1.4 \mathrm{MK})$. Despite the large difference in the formation temperatures, the relations between the brightening parameters, size, duration, peak radiance, and radiative loss, were rather similar, possibly indicating that the same physical process was responsible for both aspects of the events.

\subsubsection{Coronal Holes, Polar Plumes, and the Fast Solar Wind Coronal holes (Ko-} ronalöcher) in the solar atmosphere have first been identified by Waldmeier $(1951,1957)$ using observations of the green Fe XIV $530.3 \mathrm{~nm}$ line. They are regions of relatively low electron density and, consequently, are characterized by a deficiency in the radiance of coronal emission lines (Munro and Withbroe 1972). In general, there is little change between quiet-Sun and coronal-hole areas in the lines and continua formed below $8 \times 10^{5} \mathrm{~K}$ (cf., Sect. 2.1.3.3), however, coronal holes are visible in the He I and He II lines. The S082A spectroheliograph on Skylab clearly showed a depression of the He II $30.4 \mathrm{~nm}$ network in coronal holes and found very sharp boundaries of coronal holes when seen in this line (Tousey et al. 1973). During the solar activity minimum, large coronal holes are occupying the north and south polar regions of the Sun, as can be seen in Fig. 7.

Bohlin (1977) and Bohlin and Sheeley (1978) reviewed the EUV observations of coronal holes obtained during the Skylab era. They confirmed the anomalous differential rotation of large north-south oriented coronal holes (Timothy et al. 1975) and the open magneticfield topology. The coronal holes were clearly identified with the $\mathrm{M}$ regions introduced by

\footnotetext{
${ }^{6}$ Temperatures at which the relative distributions of the various ionization stages of the solar wind composition are established at a certain height. At greater heights no further changes occur, because of the low collision rate.
} 
Bartels (1940) and Chapman and Bartels (1940) as causes of recurrent geomagnetic storms. The rigid rotation of a coronal hole was also seen by SOHO instruments (Zhao et al. 1999).

Near the limb in coronal holes, an electron density of $\approx 2 \times 10^{8} \mathrm{~cm}^{-3}$ was derived by CDS. It is decreasing to $6 \times 10^{7} \mathrm{~cm}^{-3}$ at a height of $70 \mathrm{Mm}$ (Fludra et al. 1999a). The corresponding temperatures are $7.5 \times 10^{5} \mathrm{~K}$ and $8.5 \times 10^{5} \mathrm{~K}$. The electron density obtained at an altitude of $\approx 7 \mathrm{Mm}$ from SUMER Si VIII line-ratio observations is typically $1 \times 10^{8} \mathrm{~cm}^{-3}$, and it falls off to $\approx 5 \times 10^{6} \mathrm{~cm}^{-3}$ at $200 \mathrm{Mm}$ height (Doschek et al. 1997, 2001; Wilhelm et al. 1998b). Warren and Hassler (1999) used, in addition to Si VIII, several other densitysensitive line ratios and found electron densities in polar coronal holes of $\approx 4 \times 10^{8} \mathrm{~cm}^{-3}$ between $7 \mathrm{Mm}$ and $14 \mathrm{Mm}$ above the limb. This high density is probably due to a substantial contribution of plume material to the LOS measurements.

Polar plumes can be seen as prominent features of polar coronal holes in the visible and at VUV wavelengths in spectral lines with formation temperatures $\leq 1 \mathrm{MK}$ (van de Hulst 1950a, 1950b; Bohlin et al. 1974; Fisher and Guhathakurta 1995; DeForest et al. 1997; Wilhelm et al. 1998b). Height profiles of the electron density in plumes had been obtained before from MSSTA observations, yielding values which were about a factor of two larger than the more recent ones (Walker et al. 1988). Using CDS (GIS) observations of plasma structures on the disk, Del Zanna et al. (2003) obtained a density of $n_{\mathrm{e}} \simeq 1.2 \times 10^{9} \mathrm{~cm}^{-3}$ for a plume, about twice the value of the surrounding coronal hole plasma. Wilhelm (2006) studied a south-polar coronal hole in May 2005 and found a density ratio of $\approx 5$ between plumes and inter-plume regions, with plume material occupying less than $8 \%$ of the intervening space along the LOS.

With Spartan 201-1 very broad H I Ly $\alpha$ profiles were observed in the corona above polar coronal holes, and indications were found that a polar plume was cooler than its environment (Kohl et al. 1995a). UVCS and SUMER observations demonstrated that the widths of VUV emission lines are narrower within plumes than outside (Antonucci et al. 1997; Corti et al. 1997; Hassler et al. 1997; Banerjee et al. 2000a). MSSTA observations indicated that most plumes have a unipolar magnetic structure with electron densities of $1 \times 10^{8} \mathrm{~cm}^{-3}$ at $1.05 R_{\odot}$ and $2 \times 10^{7} \mathrm{~cm}^{-3}$ at $1.5 R_{\odot}$. No conclusive answer on flows in plumes could be obtained by Allen et al. (1997).

In images of EIT and the Large Angle Spectroscopic Coronagraph (LASCO; Brueckner et al. 1995) individual plume structures could be identified between $1.1 R_{\odot}$ and $3 R_{\odot}$. They showed super-radial expansion and were episodic in nature, lasting $\approx 24 \mathrm{~h}$, but recurring at the same location with respect to the Sun for longer periods (DeForest et al. 2001).

A study of the relationship of magnetic fields as seen in magnetograms from KPNSO and polar plumes in Fe IX at $17.1 \mathrm{~nm}$ observed by EIT revealed that polar plumes occur on field lines of the dominant-polarity in contact with the minority polarity in polar coronal holes during the solar activity minimum (Wang et al. 1997). Near the bases of the plumes, a pattern of bright network structures in He II $30.4 \mathrm{~nm}$ is found. The darkest parts of polar coronal holes coincide with areas where there is little magnetic flux of either polarity or only the dominant one. The conclusion reached from these observations is that most of the open flux does not carry plume material and, therefore, plumes are not the main source of the fast solar wind. An increase in the magnetic reconnection rate will lead to an increase in energy deposition, which cannot be radiated away in the low-density coronal hole, and will thus create a plume with a typical evaporation time scale of $\approx 6 \mathrm{~h}$. At the end of the reconnection process, the plume will decay on a cooling time scale of $\approx 4 \mathrm{~h}$ (Y.-M. Wang 1998).

Radiance increases can occur and propagate outwards along plumes (with speeds of about $100 \mathrm{~km} \mathrm{~s}^{-1}$ ) and recur quasi-periodically on time scales of $\approx 10 \mathrm{~min}$ (DeForest and Gurman 1998). These variations have been simulated as being trapped slow magnetosonic waves by 
Ofman et al. (1999). Waves in plumes with periods of $10 \mathrm{~min}$ to $25 \mathrm{~min}$ were also found by Banerjee et al. (2000b), and compressional waves with even longer periods in inter-plume regions (Banerjee et al. 2001b).

High spectral resolution was required to measure the Doppler shifts of emission lines in the accelerating solar wind near the source regions. First indications of outflow with speeds a little above $10 \mathrm{~km} \mathrm{~s}^{-1}$ were found for $\mathrm{Mg}^{8+}, \mathrm{Mg}^{9+}$, and $\mathrm{Si}^{10+}$ ions by Cushman and Rense (1976) in a coronal hole or, more likely, in the neighbourhood of a coronal hole (Cushman and Rense 1977). Mariska (1978) predicted an outflow speed of $15 \mathrm{~km} \mathrm{~s}^{-1}$ at $1.08 R_{\odot}$ on the basis of the observed EUV-emission gradients in a coronal hole and a comparison with solar wind parameters at the Earth. A comparison of emission-measure curves deduced from coronal-hole with quiet-Sun spectra taken with the HCO spectroheliometer indicated outflow at a speed of $\approx 2 \mathrm{~km} \mathrm{~s}^{-1}$ in coronal holes (Raymond and Doyle 1981). Rottman et al. (1982) observed maximal blue shifts in coronal holes relative to quiet-Sun regions for the $\mathrm{OV}$ and $\mathrm{Mg}$ X $62.5 \mathrm{~nm}$ lines. These shifts are consistent with outflow speeds in coronal holes of $7 \mathrm{~km} \mathrm{~s}^{-1}$ and $12 \mathrm{~km} \mathrm{~s}^{-1}$, respectively, if the remainder of the solar disk is at rest. Warren et al. (1997b), using SUMER observations, also found a blue shift of about $15 \mathrm{~km} \mathrm{~s}^{-1}$ in coronal holes relative to quiet-Sun regions. Concentrated outflow of $\mathrm{Ne}^{7+}$ ions could be identified along coronal-hole network lanes and, in particular, near their intersections (Hassler et al. 1999). However, Aiouaz et al. (2005a) located the maximum outflow near the network boundaries ${ }^{7}$, and Popescu et al. (2004) identified outflow regions there in the relatively cool O III $70.3 \mathrm{~nm}$ line. Above the chromospheric network, the outflow appears to be strongest in the dark regions of the coronal holes and not in the bright polar plumes (Wilhelm et al. 1998b, 2000; Giordano et al. 2000; Patsourakos and Vial 2000). Wiegelmann et al. (2005) and Tu et al. (2005a, 2005b) identified the locales of strong blue shifts as the footpoint regions of funnels in coronal holes. An observation of a small plume in an equatorial coronal hole has been reported by Del Zanna and Bromage (1999). As for polar plumes, a reduced temperature was found in this plume with respect to its environment.

An interesting technique for measuring flow speeds in the extended corona is called Doppler dimming (Withbroe et al. 1982; Kohl and Withbroe 1982). It was first suggested by Hyder and Lites (1970) for studying the motion of prominences, and depends on the variation of the resonantly scattered radiation as a function of the flow speed of the plasma relative to the illuminating radiation source. Results obtained by UVCS showed that the acceleration of the fast solar wind occurred close to the Sun, and that speeds of $400 \mathrm{~km} \mathrm{~s}^{-1}$ were reached within $2 R_{\odot}$ (Cranmer et al. 1999b). Patsourakos and Vial (2000) demonstrated that speeds of about $70 \mathrm{~km} \mathrm{~s}^{-1}$ can be found in the dark regions of polar coronal holes at altitudes of $35 \mathrm{Mm}$. Antonucci et al. (2000) examined UVCS observations obtained in a polar coronal hole on 21 May 1996, during the SOHO Joint Observing Programme (JOP) 2. They concluded, from a Doppler-dimming analysis of the H I Ly $\alpha$ and O VI $103.2 \mathrm{~nm}$ and $103.7 \mathrm{~nm}$ lines, that the outflow speeds of hydrogen and oxygen ions were consistent with the proton speed as derived from flux-conservation arguments, if a cool and tenuous coronal plasma was assumed for the inter-plume regions, in which the outflow was thought to occur. Teriaca et al. (2003) used SUMER and UVCS observations of the same lines up to heliocentric distances of $2 R_{\odot}$. They also identified the inter-plume regions as the sources of the fast solar wind, whereas the polar plumes appeared to be rather static. The outflow speed in inter-plume regions increased to about $150 \mathrm{~km} \mathrm{~s}^{-1}$ between the limb and $2 R_{\odot}$. In contrast to these and many other results, Gabriel et al. (2003) concluded on the basis of CDS and

\footnotetext{
${ }^{7}$ An erratum (Aiouaz et al. 2007) did not affect this conclusion.
} 
SUMER measurements that the outflow speed in polar plumes was significantly higher than in inter-plume regions; for instance, $\approx 80 \mathrm{~km} \mathrm{~s}^{-1}$ in a plume and about half this speed in an inter-plume region, both at $1.25 R_{\odot}$. Considering the surface distribution of plumes and inter-plume areas, the authors further deduced that each region contributed about one half to the fast wind. With UVCS observations, Gabriel et al. (2005) found that the outflow speed in plumes was higher than in inter-plume regions below $1.6 R_{\odot}$ and fell below inter-plume values at greater heights. The conflicting results of these studies are even more surprising in view of the fact that all authors used the same technique.

A specific aspect of the Doppler-dimming method is the pumping of the O VI $103.7 \mathrm{~nm}$ line by the nearby C II $103.7 \mathrm{~nm}$ line (Noci et al. 1987). Note that not only the $103.700 \mathrm{~nm}$ line can pump the O VI line, but also the C II $103.634 \mathrm{~nm}$ line will become of importance at higher speeds (Warren et al. 1998; Li et al. 1998).

The magnetic field undoubtedly plays a critical rôle for all plasma processes in the corona. However, there are no reliable measurements available for the coronal magnetic field. Linear polarization measurements of the O VI line at $103.2 \mathrm{~nm}$ by SUMER presented by Raouafi et al. (2002) are thus of interest, as they provide constraints on the magnetic field and solar-wind flow in polar coronal holes.

Woo et al. (2004) argued that the separation of the corona into open regions producing the fast solar wind streams and closed regions has to be replaced by a concept that allows the corona to open up outside coronal holes as well. The conditions for magnetic confinement and release of the coronal plasma would then determine whether fast or slow wind will be generated.

With increasing solar activity, the polar coronal holes disintegrate and spread over the whole Sun, giving rise to so-called equatorial coronal holes, which in some cases are still connected to the polar coronal holes. These holes rotate nearly rigidly with the Sun and not differentially as the photosphere (Timothy et al. 1975). An increased number of bidirectional jets occurred at the boundaries of the October 1996 equatorial coronal hole. This observation supports the idea that magnetic reconnection is instrumental in achieving the required reconfiguration of the coronal magnetic field (Madjarska et al. 2004).

Most of the coronal-hole outflow studies have been performed in polar coronal holes, which are well defined during the minima of the solar magnetic activity. Of the few investigations related to equatorial coronal holes we discuss here some of the results obtained with UVCS, LASCO, SUMER, and CDS. Very similar characteristics of the H I Ly $\alpha$ and O VI $103.2 \mathrm{~nm}$ emission lines in polar and equatorial coronal holes were reported by Dobrzycka et al. (1999). Both types are expanding superradially, but there appears to be a lower $\mathrm{O}^{5+}$ outflow speed in equatorial coronal holes. The temperature of an equatorial coronal hole observed in October 1996 with CDS and the Nancasy Radioheliograph was found to be comparable to those in polar holes (Chiuderi-Drago et al. 1999). Empirical models for polar and equatorial coronal holes derived from $\mathrm{SOHO}$ and ground-based data confirmed that the outflow speed of $\mathrm{O}^{5+}$ ions is much lower in equatorial coronal holes. Since in situ observations of the solar wind showed comparable asymptotic speeds, the acceleration seems to occur at greater altitudes in equatorial than in polar coronal holes (Miralles et al. 2001, 2004).

SUMER raster scans in 1999 obtained in various transition-region lines and in Ne VIII, which has a formation temperature of $6.3 \times 10^{5} \mathrm{~K}$, have been compared with magnetograms from the MDI and from KPNSO. The plasma in structures above concentrations of unipolar magnetic fields seems to be flowing out, and to have usually broader line widths, i.e., higher effective ion temperatures (Xia et al. 2003; Xia et al. 2004). There is no significant difference between equatorial and polar coronal holes. In agreement with these findings, McIntosh et al. (2006) saw the highest Doppler velocities in regions with unbalanced magnetic polarities. 
2.1.4.3 Bright Points The smallest bipolar magnetic features in the corona are called bright points, which are especially prominent in coronal holes, but are also seen in the quiet Sun (Tousey et al. 1973). They have been studied in detail by Golub et al. $(1974,1977)$ and Golub (1980), who found that bright points had a mean lifetime of $8 \mathrm{~h}$ in X rays and were associated with emerging magnetic flux. The spacing between the magnetic poles increased with time. The lifetime of bright points in the EUV is on average $20 \mathrm{~h}$ (Zhang et al. 2001a). Changes in the fine structure of bright points were observed by Sheeley and Golub (1979). Individual miniature loops of $\approx 2.5 \mathrm{Mm}$ in diameter and $12 \mathrm{Mm}$ in length had lifetimes of $\approx 6$ min. Radiance variations at such a time scale were also observed by Habbal and Withbroe (1981) in substructures of bright points.

With the sounding rocket payload Normal Incidence X-ray Telescope (NIXT), Golub et al. (1990) observed, with very high spatial resolution, a few bright points at $6.35 \mathrm{~nm}$ in the lines of $\mathrm{Mg} \mathrm{X}$ and Fe XVI and found bipolar magnetic structures. Comparison of NIXT observations with those of the Soft X-ray Telescope (SXT) on Yohkoh indicated that these compact loop structures had a low filling factor (Di Matteo et al. 1999). MSSTA observations in Fe XII also resolved bright points as tiny loops (Kankelborg et al. 1996). In a review of bright point observations, Habbal (1992) emphasized their arcade structure of loops with different temperatures and emission variations on the time scale of minutes.

Using HRTS observations, Webb et al. (1993) and Moses et al. (1994) also established an association between bright points and magnetic dipoles, but found converging features of preexisting flux to be cancelling rather than flux emerging in a bright point. The footpoints appeared bright in H I Ly $\alpha$. The observed Doppler shifts in C IV indicated velocities of $\approx 40 \mathrm{~km} \mathrm{~s}^{-1}$, but bright points do not correspond directly to explosive events (cf., Sect. 2.2.1.3). EIT, MDI, CDS, and SUMER observations analysed by Madjarska et al. (2003) essentially confirmed these results, in particular, it was shown that the observed bright point was related to the cancellation of magnetic flux. The bright point became visible when the separation of the magnetic poles was $7 \mathrm{Mm}$ and lasted until one of the polarities had disappeared. The bright point was characterized by many short-lived $(\approx 10 \mathrm{~min})$ radiance increases and by corresponding Doppler shifts towards longer wavelengths (i.e., down flows). Vilhu et al. (2002) also observed strong microflaring of a bright point in emission lines with formation temperatures between $2 \times 10^{4} \mathrm{~K}$ and $5 \times 10^{5} \mathrm{~K}$. Electron densities in bright points have been determined by Ugarte-Urra et al. (2005) using line-ratio data from CDS. They found values between $1 \times 10^{9} \mathrm{~cm}^{-3}$ and $1 \times 10^{10} \mathrm{~cm}^{-3}$ at formation temperatures of 1.3 MK to 2 MK. Very recently, Brosius et al. (2007) found Doppler velocities above a bright point of $\pm 15 \mathrm{~km} \mathrm{~s}^{-1}$ (He II) and $\pm 35 \mathrm{~km} \mathrm{~s}^{-1}$ (Fe XVI).

\subsubsection{The Equatorial Corona, Coronal Loops, and the Slow Solar Wind Flows of cool} plasma in coronal loops were first observed by Foukal (1978) from condensations near their apices, and were thought to indicate forced convection. MSSTA observations in 1991 showed that loops with constant cross-sections are much more typical than loops with wider cross-sections at the top of the loop. O'Neal et al. (1993) reported these findings in an abstract and O'Neal (1995) in his thesis. Yohkoh observations near the top of coronal loops, where the temperatures are highest, gave values of $\approx 4$ MK (cf., Kano and Tsuneta 1996). With UVCS observations, a difference in the H I Ly $\alpha$ and O VI streamer morphology was discovered, in the sense that the Ly $\alpha$ image depicted a rather simple geometry, whereas in O VI several substructures were seen (Noci et al. 1997).

Raymond et al. (1997), as well as Feldman et al. (1999a), found an isothermal electron temperature of $1.3 \mathrm{MK}$ in the equatorial streamer plasma between $1.05 R_{\odot}$ and $1.5 R_{\odot}$, where such a temperature was confirmed with UVCS observations by Parenti et al. (2000). 
But closer to the limb an increase of more than $1 \times 10^{5} \mathrm{~K}$ starting near $1.1 \mathrm{MK}$ was deduced from CDS line-ratio measurements. The electron density decreased from $\approx 2 \times 10^{8} \mathrm{~cm}^{-3}$ to $1.6 \times 10^{7} \mathrm{~cm}^{-3}$ over this distance range. A similar temperature of $T_{\mathrm{e}}=1.44 \mathrm{MK}$ in a quiet coronal streamer between $1.05 R_{\odot}$ and $1.35 R_{\odot}$ was deduced from an emission-measure analysis of silicon lines (Warren and Warshall 2002). This temperature of the coronal plasma and the resulting emission lines were used by Landi et al. (2002) for an assessment of the CHIANTI database. David et al. (1998) deduced somewhat higher temperatures from CDS and SUMER O VI observations. The iron lines Fe IX to Fe XV observed by CDS within the cores of coronal streamers yielded higher temperatures during the solar maximum than minimum conditions (Foley et al. 2002). Using the radiance ratios of the iron lines, they found a temperature increase from $\approx 1.2 \mathrm{MK}$ at $1.05 R_{\odot}$ to $\approx 1.4 \mathrm{MK}$ at $1.3 R_{\odot}$ during the minimum in 1996, and values about 0.8 MK higher during the solar maximum in 1999.

Quiet-Sun coronal loops, which may exceed heights of $700 \mathrm{Mm}$, can survive for days. Since the electron density greatly decreases between the base and the top of such a loop, differential settling of the elements will occur, as the plasma turbulence is fairly small. In comparison with elements having atomic mass numbers from 20 to 28 (neon to silicon), the iron ions are by a factor of two to three less abundant at $1.5 R_{\odot}$ than near the photosphere (Feldman et al. 1999a). Similar factors are reported for larger heights from UVCS observations (Raymond et al. 1997). For a more general discussion of coronal elemental abundances see Sect. 2.3.2.

Feldman et al. (1999b) combined observations of the spectrograph S082A with TRACE and SUMER measurements of VUV lines with formation temperatures between $4 \times 10^{4} \mathrm{~K}$ and 1.4 MK. The morphology of the solar atmosphere was described by them as a hierarchy of isothermal loop structures. The cooler loops are concentrated along chromospheric network lanes, whereas the high-temperature loops seem to form a canopy over the cold structures.

Frazin et al. (2003) used SOHO observations from UVCS and LASCO to study the $\mathrm{O}^{5+}$ velocity distributions and outflow speeds in an equatorial streamer during the sunspot minimum in 1996. They found anisotropic distributions in the streamer legs and stalks, with outflow speeds between $40 \mathrm{~km} \mathrm{~s}^{-1}$ and $\approx 100 \mathrm{~km} \mathrm{~s}^{-1}$ in the range from $4.6 R_{\odot}$ to $5.1 R_{\odot}$.

Coronal loops are basically defining the magnetically closed corona. Using a $17.1 \mathrm{~nm} /$ $19.5 \mathrm{~nm}$ filter ratio technique to examine TRACE data obtained near the solar limb, Lenz et al. (1999) found that there was very little temperature variation along the loops. In fact, they appeared to be isothermal at $1.3 \mathrm{MK}$. The rôle of filamentation in determining the temperature was studied by Reale and Peres (2000). Further investigations with the help of the $17.1 \mathrm{~nm}$ and $19.5 \mathrm{~nm}$ passbands of TRACE and model calculations yielded consistent density and temperature values in coronal loops. Evidence was provided that hot $(\approx 5 \mathrm{MK})$ and cold $\left(\approx 2 \times 10^{5} \mathrm{~K}\right)$ fibrils together form a loop (Testa et al. 2002). A disturbance observed by TRACE at one footpoint of a cold loop led to a strong red-shifted emission of $\mathrm{N} \mathrm{V}$ at $123.8 \mathrm{~nm}$ emission at the other footpoint (Doyle et al. 2006a). CDS observations followed by a DEM analysis yielded a broad temperature distribution for a coronal loop-not consistent with an isothermal plasma - whereas a narrow DEM curve was found in an adjacent loop (Schmelz et al. 2007a). The authors concluded that CDS measurements can constrain coronal-loop temperatures and that filter ratios of the $17.1 \mathrm{~nm}$ and $19.5 \mathrm{~nm}$ windows may not be sufficient for this purpose. Schmelz et al. (2007b) furthermore showed that even with the help of the $28.4 \mathrm{~nm}$ filter the coronal plasma temperatures cannot adequately be determined.

The quiet equatorial corona at low altitudes is cooler $(\approx 1 \mathrm{MK})$ than the corona at midlatitudes $(\approx 1.5 \mathrm{MK})$ according to SUMER, EIT, and LASCO/C1 observations (Wilhelm et 
al. 2002a). Near the equator high-density $\left(n_{\mathrm{e}} \approx 5 \times 10^{9} \mathrm{~cm}^{-3}\right)$ transition-region plasma and low-density $\left(n_{\mathrm{e}} \approx 2 \times 10^{8} \mathrm{~cm}^{-3}\right)$ coronal plasmas are present at the same height of $5 \mathrm{Mm}$.

Electron density diagnostics using line ratios of various iso-electronic sequences were performed by Laming et al. (1997). In the quiet low-altitude corona, as observed in 1996 above the west limb $(\approx 20 \mathrm{Mm})$, most of the measurements gave consistent results of several $10^{8} \mathrm{~cm}^{-3}$.

In comparison to the generation of the fast solar wind from coronal holes, relatively little is known about the slow solar wind originating from closed magnetic regions in the equatorial streamer belt, which is well developed during sunspot minimum periods. Using LASCO white-light images, Sheeley et al. (1997) tracked small coronal features in the slow solar wind and found their source near $3 R_{\odot}$ and an acceleration of $\approx 4 \mathrm{~m} \mathrm{~s}^{-2}$ to a speed of $300 \mathrm{~km} \mathrm{~s}^{-1}$ near $25 R_{\odot}$. At low heights, there is no significant outflow in the core of coronal streamers, as UVCS and LASCO observations indicate. The outflow providing the mass flux for the slow solar wind starts near the streamer legs at the boundaries of closed and open magnetic field regions (Habbal et al. 1997; Woo and Martin 1997; Strachan et al. 2002; Antonucci et al. 2005).

\subsubsection{Coronal Oscillations and Waves Broadening of emission lines can provide, albeit} in an indirect way, information on unresolved wave activity in the corona. Hassler et al. (1990) observed excessive line broadening of the $\mathrm{Mg} \mathrm{X}$ lines at $60.9 \mathrm{~nm}$ and $62.5 \mathrm{~nm}$ above the limb, indicating high effective ion temperatures, probably caused by unresolved wave activity. Harrison et al. (2002) deduced from CDS observations of the near-equatorial quiet corona a narrowing of the $\mathrm{Mg}$ X $62.5 \mathrm{~nm}$ line with height above the limb, which could not be confirmed by SUMER observations (Doschek and Feldman 2000; Wilhelm et al. 2004b). Joint observations by CDS and SUMER in 2003 showed a relatively constant line width with height (Wilhelm et al. 2005a).

Roberts et al. (1984) showed that magneto-acoustic oscillations are expected in coronal inhomogeneities - for instance, in coronal loops-and suggested that the physical conditions of the corona can be studied by investigating these oscillations. Thus, the idea of coronal seismology was born. Transverse coronal-loop oscillations have been detected by TRACE (Aschwanden et al. 1999a, 2002, 2003a) and interpreted as global kink-mode oscillations with periods of $\approx 280 \mathrm{~s}$. These oscillations can be excited by strong flares, but occasionally also by filament eruptions (Schrijver et al. 2002). Nakariakov and Ofman (2001) estimated the magnetic field strength in an oscillating loop to be $\approx 1.3 \mathrm{mT}$. Slow magnetoacoustic disturbances propagating with speeds of roughly $100 \mathrm{~km} \mathrm{~s}^{-1}$ have been observed by the EIT and TRACE instruments in active-region loops (Robbrecht et al. 2001).

Active-region loop oscillation studies, made by O'Shea et al. (2001) in transition-region and coronal emission lines seen with CDS, indicated that certain periods are favoured for each line. However, the 5 min oscillations of the photosphere are in general present in all lines. In coronal lines, the velocity oscillations are more prominent than the radiance oscillations. This is not the case in transition-region lines, and thus it appears as if non-compressive modes, which do not affect the radiance, are generated in the corona and not in the transition region.

Using SUMER, EIT, and SXT observations, Kliem et al. (2002) found LOS velocities of $\pm 150 \mathrm{~km} \mathrm{~s}^{-1}$ in C II and Fe XXI lines during an M3 flare with velocity oscillations with periods from $4 \mathrm{~min}$ to $8 \mathrm{~min}$. These damped oscillations were further studied by Wang et al. $(2002,2003)$ and Ofman and Wang (2002), who found phase shifts of $90^{\circ}$ between radiance and velocity oscillations, consistent with the signature of a standing slow-mode wave in a hot loop. 

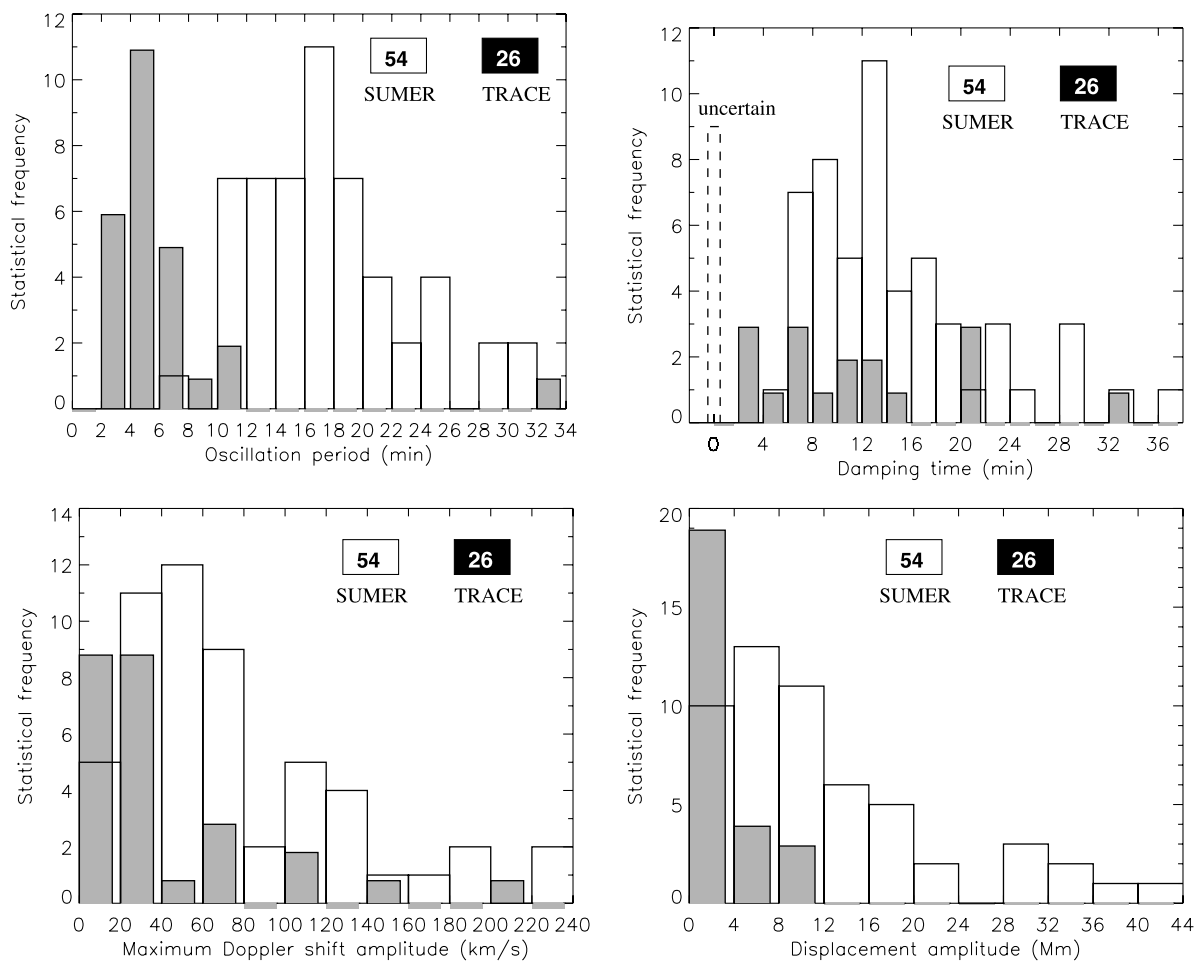

Fig. 10 Comparison of SUMER and TRACE loop-oscillation parameters. The TRACE oscillations (26 cases) have much shorter periods and smaller displacement amplitudes than the SUMER oscillations (54 cases) (from Wang 2004)

Observations and interpretations of coronal-loop oscillations have recently been reviewed by Wang (2004). Figure 10 shows his comparison of the physical parameters of SUMER and TRACE oscillations. There are significant differences in the periods and displacement amplitudes for oscillations that are either observed with TRACE at temperatures of $\approx 1 \mathrm{MK}$ or with SUMER in hot iron lines with formation temperatures of more than $6 \mathrm{MK}$. Vertical oscillations of coronal loops could also be observed by TRACE in its $19.5 \mathrm{~nm}$ channel (Wang and Solanki 2004).

Evidence for downward-travelling compressive waves from the corona through the transition region was presented by Judge et al. (1998), who analysed the density-sensitive line pair of O IV at $139.9 \mathrm{~nm}$ and $140.1 \mathrm{~nm}$.

A very different phenomenon recorded by EIT was presented by Thompson et al. (1999). Initiated by active-region transients, large-scale coronal waves were propagating as EUV emission fronts at a speed of $\approx 250 \mathrm{~km} \mathrm{~s}^{-1}$ across the solar disk. Whether or not these "EIT waves" can be identified with Moreton waves (Moreton 1961) could not be decided with certainty in early studies. Later studies analyzing many of these events (cf., Biesecker et al. 2002; Chen et al. 2005) indicated that nearly all the waves were associated with Coronal Mass Ejections (CME), but that the correlation with flares (cf., Sect. 2.2.2.6) is much weaker. Since Moreton waves are associated with flares (Uchida 1968), EIT waves appear to be a different type of phenomenon, although both waves can occur together in some cases. Active regions or coronal holes seem to inhibit somewhat the wave propagation. 


\subsection{Solar Atmospheric Structures and Events}

The solar atmosphere is very inhomogeneous and dynamic, and can only approximately be treated as a horizontally stratified medium. Section 2.1 has, therefore, to be supplemented by a description in which the features and events discussed in the previous sections are considered as disturbances of the layered but otherwise uniform atmosphere.

\subsubsection{Small-Scale Transients}

Presenting the chromospheric network as a site for the dynamical processes, and which is eventually responsible for the coronal heating and solar wind acceleration, we review spicules and macrospicules, jets and tornadoes, explosive events, blinkers, microflares and nano-flares from the VUV data. Not only does the chromospheric network have a complicated magnetic structure, it is also the site of all these small-scale dynamical processes. Thus, any description of the solar atmosphere in terms of average properties is incomplete without consideration of the fine-scale structure and its dynamics.

Rapid variations of the radiances of VUV lines were first observed on Skylab (Vernazza et al. 1975). Shine et al. (1976), analyzing OSO-8 data, found wider line profiles (by a factor of 1.08) of C IV $154.8 \mathrm{~nm}$ in network lanes than in cells. Athay and White (1980) described OSO-8 observations in Si II and C IV, which had temporal and spatial fluctuations on all time scales. The activity in the network reveals itself most clearly in explosive events, jets and blinkers that can best be seen in transition-region lines.

It is impossible to cover all aspects of this topic here, and thus only some examples will be selected. A short review on transition-region dynamics was given by Innes (2004). The episodic energy releases leading to explosive events and other small-scale transients have often been related to the coronal heating problem, but without any success if only the particle energy was taken into account. It appears necessary to consider, in addition, the magnetic energy associated with reconnection and the wave energy generated during such events (Axford and McKenzie 1992; Tu et al. 1998; Aschwanden et al. 2000a; Wilhelm et al. 2002b), but a quantitative assessment is not yet available. The waves are strong candidates for accelerating coronal ions preferentially perpendicular to the magnetic field via ion-cyclotron resonance interaction (Marsch and Tu 1997a, 1997b; Cranmer et al. 1999b). If this process could be confirmed, it would help in resolving the long-standing coronal heating problem (cf., Sect. 3.4.2).

2.2.1.1 Spicules and Macrospicules Spicule observations and theoretical concepts of the spicule formation were reviewed by Sterling (2000). Their small sizes and short lifetimes place severe constraints on the detailed observations required to conceive a satisfactory spicule model. As mentioned in Sect. 2.1.3.5, Pneuman and Kopp (1978) related the observed average downflow in the transition region to the return flow of spicular material. Dere et al. (1987) concluded that, in all likelihood, the transition-region spicules are outward extensions of chromospheric spicules.

Here we consider mainly VUV observations of spicules, but leave open their relation to events seen in the visible. Xia et al. (2005) studied time series of spicules and macrospicules with SUMER. They identified a "falling after rising" process in some of the spicules. It must be remembered, however, that the LOS geometry near the limb is not very advantageous for the observation of vertical motions. Spicules observed in various VUV emission lines, which are formed at temperatures between $3 \times 10^{4} \mathrm{~K}$ and $6 \times 10^{5} \mathrm{~K}$, exhibited an increase in their diameters with growing temperature. Above $6 \times 10^{5} \mathrm{~K}$ the spicule signature is no longer 
discernible (Budnik et al. 1998). Guided by VUV observations of spicules with SUMER, which showed significant red and blue Doppler shifts as characteristic features, Wilhelm (2000b) questioned spicule models that are based on field-aligned material propagation and favoured reconnection followed by a sling-shot effect.

Macrospicules were discovered by Bohlin et al. (1975) in He II $30.4 \mathrm{~nm}$ spectroheliograms of the slitless spectrograph S082A. They had lengths of up to 50" and lifetimes between $5 \mathrm{~min}$ and $40 \mathrm{~min}$. Their occurrence is restricted to polar coronal holes, and their inclination decreases away from the poles in close resemblance to the orientation of polar plumes. This is seen as evidence that both features are controlled by the diverging magnetic field of the polar caps. A comparison of macrospicules observed in $\mathrm{H} \alpha$ by the Big Bear Solar Observatory and in He II $30.4 \mathrm{~nm}$ by EIT by Wang (1998) showed that all identified He II events had counterparts in $\mathrm{H} \alpha$, although their morphologies are different. In polar coronal holes many $\mathrm{H} \alpha$ macrospicules do not have a corresponding He II $30.4 \mathrm{~nm}$ event.

A macrospicule observation with CDS showed high LOS velocities reaching a plateau of $200 \mathrm{~km} \mathrm{~s}^{-1}$ at an altitude of about $18 \mathrm{Mm}$ (Pike and Harrison 1997). Another CDS observation of a macrospicule in a south-polar coronal hole in March 1998 indicated electron densities of the order of $10^{10} \mathrm{~cm}^{-3}$ and a temperature of $\leq 3 \times 10^{5} \mathrm{~K}$, with parameters in the background plasma of $1 \times 10^{8} \mathrm{~cm}^{-3}$ and $\approx 1 \mathrm{MK}$. The outflow speed near the limb was $\geq 80 \mathrm{~km} \mathrm{~s}^{-1}$ (Parenti et al. 2002).

2.2.1.2 Jets and Tornadoes Jets in the corona have been discovered in high-resolution observations with a tandem Wadsworth spectrograph (Brueckner and Bartoe 1983). Shibata et al. (1996) discovered coronal X-ray jets in association with bright-point activity, and interpreted this phenomenon as reconnection between newly emerging magnetic flux and preexisting coronal field.

Associated with a flare and a CME, CDS spectroscopic measurements of the O $\mathrm{v}$ emission line showed a plasma ejection, with an outward flow at a speed of $\approx 150 \mathrm{~km} \mathrm{~s}^{-1}$ and superimposed a rotational motion at a speed of $\pm 350 \mathrm{~km} \mathrm{~s}^{-1}$ (Pike and Mason 2002). On a smaller scale, a rotational event was observed by Wilhelm et al. (2002b). The spectroscopic signatures of rotating structures were thoroughly studied by Rompolt (1975). Harrison et al. (2001) observed with CDS at the limb a fan-like jet in $\mathrm{Mg}$ X followed by a rotating jet seen in O V. Speeds of $\approx 380 \mathrm{~km} \mathrm{~s}^{-1}$ (red) and $\approx 190 \mathrm{~km} \mathrm{~s}^{-1}$ (blue) were measured with indications of an acceleration. Magnetic reconnection of low-lying loops with open field lines were proposed as explanation.

Chromospheric jets seen by HRTS in the C I lines near $156 \mathrm{~nm}$ and $166 \mathrm{~nm}$ have extremely fine structures and show strong red and blue Doppler shifts (Dere et al. 1983; Brueckner and Bartoe 1983). Inclined streaks in spectra of the CIV $154.8 \mathrm{~nm}$ emission line indicate local acceleration of plasma along its propagation path in the transition region. Typical accelerations are between $10 \mathrm{~km} \mathrm{~s}^{-2}$ and $20 \mathrm{~km} \mathrm{~s}^{-2}$ (Wilhelm et al. 2002b). In close proximity to this activity, rotating events and coronal jets with LOS velocities of more than $200 \mathrm{~km} \mathrm{~s}^{-1}$ have been observed.

\subsubsection{Explosive Events Explosive events have been observed with high spectral and spa-} tial resolutions by HRTS and SUMER (cf., Brueckner and Bartoe 1983; Dere et al. 1989b; Kjeldseth-Moe and Cheng 1994; Moses et al. 1994; Innes et al. 1997a, 1997b; Innes 2001). Observations of the line profile of CIV at $154.8 \mathrm{~nm}$ with HRTS revealed that, in general, the shapes of the line during the events can be described by three Gaussian profiles with one component at rest and the others shifted by $\approx 10 \mathrm{pm}$ to either red or blue (Dere et al. 1981). Based on these observations, it was first suggested that the events are produced 
by radiative cooling. However, most of the later investigations indicated that this activity, on spatial scales of a few thousand kilometres and temporal scales of a few minutes, is a consequence of low-altitude magnetic field-line reconnection. Explosive events are concentrated at the edges of strong network fields in quiet-Sun regions (including coronal holes), or within weaker field regions in the network. They are not associated with X-ray bright points (Moses et al. 1994). Cancellations of photospheric magnetic fields observed in relation to explosive events (Dere et al. 1991; Chae et al. 1998c) and bidirectional jets (Innes et al. 1997a) or chromospheric upflow events (Chae et al. 1998d) are strong indications of a generation process by reconnection. The spectroscopic signature of bidirectional jets seems to be a characteristic feature of explosive events.

Gallagher et al. (1999) also concluded, on the basis of transient-event observations in $\mathrm{O} v 62.3 \mathrm{~nm}$ and $\mathrm{He}$ I $58.4 \mathrm{~nm}$ with CDS, that magnetic reconnection produced the network events and powered the radiation, whereas shocks formed by acoustic waves were most likely responsible for the heating in inter-network regions. The energy release in a typical explosive event is estimated to be $1 \times 10^{16} \mathrm{~J}$ to $1 \times 10^{18} \mathrm{~J}$. It is, however, not clear if the plasma is heated to coronal temperatures during such events (Winebarger et al. 2002a, 2002b). In this context it has to be noted that explosive events observed in the N V $123.8 \mathrm{~nm}$ line formed at $1.8 \times 10^{5} \mathrm{~K}$ do not show a signature in the coronal line $\mathrm{Mg}$ X $62.5 \mathrm{~nm}$, but enhancements of the wings in a S II line (Teriaca et al. 2002a). The conclusion from this observation is that explosive events do not contribute significantly to the energy balance of the corona. The same conclusion was reached by Teriaca et al. (2002b, 2004).

Important questions in relation to explosive events are the variations of the electron density and temperature. The first density results of $n_{\mathrm{e}} \approx 7 \times 10^{10} \mathrm{~cm}^{-3}$ were obtained by Dere et al. (1991). Line-ratio studies later indicated that there is a density increase by a factor of about three and, in some cases, a significant electron temperature increase (Wilhelm et al. 1998c; Teriaca et al. 2001, 2002a; Mendoza-Torres et al. 2005). The small scale of the events makes such observations difficult, and more work is required with higher resolution spectrometers than are presently available. In addition, it has to be realised that the ionization equilibrium assumed may not be fully valid for these dynamic events. The average size is estimated to be $1.8 \mathrm{Mm}$ with a birth rate of $2500 \mathrm{~s}^{-1}$ over the entire Sun. Doyle et al. (2006b) reported sequences of explosive events at a rate of $\approx 0.3 /$ min near a coronal-hole boundary, and suggest repetitive reconnection triggered by kink waves in closed field-line regions.

Innes (2001) investigated quiet-Sun transition-region events in great detail using SUMER, TRACE, and MDI data. The strongest events were observed in a region with a complex magnetic field evolution. The Si IV profiles developed blue and red wings before the core brightened, indicating that plasma acceleration preceded compression or heating. Large density fluctuations accompanied the activity. There were indications of compressional waves with speeds of $\approx 30 \mathrm{~km} \mathrm{~s}^{-1}$.

The characteristics of quiet-Sun cell and network brightenings have been studied with CDS He I $58.4 \mathrm{~nm}$ and O v $62.9 \mathrm{~nm}$ observations by Harra et al. (2000). Network events are about an order of magnitude larger than cell events and last $\approx 150 \mathrm{~s}$ compared to $\approx 100 \mathrm{~s}$ for cells. The many similarities of the events lead to the suggestion that magnetic reconnection is operating in both regimes.

An unusual spectral behaviour of the $\mathrm{NV} 123.8 \mathrm{~nm}$ and $\mathrm{OV} 62.9 \mathrm{~nm}$ lines during an explosive event was observed by Doyle et al. (2006c). A strong red jet with $\approx 75 \mathrm{~km} \mathrm{~s}^{-1}$ in the $\mathrm{O} v$ line did not show up in the $\mathrm{N} v$ line, although the formation temperature of the latter is only slightly lower than that of $\mathrm{O} v$. The authors suggested that strong density gradients might be responsible for the effect. 
2.2.1.4 Blinkers With CDS, Harrison (1997) identified EUV brightenings exhibiting radiance enhancements by a factor of two to three in transition-region lines, and named them "blinkers". Blinker characteristics are: (a) an average duration of $13 \mathrm{~min}$, (b) a size $\approx 6 \mathrm{Mm} \times 6 \mathrm{Mm}$, and (c) no significant Doppler broadening. Events of this type were studied in more detail by Harrison et al. (1999).

Teriaca et al. (2001) measured the density-sensitive radiance ratio of O IV $L(140.1 \mathrm{~nm}) /$ $L(140.5 \mathrm{~nm})$ during a short-duration blinker and found no increase in the electron density, in contrast to their observations in explosive events (cf., Sect. 2.2.1.3). A poor correlation of blinker radiance variations in the chromosphere, transition region, and corona, observed in the He I $58.4 \mathrm{~nm}, \mathrm{OV} 62.9 \mathrm{~nm}$, and $\mathrm{Mg}$ IX $36.8 \mathrm{~nm}$ lines, respectively, was found by Brković et al. (2001). Peter and Brković (2003) studied blinkers in detail and found that the line width in O VI nearly reached thermal values at the maximum of the event. The lack of substantial nonthermal velocities and the missing signature of blinkers in the corona are used as arguments for a chromospheric driver of blinkers.

Explosive events simultaneously appear with blinkers at their edges. This result was obtained by Chae et al. (2000b) from CDS and SUMER observations. The authors concluded that magnetic reconnection is probably the cause of both events, but the magnetic field geometry is different in the sense that loops of the same size interact in the case of brightenings, whereas small and large loops change their topology during explosive events. Harrison et al. (2003a) found that explosive events and blinkers are not directly related. Whereas the strong outflow jets observed in explosive events point to magnetic reconnection as explanation, blinkers are more likely caused by density enhancements or filling-factor variations. Taking into account the result of Teriaca et al. (2001) that, in contrast to explosive events, blinker brightenings are not accompanied by electron density enhancements, filling-factor variations remain as the most likely option.

A different behaviour of the Doppler shifts of explosive events and blinkers was also deduced by Madjarska and Doyle (2003), who suggested a relationship to on-disk spicules. In comparisons of blinkers and explosive events, Brković and Peter $(2003,2004)$ confirmed that these events are independent phenomena. Blinkers were identified by O'Shea et al. (2005) at off-limb locations that showed macrospicule activity (cf., Sect. 2.2.1.1). With high-cadence EIT observations it was possible to compare the light curves of blinkers and macrospicules (Madjarska et al. 2006). Their similarity supports the suggestion that blinkers are the on-disk counterparts of macrospicules.

2.2.1.5 Microflares and Nanoflares These features are associated with impulsive dynamical events that produce emissions with energy releases just above of $10^{17} \mathrm{~J}$ near the limit of the detection capabilities of present-day instrumentation. The distribution of the energy released forms a power law with a slope of $\gamma \approx-1.8$ as determined by Aschwanden et al. (2000a). However, Parnell and Jupp (2000) found values of $|\gamma|$ greater than two, which would imply - if the trend continues to lower energies - that the smallest events dominate the total flare-generated energy (cf., Sect. 2.2.2.6). In C IV observations with UVSP on SMM, short- and long-lived microflares in the magnetic network were identified as impulsive heating events in small magnetic dipoles. The long-lived events correspond to X-ray bright points, and the short-lived ones may be related to explosive events (Porter et al. 1987).

\subsubsection{Large-Scale Solar Activity}

This section reviews the large-scale solar activity from the VUV data. Prominences or even quiescent prominences are seen as dynamic features, having narrower line width than quietSun areas. Eruptive prominences and filaments, sunspots and sunspot plumes, flares and CMEs are reviewed. 
2.2.2.1 Prominences The book on the nature of solar prominences by Tandberg-Hanssen (1995) and a recent review of prominence science with emphasis on SOHO contributions by Patsourakos and Vial (2002) cover this subject well and allow us to shorten this section substantially.

Prominences show a filamentary structure in $\mathrm{H} \mathrm{I} \mathrm{Ly} \alpha$ with many fine threads thinner than $2^{\prime \prime}$ (Bonnet et al. 1980). Using the UVSP instrument, the physical conditions of a quiescent prominence were studied in emission lines of the H I, He I, O IV, Si IV, and C IV spectra (Poland and Tandberg-Hanssen 1983). The varying appearance of the prominence with the formation temperatures of the lines led to the suggestion that many flux tubes of different temperatures were involved.

The He II $164 \mathrm{~nm}$ multiplet observed with the S082B instrument in a prominence was studied by Laming and Feldman (1993). They concluded that the prominence plasma can be described by an ionization equilibrium model, and derived a preliminary helium-tohydrogen abundance ratio of 0.1 .

The Ne VII $46.5 \mathrm{~nm}$ line (exhibiting a considerable limb brightening) shows absorption effects near prominences (Tousey et al. 1973). Continuum absorption of other spectral lines shortwards of the H I Lyman edge at $91.2 \mathrm{~nm}$ can also be observed, in prominences and filaments, but in other areas of the Sun as well (for instance, in quiet-Sun and coronal-hole regions) (Schmahl and Orrall 1979). The H I Lyman continuum is most likely responsible for this effect, but the He II Balmer continuum might also contribute to this absorption. The absorption has been studied and confirmed with CDS data by Kucera et al. (1998), who obtained a column density of $1 \times 10^{18} \mathrm{~cm}^{-2}$ of hydrogen in a prominence, and a filling factor greater than $\approx 0.3$. From studies of the Lyman continuum, Parenti et al. (2005a) obtained electron temperatures between $7500 \mathrm{~K}$ and $8300 \mathrm{~K}$ in a prominence. Ground-based observations and spectroscopic spacecraft data of the H I Lyman series and the He I lines at $53.7 \mathrm{~nm}$ and $58.4 \mathrm{~nm}$ obtained with SUMER showed a cool prominence body with $T_{\mathrm{e}}<1 \times 10^{4} \mathrm{~K}$, surrounded by hotter layers with temperatures of $\approx 6 \times 10^{4} \mathrm{~K}$. The corresponding nonthermal velocities are $\approx 4 \mathrm{~km} \mathrm{~s}^{-1}$ and $\approx 20 \mathrm{~km} \mathrm{~s}^{-1}$, respectively (de Boer et al. 1998; Dammasch et al. 2003).

The spectrum of the H I Lyman lines of several prominences was obtained by Heinzel et al. (2001a) and Stellmacher et al. (2003) with SUMER. The latter authors, observing with ground-based and space instrumentation, found that the extent of two prominences in emission lines with transition-region formation temperatures was larger than the main prominence body. The radiances and shapes of the lines varied substantially. In particular, no reversals in lines higher than $\operatorname{Ly} \alpha$ were found in one case, whereas, in other cases, all lines had rather strong reversals. Heinzel et al. (2001a) argued that, in the first case, the prominence-corona transition region is seen along the magnetic field and across in the second case.

EIT observations of jets in He II $30.4 \mathrm{~nm}$ prominences and MDI magnetograms led Wang $(1999,2001)$ to suggest that even quiescent prominences are of a dynamic nature, in which chromospheric material streams from one end of an arcade loop to the other with speeds of $\approx 30 \mathrm{~km} \mathrm{~s}^{-1}$. This is consistent with the findings that spectral lines formed at transitionregion temperature indicate high LOS velocities $\left(\approx 20 \mathrm{~km} \mathrm{~s}^{-1}\right)$ in prominences (Mein 1977; Wilhelm et al. 1997; Wilhelm et al. 2002a).

A spectral atlas of a prominence in the range from $80 \mathrm{~nm}$ to $160 \mathrm{~nm}$ was prepared by Parenti et al. (2004, 2005b). Preliminary results indicate that, contrary to the expectations, the prominence appears to contain hotter material at lower rather than at greater heights and, in addition, confirm earlier results (Feldman and Doschek 1977b; Mariska et al. 1979; Wilhelm et al. 1997) that the line widths in a prominence are narrower than in quiet-Sun areas. 
2.2.2.2 Filaments Filaments and prominences are two aspects of the same solar phenomenon. The only difference is that filaments are observed on the disk and prominences above the limb.

Chiuderi Drago et al. (2001) studied a filament with data from CDS and SUMER and compared them to microwave observations obtained with the Nobeyama radioheliograph and the Very Large Array (VLA). At short wavelengths, the H I Lyman continuum absorption suppresses the transition-region emission and thus makes the filament discernible in this range, but at wavelengths longer than the Lyman edge there is no difference between the radiance of a filament from that of its neighbourhood. A comparison of filament observations in $\mathrm{H} \alpha$, obtained with the Multichannel Subtractive Double Pass (MSDP) spectrograph of the Telescopio Heliografico para el Estudio del Magnetismo y de las Inestabilidades Solares (THEMIS), and EUV emission lines in the wavelength range from $52 \mathrm{~nm}$ to $63 \mathrm{~nm}$ seen with CDS revealed that the filament is much more extended in EUV than in $\mathrm{H} \alpha$ (Heinzel et al. 2001b). As an explanation the authors discussed H I Lyman continuum absorption, and concluded that the $\mathrm{H} \alpha$ filament is surrounded by cool material.

Models involving twisted magnetic fields in a dip configuration to support this material have recently been presented by Anzer and Heinzel (2006). A multi-wavelength study of a filament involving ground-based, SOHO and TRACE instruments indicated that relatively cool plasma extends to great altitudes reaching the $\mathrm{H} \alpha$ filament at $\approx 100 \mathrm{Mm}$ (Schmieder et al. 2004). Del Zanna et al. (2004) studied several quiescent filaments with CDS and SUMER and confirmed that the radiance in O v $62.3 \mathrm{~nm}$ is lower over the filament than in quiet-Sun regions. But they also found lower radiances in C III $97.7 \mathrm{~nm}$ and N III $99.2 \mathrm{~nm}$ above the H I Lyman edge.

Filament oscillations were studied by Okamoto et al. (2004), who found that EIT waves (cf., Sect. 2.1.4.5) caused the oscillations.

\subsubsection{Eruptive Prominences and Filaments A spectrum of an erupting prominences in} the wavelength range from $30 \mathrm{~nm}$ to $63 \mathrm{~nm}$ was observed by S082A (Widing et al. 1986). The eruption was associated with a large flare and was the only one seen during the Skylab mission that showed emission in O IV, O V and Ne VII, in addition to He I and He II.

An eruptive prominence was also studied by Fontenla and Poland (1989) using SMM observations. The plasma was heated to transition-region temperatures with almost no cool core left. No CME followed, but the electron density in the corona above the prominence was reduced. A flux-rope model for a CME following an erupting prominence was suggested by Yurchyshyn (2002), based on observations from the ground and SOHO. Such a model was discussed more than 20 years ago by Mouschovias and Poland (1978).

2.2.2.4 Active Regions Active regions are characterized by strong magnetic fields with high temporal variability. Studies in the Si VI and O VI emission lines with SMM showed frequent brightenings with durations of less than a minute in the brightest portions of active regions (Porter et al. 1984). In addition to strong loops between bipolar magnetic regions, weaker loops from one of the activity centres also connect to other areas outside the region (Tousey et al. 1973).

Many spectral studies of active regions were carried out in the past and significantly contributed to the identification of new spectra as reviewed by Wilhelm (2003). The spectra of loop structures in an active region, studied by Doyle et al. (1985a) with the help of Skylab data, indicated a rather constant electron density up to temperatures of several million kelvin. A recent spectrum of an active region observed by SERTS was presented by Thomas and Neupert (1994). Cook et al. (1983) performed a detailed study of localized emission enhancements with HRTS. 
Systematic observations of active regions before and during the SOHO and TRACE era were undertaken by SERTS and Yohkoh. Plasma temperatures of 2 MK to 3 MK were obtained with a steep decrease of the emission measure above this range (Schmelz et al. 1999). Taking into account microwave polarization observations, longitudinal magnetic fields of $3 \mathrm{mT}$ to $6 \mathrm{mT}$ were deduced for heights between $5 \mathrm{Mm}$ and $10 \mathrm{Mm}$ in the corona (Brosius et al. 1993, 1996). Compared to theoretical expectations for static, uniformly heated loops, TRACE observations indicated that many active-region loops have much higher radiances, and presumably higher electron densities (Lenz et al. 1999; Aschwanden et al. 2000b, 2001a). They are referred to as "overdense" loops, because the observed density is in excess of that predicted by scaling laws. Warren and Winebarger (2003) and Winebarger et al. (2003) confirmed the existence of overdense loops above the core of an active region with electron densities of more than $1 \times 10^{8} \mathrm{~cm}^{-3}$ (derived from density-sensitive line ratios of Si VIII and S X), and suggested that impulsive heating can account for the large densities. Electron densities of $5 \times 10^{9} \mathrm{~cm}^{-3}$ have been deduced from Si IX emission-line ratios measured by SERTS in the core of an active region (Keenan et al. 2003). Temperature variations seem to exist both from loop to loop and along a loop (Chae et al. 2000a).

Active regions are very dynamic at transition-region temperatures, but show less Doppler shift at coronal temperatures (Nicolas et al. 1982; Feldman et al. 1982; Kjeldseth-Moe and Brekke 1999; Spadaro et al. 2000; Brekke et al. 2000; Fredvik et al. 2002). In addition, rotational motions around the loop axes were found with speeds up to $50 \mathrm{~km} \mathrm{~s}^{-1}$. SERTS measured shifts of lines emitted by highly ionized iron from Fe X to Fe XVI. These measurements indicate a dynamic and turbulent corona in the active-region core, but also suggest a net upflow of the hot test material and a downflow of cooler material in the same region with relative speeds of about $15 \mathrm{~km} \mathrm{~s}^{-1}$ (Brosius et al. 1999). Much higher LOS velocities of up to $60 \mathrm{~km} \mathrm{~s}^{-1}$ were observed in cool active region loops with CDS (Brekke et al. 1997b). In a joint TRACE and SUMER study, Winebarger et al. (2002c) found significant red shifts in steady TRACE loops. Even if the revised Ne VIII rest wavelength of $77.0428 \mathrm{~nm}$ is taken into account (cf., Part I, Sect. 9.1), downflows of $\approx 10 \mathrm{~km} \mathrm{~s}^{-1}$ to $30 \mathrm{~km} \mathrm{~s}^{-1}$ were observed.

An active-region loop system observed by EIT on 30 August 1996, was analysed with a dynamic stereoscopy method in order to construct the 3-D configuration of the loop system (Aschwanden et al. 1999b, 2000c). A potential magnetic field model computed from an MDI magnetogram reasonably traced the EIT loops. The loop showed, in general, no thickness variations.

Coronal plasma flows and magnetic fields in solar active regions were studied by Marsch et al. (2004), using combined observations from SOHO and magnetograms from the KPNSO. These data were used to construct the coronal magnetic field by force-free field extrapolation. The combined dataset was used to study large-scale plasma flows in three active regions associated with sunspots. The Doppler shift pattern was found to be closely linked with the field topology, and several regions of up and down flows and strong velocity shear were identified.

As a special form of EUV emission from the active transition region and low corona, the so-called "moss", has been discovered with the help of TRACE observations (Schrijver et al. 1999; Berger et al. 1999). The same feature was identified in soft X-ray images of NIXT (Peres et al. 1994). The moss appears to be a mixture of dark absorbing material and hot coronal plasma (at $\approx 1 \mathrm{MK}$ ) near the legs of even hotter coronal loops (Martens et al. 2000). An example is shown in Fig. 11.

2.2.2.5 Sunspots and Sunspot Plumes The plasma above sunspots is cooler than in its surroundings. Doppler shifts obtained with the S082B spectrograph indicated subsonic and 
Fig. 11 Moss region, observed by TRACE in the $17.1 \mathrm{~nm}$ passband on 6 June 1999. The square box of $19^{\prime \prime} \times 19^{\prime \prime}$ contains bright moss structures with a brightness distribution given in the histogram inset.

Active-region loops can be seen in the neighbourhood (Martens et al. 2000)

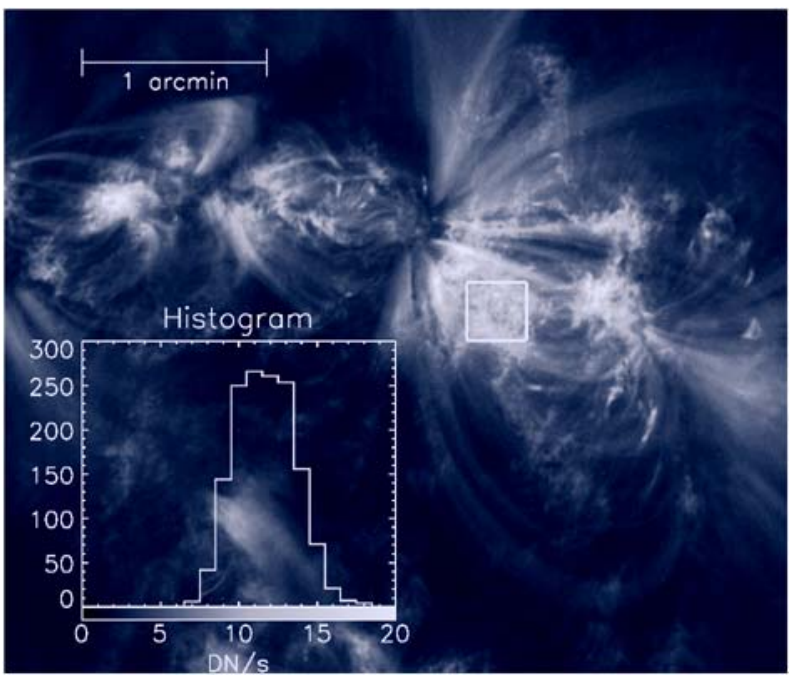

supersonic flows along the same LOS (Nicolas et al. 1982). A Doppler-shifted Mg IX line was observed by SERTS over a large sunspot indicating an outflow speed of $\approx 14 \mathrm{~km} \mathrm{~s}^{-1}$ relative to the surrounding plasma in the low corona (Neupert et al. 1992). Doppler-width measurements of the C IV $154.8 \mathrm{~nm}$ line over sunspot umbrae yielded narrower profiles than for quiet-Sun regions (Gurman et al. 1982; Athay et al. 1983).

Sunspots are, in general, not very prominent features in the VUV spectral range, but sunspot plumes are. The latter are relatively cool structures guided by magnetic fields with quite unusual spectra in the surrounding hot active regions (Foukal et al. 1974; Noyes et al. 1985; Curdt et al. 2001). Sunspot plumes are oscillating both in radiance and in velocity with periods of about 3 min (Gurman et al. 1982; Lites 1992; Maltby et al. 2001; Fludra 2001; Brynildsen et al. 2002). These oscillations are much more prominent in the blue wing of the emission lines than in the red wing (Brynildsen et al. 2003). Umbral oscillations with periods between $112 \mathrm{~s}$ and $185 \mathrm{~s}$ could be seen with CDS and TRACE inside and outside of sunspot plumes; in some cases different frequencies have been observed cospatially and simultaneously (O'Shea et al. 2002).

Note that the sunspot plumes are totally different from plumes in coronal holes. Many $\mathrm{H}_{2}$ lines are enhanced in specific regions of the sunspot umbra, excited by fluorescence from $\mathrm{H} \mathrm{I}$ Ly $\alpha$ (Jordan et al. 1978; Schühle et al. 1999). Complicated flow geometries, including dual flows with supersonic speeds, have been detected in the sunspot transition region (Brynildsen et al. 2001, 2004; Marsch et al. 2004). Dramatic changes in the flow velocity pattern during the disappearance of a sunspot plume were observed by Brosius (2005) with CDS, EIT and MDI. With additional radio observations from VLA, Brosius and White (2004) concluded that the radio signal of the umbra was dominated by the thermal gyroemission from the sunspot plume. With the help of the density-sensitive ratios of the $\mathrm{O} v$ lines near $76.0 \mathrm{~nm}$, Doyle and Madjarska (2003) deduced electron densities in sunspot plumes of $9 \times 10^{9} \mathrm{~cm}^{-3}$, confirming earlier Skylab measurements (Doyle et al. 1985b). In the quiet-Sun region surrounding the spot, the electron density was more than a factor of two higher. Brosius and Landi (2005) determined the DEM of a sunspot plume with a broad peak below $1 \mathrm{Mm}$.

2.2.2.6 Flares Solar flares (sometimes called chromospheric eruptions) produce the hottest solar plasmas which are available for spectroscopic investigations. Temperatures 
above $10 \mathrm{MK}$ are quite common in strong flares and lead to spectra with lines of highly ionized species extending into the X-ray range. Flare spectral observations from Skylab instruments S082A and S082B and from SUMER can be found, for instance, in Doschek et al. (1975b), Dere (1978), Cohen et al. (1978), and Feldman et al. (2000b). A class M2 flare was observed by the S082A spectrograph in the He II lines at $25.6 \mathrm{~nm}$ and $30.4 \mathrm{~nm}$, and Fe XV at $28.4 \mathrm{~nm}$ (Tousey et al. 1977). Large Doppler shifts towards shorter wavelengths were seen in He II and Fe XV corresponding to LOS velocities of $300 \mathrm{~km} \mathrm{~s}^{-1}$ to $600 \mathrm{~km} \mathrm{~s}^{-1}$.

Using X-ray spectra emitted by intense (X-class) and moderately intense (M-class) flares recorded by the high-resolution Naval Research Laboratory (NRL) Solar Flare Xrays (SOLFLEX) crystal spectrometers on the satellite P78-1 (launched in 1979), a series of detailed studies of temperatures and mass motions in flares were performed (Doschek et al. 1979, 1980, 1981a; Feldman et al. 1980a, 1980b). According to these studies, typical turbulence velocities are of the order of $130 \mathrm{~km} \mathrm{~s}^{-1}$ during the rise phase of a flare, and $60 \mathrm{~km} \mathrm{~s}^{-1}$ during the decay phase. At the onset, blue shifts of the order of $150 \mathrm{~km} \mathrm{~s}^{-1}$ to $300 \mathrm{~km} \mathrm{~s}^{-1}$ were also observed, occasionally even $500 \mathrm{~km} \mathrm{~s}^{-1}$.

Antonucci et al. (1982) studied soft X-ray emission observed with SMM, and also found turbulent velocities of more than $100 \mathrm{~km} \mathrm{~s}^{-1}$ and upflows with speeds up to $400 \mathrm{~km} \mathrm{~s}^{-1}$. Similar results were obtained from the Bragg Crystal Spectrometer (BCS) on Yohkoh (Mariska 1994). Occasionally, even higher LOS velocities were seen in a limb flare of class M7.6 (Innes et al. 2001). Density diagnostics provided values of up to $3 \times 10^{12} \mathrm{~cm}^{-3}$ for a flare occurring on low-lying preexisting loops (Doschek et al. 1981b; Cheng et al. 1982). Using Fe XXI to Fe XXII line ratios in the X-ray range $(0.8 \mathrm{~nm}$ to $1 \mathrm{~nm})$, Phillips et al. (1996) reported electron densities that reached values of $1 \times 10^{13} \mathrm{~cm}^{-3}$ near the flare maximum.

Multi-wavelength observations from SMM and from the ground were used by MacNeice et al. (1985) to study an M1.4 flare with upflows exceeding $200 \mathrm{~km} \mathrm{~s}^{-1}$ in Ca XIX. New magnetic flux emerged at the flare site, and a filament erupted two minutes before the flare. Major flares dissipate an energy of more than $1 \times 10^{27} \mathrm{~J}$, such as the X20 flare on 2 April 2001 (probably the largest flare on record), down to $\approx 1 \times 10^{18} \mathrm{~J}$, if microflares are included. The dependence of the flare occurrence rate on the flare energy has been a subject of research for many years (cf., Feldman et al. 1997; Benz and Krucker 2002). Power-law indices between $|\gamma| \approx 1.9$ and $\approx 2.6$ have been reported, and thus the limit of two, critical for the contribution of most of the energy by small events, is still not adequately constrained (see also Sect. 2.2.1.5).

One of the best-observed flares, the "Bastille Day Flare" of 1998, was described by Aulanier et al. (2000). During the impulsive phase of a X3/3b class flare, Brekke et al. (1996) observed with SOLSTICE irradiance increases in the resonance lines of Si IV and C IV by a factor of about 12. A fractional flare size of $0.08 \%$ of the solar disk was estimated, using $\mathrm{H} \alpha$ images of the Udaipur Solar Observatory, and allowed the authors to estimate a radiance increase in these lines of $\approx 1.5 \times 10^{4}$. During the X5.3/3b flare on 25 August 2001, SUMER was observing in a Sun-as-a-star mode (Lemaire et al. 1998, 2004). The irradiance increases in the $\mathrm{H}$ I continuum near the Lyman edge and the $\mathrm{C}$ II multiplet at $90.4 \mathrm{~nm}$ could be translated into radiance increases with respect to quiet-Sun levels by determining the flare area from a ground-based $\mathrm{H} \alpha$ image. Taking into account the sampling time, an increase of $1 \times 10^{4}$ was estimated.

The helium-like ions are of special interest, because relativistic calculations can be performed for this two-electron configuration. The first identifications of lines from helium-like ions in solar spectra were reported by Gabriel and Jordan (1969a, 1969b). Many of the $2 \mathrm{~s}^{3} \mathrm{~S}$ $2 \mathrm{p}^{3} \mathrm{P}$ transitions in $\mathrm{Ne}^{8+}, \mathrm{Na}^{9+}, \mathrm{Mg}^{10+}$, and $\mathrm{Si}^{12+}$ have recently been observed in solar flare plasmas, in which also the wavelength of the $\mathrm{NaX}$ line at $111.177 \mathrm{~nm}$ could be measured 
for the first time (Curdt et al. 2000). The corresponding O VII line had been tentatively identified at $162.4 \mathrm{~nm}$ in an early eclipse spectrum by Gabriel et al. (1971). This identification was then confirmed using Skylab spectra by Feldman and Doschek (1977c).

Chromospheric evaporation by precipitation of nonthermal electrons is thought to be an important process during a solar flare (cf., Fisher et al. 1985). However, the high upflow speeds expected from such a model have not been found in spectroscopic observations. Warren and Doschek (2005) and Doschek and Warren (2005) suggested a multithread concept of the flare loop assembly with staggered heating events to reconcile the observations with the model simulations.

In a large post-flare arcade, a number of dark, downward-moving flows could be seen in $19.5 \mathrm{~nm}$ images of TRACE (Dobrzycka et al. 2003). SUMER spectra of lines with formation temperatures between $2 \times 10^{4} \mathrm{~K}$ and $10 \mathrm{MK}$ show that the flows are dark in the hottest line Fe XXI, but not in the cooler one (Innes et al. 2003). Since there was no absorption in the H I Lyman continuum, the conclusion was reached that the dark flows correspond to voids. This confirms a suggestion made by McKenzie and Hudson (1999) in relation to similar plasma features seen with SXT.

Reconnection inflow with an apparent speed of $5 \mathrm{~km} \mathrm{~s}^{-1}$ and a plasmoid ejection could be detected during a flare by SXT and EIT (Yokoyama et al. 2001).

Observations of spectral lines with formation temperatures between $2 \times 10^{4} \mathrm{~K}$ and 7.9 $\mathrm{MK}$ at a height of $70 \mathrm{Mm}$ above the solar limb during a sequence of flares indicated that the flaring plasma is heated in situ from $\approx 1 \mathrm{MK}$ to $\approx 10 \mathrm{MK}$, possibly by compression of the coronal plasma (Feldman et al. 2004). The conclusion is based on the temporal development of the LOS radiances.

2.2.2.7 Coronal Mass Ejections The discovery and many detailed CME observations were achieved with white-light coronagraphs (see, e.g., Munro et al. 1979), which will not be addressed any further here. The VUV observations described in this review relate to the signatures of CMEs at relatively low altitudes, and are thus of relevance for their onsets. CMEs with interesting helical structures were seen by LASCO, UVCS, and EIT (Dere et al. 1999; Ciaravella et al. 2000) and interpreted as helical magnetic flux ropes. A strong Earthdirected CME was observed by EIT in May 1997 (Thompson et al. 1998). In five cases, coronal EUV dimmings occurred in association with CMEs (Harrison et al. 2003b). Spectral observations with CDS in many emission lines led to the conclusion that the dimming is caused by mass loss of the corona and not by temperature variations. The estimated mass loss $\left(\approx 1 \times 10^{11} \mathrm{~kg}\right)$ is a typical value for CMEs. Coronal dimmings during CMEs were also described by Zarro et al. (1999), Thompson et al. (2000) as well as Harrison and Lyons (2000). From data obtained by the SMM coronagraph, Harrison (1995) concluded that flares and CMEs are closely related, but do not drive one another.

Sterling et al. (2000) studied a halo CME that originated from near-disk-centre locations and showed a "sigmoid" in SXT. The S-shaped structures along neutral lines were most prominent at temperatures of $2.4 \mathrm{MK}$, but much weaker or absent in EIT channels at lower temperatures. During the flare, a narrow sigmoid brightened up in EIT channels, and postflare loops normal to the neutral line developed. The temporal relationship between CMEs and flares was also studied by Zhang et al. (2001b), with the result that the initial phase of CMEs occurs before the onset of the associated flare which coincides, however, with the acceleration phase of the CME.

The relation between flares and CMEs was also studied by Zhang and Golub (2003) with the help of high-cadence TRACE observations. It was found that fast CMEs are associated with two-ribbon brightenings during the flare, slow CMEs sometimes show tubular emission. Different magnetic field configurations are suggested for these events. 
Joint observations of SXT (Tsuneta et al. 1991), EIT, SUMER, and the Mirror Coronagraph for Argentina (MICA) led to the identification of a flare and CME onset with expansion speeds of up to $650 \mathrm{~km} \mathrm{~s}^{-1}$ of the $10 \mathrm{MK}$ plasma (Innes et al. 2001). A shock wave is thought to heat and accelerate the loop structure. Three CMEs associated with X-class flares have been observed with UVCS (Raymond et al. 2003). In contrast to other CMEs, no cool prominence material could be seen in these events, but the O VI lines at $103.2 \mathrm{~nm}$ and $103.7 \mathrm{~nm}$ showed a splitting indicative of LOS velocities of $\pm 800 \mathrm{~km} \mathrm{~s}^{-1}$.

A catalogue of the EIT transient waves and the associated type II radio bursts in 1997 was compiled by Klassen et al. (2000). It could not be unambiguously shown that both wave phenomena are different signatures of the same disturbance, although there are such indications.

\subsection{Elemental Abundances}

Since the early days of solar spectroscopy, the solar-system composition has been considered to be among the most fundamental set of parameters in astrophysics. Abundances derived by spectroscopic means may have important uncertainties in the assumptions regarding the plasma properties and/or the atomic parameters. Traditionally, elemental abundances are determined relative to hydrogen. Such studies could be carried out by comparing radiance ratios of lines from selected ions with an $\mathrm{H}$ I line or with the free-free continuum. Since some spectrometers operate at wavelength ranges where $\mathrm{H}$ I lines do not appear or the free-free continuum is too faint to be observed, other elements such as $\mathrm{O}, \mathrm{Si}$ and Fe are often used as proxies. The methods of determining the elemental abundances in the solar atmosphere were reviewed by Mason (1995) with emphasis on the atomic physics parameters assumed in the calculations. A summary of solar abundance measurement methods is contained in a rapporteur paper by von Steiger et al. (2001).

\subsubsection{Photospheric Composition}

Russell (1929) made the first quantitative analysis of the chemical composition of the solar atmosphere. Using eye estimates of the solar line radiances, he derived the abundances of 56 elements. Russell also showed that the solar atmosphere and, finally, the universe were essentially made of hydrogen. Using better observations and techniques, Unsöld (1948) obtained abundance results for 25 elements, while noting that his results were not much different from Russell's. He concluded that this was not surprising because Russell had an "unvergleichliches spektroskopisches Fingerspitzengefühl" (an incomparable spectroscopic flair).

Since then a significant body of data regarding photospheric composition values derived by spectroscopic means was accumulated. The composition of nonvolatile elements in meteorites designated as carbonaceous Ivuna (CI) chondrites were found to have a composition that is identical to that of the solar photosphere (Goldschmidt 1937). Because of the very accurate laboratory measurements with $2 \%$ relative standard uncertainties of the composition of CI chondrites, their values are often used as checks on photospheric abundances of nonvolatile elements derived by spectroscopic means.

The 15 most abundant elements in the solar photosphere can be divided into two distinct groups. The first group includes the nonvolatile elements $\mathrm{Na}, \mathrm{Mg}, \mathrm{Al}, \mathrm{Si}, \mathrm{Ca}, \mathrm{Fe}$, and $\mathrm{Ni}$. The second group consists of volatile elements that can further be divided into two subgroups. The first one includes the volatile elements $\mathrm{H}, \mathrm{N}, \mathrm{C}, \mathrm{O}$, and $\mathrm{S}$ and the second includes the noble gases $\mathrm{He}, \mathrm{Ne}$ and $\mathrm{Ar}$. Since the volatile elements were not faithfully retained in meteoritic 
material, their abundance needs to be obtained by other means. For the volatile elements $\mathrm{H}$, $\mathrm{C}, \mathrm{N}, \mathrm{O}$, and $\mathrm{S}$, the photosphere is the best source of their abundances. Unfortunately, these values are not yet well established and undergo occasional modifications.

A case in point is the abundance of oxygen, $A(\mathrm{O})$, relative to hydrogen which, when being measured on the logarithmic scale ${ }^{8}$ defined by $\lg A(\mathrm{H})=12$, i.e., the number of oxygen atoms per $1 \times 10^{12}$ atoms of hydrogen, was found to have changed during the last 16 years from $\lg A(\mathrm{O})=8.93$ (Anders and Grevesse 1989) to $8.83 \pm 0.05$ (Grevesse and Sauval 1998), then to $8.69 \pm 0.05$ (Allende Prieto et al. 2001), and most recently to $8.66 \pm 0.05$ (Asplund et al. 2004). Relative uncertainties in the abundances of the volatile elements are of the order of $15 \%$. Much of the recent research on the composition of the solar photosphere is summarized in these references and in review articles by Anders and Grevesse (1989), Grevesse et al. (1996, 2005). Since noble gases are not represented at all in the solar photospheric spectra their abundances had to be derived from other measurements.

\subsubsection{Composition of the Corona}

The composition of the solar photosphere is believed to be well defined, and it does not to vary with solar surface location or from one particular solar feature to another. In the early years of space exploration, it was even assumed that the solar composition everywhere in the atmosphere was identical to that of the photosphere. Mariska (1980) studied the relative chemical abundances at various heights above the limb. The spectra were obtained by the Skylab S082B spectrograph above quiet-Sun regions, a coronal hole, an active region, and a prominence and did not indicate significant differences in these regions.

Yet, during the last 25 years, the studies of the solar atmosphere proceeded from first recognition of systematic differences between the composition of the corona and the photosphere to solid evidence for spatial and temporal variations in the composition of the different coronal features. Early indications of anomalous coronal composition were seen in low-speed solar wind and energetic-particle data, in which elements with a first-ionization potential (FIP) $<10 \mathrm{eV}$ (low FIP) were overabundant by approximately a factor of four relative to those with a FIP $>11 \mathrm{eV}$ (high FIP) (Hovestadt 1974; Geiss 1982; Breneman and Stone 1985). This suggested that a separation of ions from neutrals at the base of the atmosphere could be responsible (Geiss 1982).

Using published results on the abundances obtained by ultraviolet spectroscopy of various regions in the solar atmosphere, as well as the extensive data sets on the abundances of the solar wind and solar energetic particles measured in situ, Meyer (1985) succeeded in determining the difference in the composition of the photosphere and the upper solar atmosphere. He showed that there is an underabundance of high-FIP relative to low-FIP elements by a factor of four to six in the corona as compared to the standard local galactic or photospheric composition. In a series of later accounts (e.g., Meyer 1996), he changed his view and concluded that the low-FIP elements were overabundant relative to hydrogen by a factor of four or five, while the high-FIP elements retained nearly photospheric abundances. Feldman (1998) concluded that, compared with the photosphere, elements with FIP $\leq 10 \mathrm{eV}$ were overabundant by about a factor of four relative to elements with FIP $>10 \mathrm{eV}$. In the following years, a complex pattern in the modification of solar atmospheric plasma compositions began to emerge.

\footnotetext{
${ }^{8}$ With the common definition: $\lg x \equiv \log _{10} x$.
} 
The first three noble gases in the periodic table of elements have the highest ionization potentials among all elements. Due to the very high energies of their first excited levels, no absorption lines from $\mathrm{He}$ I, Ne I, and Ar I were observed in the photospheric spectrum. As a result, their photospheric abundance has never been established by a direct measurement. With spectra from Skylab and CDS, Widing and Feldman (1989), Feldman and Widing (1990), and Young et al. (1997) determined the photospheric abundances of neon and argon from their emission lines.

The first attempt to determine directly the helium abundance in the solar corona was made by Gabriel et al. (1995). Using data from the Coronal Helium Abundance Spacelab Experiment (CHASE) aboard the Spacelab II mission, the authors measured the radiance ratio between the $121.6 \mathrm{~nm} \mathrm{HI}$ and the $30.4 \mathrm{~nm} \mathrm{He} \mathrm{II}{ }^{9}$ resonance lines, and found that the helium fraction by mass is $Y=0.23 \pm 0.03$. Feldman (1998) used the radiance ratio between the $108.5 \mathrm{~nm} \mathrm{He}$ II Ba $\gamma$ line and the $103.1 \mathrm{~nm}$ O VI resonance line, as recorded by SUMER from a quiet coronal equatorial region, to derive the coronal helium abundance. Recently, Laming and Feldman (2001) repeated the measurement, using improved atomic data and a modified oxygen photospheric abundance value. The result was a mass fraction of $Y=0.13 \pm 0.02$.

In 2001 the Rentgenovsky Spektrometrs Izognutymi Kristalami (RESIK) instrument, consisting of four X-ray spectrometers covering the range from $0.33 \mathrm{~nm}$ to $0.61 \mathrm{~nm}$, was launched on the Russian Coronas-F mission (Sylwester et al. 2005). Using coronal He-like argon lines to free-free continuum radiance ratios emitted by a number of flares, Phillips et al. (2003) derived an absolute value for the argon abundance in the Sun. Landi et al. (2007) obtained the absolute neon abundance in the Sun from SUMER-recorded radiance ratios of a $125 \mathrm{~nm}$ He-like neon line to the free-free continuum.

To simplify discussions on elemental abundances the term FIP bias-the ratio between and low-FIP and high-FIP elements in the corona to the expected ratio in the photosphereis commonly used. The most abundant among the low-FIP elements are $\mathrm{Mg}, \mathrm{Si}, \mathrm{Ca}$, and $\mathrm{Fe}$, whereas $\mathrm{H}, \mathrm{He}, \mathrm{C}, \mathrm{N}, \mathrm{O}, \mathrm{Ne}, \mathrm{S}$, and Ar are abundant high-FIP elements. Feldman and Widing (1993), Young and Mason (1998), and Laming et al. (1995) measured the elemental abundances in the transition region of the quiet Sun and in coronal holes. A comparison between the low-FIP Mg and the high-FIP Ne abundance ratios from CDS in network and inter-network areas of the transition region showed only a weak FIP effect, which was not very dependent on the phase of the solar cycle (Young 2005). Doschek et al. (1998b) studied the $\mathrm{Si} / \mathrm{Ne}$ abundance ratio in inter-plume regions and found indications that this ratio was increased over its photospheric value by about a factor of two. Young et al. (1999) and Wilhelm and Bodmer (1998) studied the composition in polar plume using CDS and SUMER spectra. According to these studies, the magnesium/neon abundance ratio is enhanced by a factor of 1.5 to 3 in plumes with respect to the inter-plume regions.

The elemental compositions in the quiet corona along solar streamers and in a coronal hole were measured by many investigators (Raymond et al. 1997; Feldman et al. 1998; Warren 1999; Widing et al. 2005; Landi et al. 2006). Coordinated observations of streamers by CDS and UVCS showed photospheric abundances at low altitudes, whereas a depletion with respect to the photospheric values of several elements was noted at greater heights (Parenti et al. 2000).

Although a large number of composition measurements of active regions and flares were done with spectra in X rays, some used observations in the VUV from Skylab, SUMER and

\footnotetext{
${ }^{9}$ In Part I, Sect. 8.4.1 it should read He I instead of H II.
} 
CDS. With CDS, Young and Mason (1997) observed a FIP bias of 8.1 in a spiky structure lying at the edge of a newly emerged active region, which showed nearly photospheric abundances. Dwivedi et al. (1999) derived from SUMER observations in loop-like structures of an active region above the limb enrichment values of 8 to 16. Using EUV spectra of an active region observed off the solar disk by the SUMER spectrograph, Dwivedi et al. (2004) found that the relative $\mathrm{Mg} / \mathrm{Ne}$ abundance is highly variable in the complex, cool core of the active region, strongly correlated with line radiance and magnetic structures. $\mathrm{Mg}$ abundance enhancements relative to Ne reach up to a factor of 8.8. The analysis of the diffuse corona seemed to suggest that in off-limb active region plasma, the FIP bias inside the low-FIP class is higher for the element K. Also, some dependence of the FIP bias with height was suggested. FIP bias is still an open and more work is required. Widing and Feldman (1995) studied the $\mathrm{O} / \mathrm{Ne}$ and $\mathrm{Mg} / \mathrm{Ne}$ abundance ratios in a small sample of active regions observed by Skylab. They found that when active regions are observed with sufficient spatial resolution, the measured FIP-bias enrichment could significantly exceed a factor of four. Widing and Feldman (2001) reported measurements of elemental abundances in a number of active regions from the time they emerged over a week until ten days later. When first being measured, the abundances were photospheric. However, from shortly after and for the duration of the observations the abundance ratio continued to change until it reached a factor of $\approx 9$ after five to six days. For review papers describing the recent findings that relate to the composition of the solar atmosphere see Feldman (1992), Saba (1995), Fludra et al. (1999b), Feldman and Laming (2000), and Feldman and Widing (2003).

Ko et al. (2006) used in situ data from the Solar Wind Ion Composition Spectrometer (SWICS) on board the Advanced Composition Explorer (ACE) spacecraft at $\approx 1$ ua in combination with spectroscopic data from UVCS measured at $1.64 R_{\odot}$ to investigate the coronal origin of the solar wind. The analysis they presented supports the idea that a possible source of the slow solar wind is in the boundary region between coronal holes and active regions. Aided by data from UVCS, Vásquez and Raymond (2005) used semi-empirical models for the proton-electron global corona and, on the basis of empirical constraints on oxygen ions, they developed coronal oxygen models suitable for solar minimum conditions.

An interesting and new method of studying the iron abundance in the corona relative to its photospheric value has been developed by Aschwanden et al. (2003b). Cooling times of coronal loops measured with the TRACE $17.1 \mathrm{~nm}$ and $19.5 \mathrm{~nm}$ channels resulted, under the assumption that the cooling is predominantly controlled by radiation from iron ions, in enhancement factors of $\approx 4$.

All these observations of the solar atmosphere indicate: (a) Its composition appears to be modified only in plasmas confined in magnetic structures with a long lifetime. (b) In the corona over quiet regions, the average values of the FIP bias are three to four, whereas in open magnetic field regions (except in coronal plumes) the FIP bias is close to unity. (c) The magnitude of the FIP bias is time dependent; in old active regions values as high as 16 were measured. (d) FIP bias modification times are measured in hours or days and not in seconds or minutes.

The origin of the solar wind most likely occurs in fairly low-lying magnetically confined coronal structures. Thus, an understanding of the enrichment process, eventual disintegration of the structures, and the release of the enriched plasma into the solar wind may provide both insight and constraints on solar wind models. 


\section{Some Theoretical and Model Considerations}

In the previous sections, we have given a comprehensive account of the observations of the Sun and its corona in ultraviolet wavelengths from space. A very detailed and complex picture has emerged, in particular of the corona and its dynamic lower layer, the transition region, revealing continuous activity of the magnetic field on all scales resolved. However, even after many years of SOHO and TRACE observations, important questions concerning the workings of the solar corona and the origin of the solar wind remain open. As an introduction to this more theoretically oriented chapter, we list some of these outstanding questions:

(1) What heats the two types of corona, which are either magnetically closed, with dense plasma in magnetic confinement, or open with dilute plasma escaping freely on magnetic field lines?

(2) What determines the solar wind mass flux? Is it the plasma properties of the source region in the upper chromosphere or the temperature conditions in the corona?

(3) Is the slow solar wind mainly a boundary-layer phenomenon, or does it originate everywhere from transient openings of loops and streamers, thus releasing plasmoids like droplets from a leaking water tap?

(4) How well do we (and ought to) understand the transport phenomena in the weakly collisional but strongly microturbulent corona?

(6) What is the rôle of kinetic plasma waves in coronal heating?

In the recent past, unambiguous evidence for low-frequency waves and oscillations in coronal magnetic flux tubes and loops has been found by means of remote-sensing with SUMER and TRACE (see, e.g., the reviews by Aschwanden et al. 2001b; Wang 2004). Also, considerable progress has been made in characterizing the coronal plasma state and determining the radial profiles of key parameters, e.g., in the polar coronal holes. This success is largely due to the unprecedented and concerted effort of the SOHO and TRACE instruments. We consider and discuss in some detail some of the most salient points here, which are the physics of the magnetic transition region, coronal plasma confinement and release, heating of coronal holes, and initial acceleration of the solar wind, realizing that we have to leave out many important aspects of the physics of the solar atmosphere.

\subsection{Global Structure of the Coronal Magnetic Field}

Undoubtedly, the magnetic field is the main player in and major agent of the plasma processes occurring in the Sun's atmosphere, and especially in the low- $\beta$ corona. As the previous sections illustrated, the coronal VUV- and X-ray emissions are highly variable and nonuniform, and thus reflect the complex structure and activity of the coronal magnetic field confining the plasma. Unfortunately, a direct measurement of the magnetic field vector in the corona, though being in principle possible, has hardly been made and is far from being done routinely. In contrast, in photosphere and chromosphere the magnetic field vector has routinely been measured by means of full Stokes polarimetry, or its single components by the simple Zeeman effect, yet it is only very recently that the magnetic vector at the coronal base was measured (see Solanki et al. 2003) directly. This led to the detection of a current sheet and revealed the topological changes of the field with height in the transition region.

For the corona, we have to rely on models of the magnetic field, usually obtained by extrapolation through potential-field, force-free, or numerical magnetohydrodynamics (MHD) methods, starting from the photospheric bottom to the corona. For a modern description of 


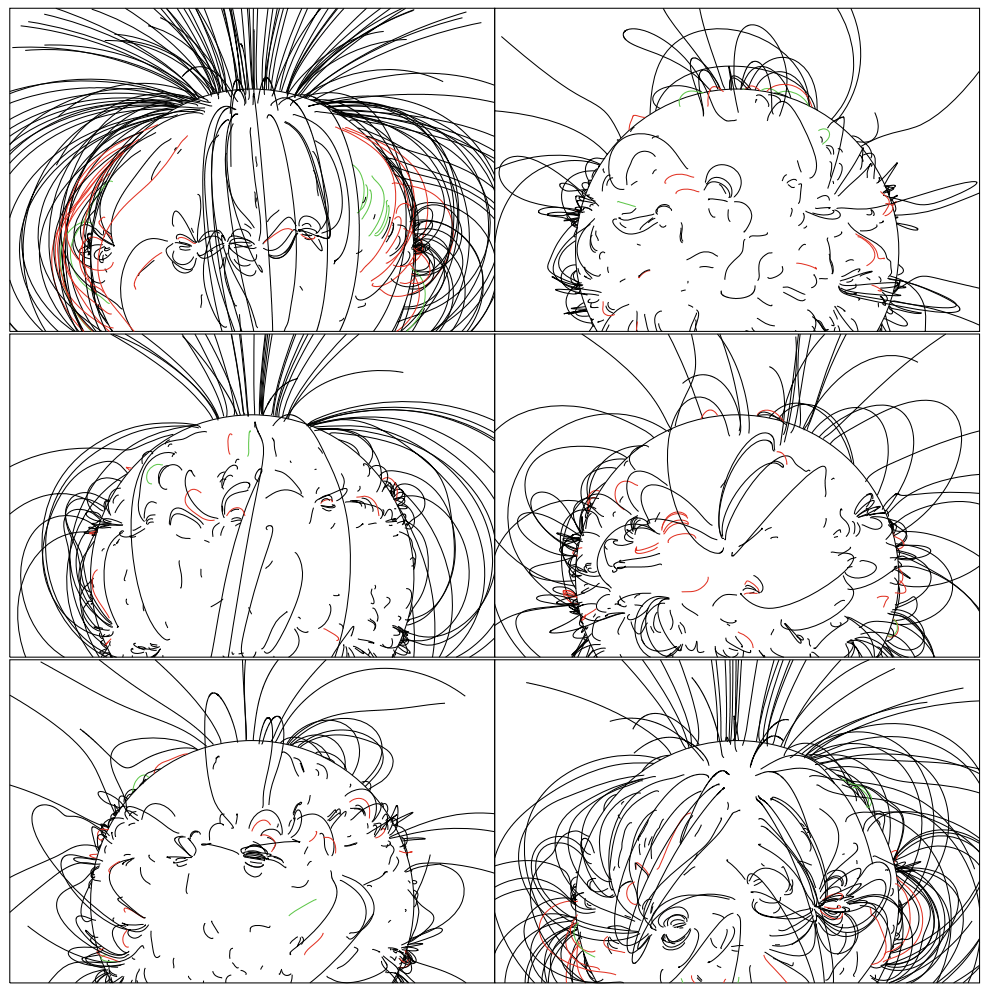

Fig. 12 Potential-field models of the coronal magnetic field for six different phases of the solar cycle (minimum: top left; maximum: top right) after Schrijver and Aschwanden (2002). Note the varying magnetic field topology, indicating closed loop-like structures (and streamers) and open funnel-like or ray-type structures (and polar plumes) in coronal holes as the main magnetic building blocks. Loops with expansion factors of less than two are shown in green, in red those with below four, and in black all others

these methods and their limitations see, e.g., Wiegelmann and Neukirch (2002). Figure 12 shows an example of the potential-field model of the magnetic field of the solar corona for six different phases of the solar cycle (Schrijver and Aschwanden 2002). The field may become obvious through plasma emission or remain obscure because of the lack of plasma.

In this way, modern imagers in X rays on Yohkoh, or in the VUV on SOHO and TRACE have provided us with detailed "images" of the coronal field, which was made visible through the emission of plasma confined therein. These space observatories have yielded a wealth of coronal images and have provided diagnostic information on the plasma state, thus enabling many deep insights into the physical processes, the morphology and activity of the solar corona. The new picture arising from all these observations is that the corona is a restless, intricate and magnetically coupled system. This "new solar corona" was reviewed by Aschwanden et al. (2001b).

Facing complex plasma and field dynamics in association with a wide range in spatial and temporal scales constitutes the main challenge in the physics of the solar corona. Typically, coronal images reveal a multitude of bright closed loops (on all scales down to $1^{\prime \prime}$ and even less), in coronagraph pictures the conspicuous streamers, and in striking contrast dark voids that correspond to coronal funnels and coronal holes. The emissions from the upper chromosphere (discussed in Sect. 2.1.2.2) and lower transition region (at temperatures be- 
Fig. 13 The solar transition region as seen in the VUV emission line of C III (after Peter 2001). About a quarter of the Sun is shown. Various structures are visible in this emission pattern, with the network cells being the most conspicuous. One cell is blown up in the insert at the top left, where small loops bridging the network lane become apparent. A huge prominence is visible over the left limb

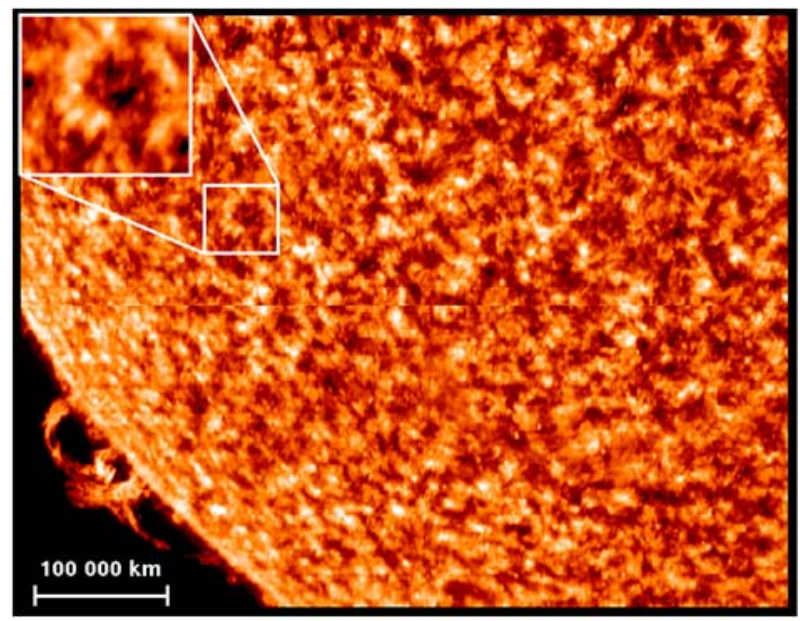

low about $10^{5} \mathrm{~K}$ ) exhibit the magnetic network (see Sect. 2.1.3.3) with typical cell sizes of $20 \mathrm{Mm}$ to $30 \mathrm{Mm}$, which seem to be related to myriad small loops. All this is amply illustrated in the SUMER atlas (Feldman et al. 2003). Figure 13 displays an exemplary image of the magnetic network as seen in the emission line of C III.

From space missions such as Helios, Ulysses, Yohkoh, SOHO, or TRACE, it has become abundantly clear that three characteristic types of the solar corona and related solar wind exist, and variably prevail at different heliographic latitudes and longitudes over the solar cycle (for an introduction to the corona we refer to Sect. 2.1.4). The solar wind comes in the form of (1) a steady fast flow (from a coronal hole with $T_{\mathrm{e}} \approx 9 \times 10^{5} \mathrm{~K}$ ), (2) a slow wind (from the quiet-Sun corona with $T_{\mathrm{e}} \approx 1.4 \mathrm{MK}$ ), and (3) transient and sometimes fast solar wind (from active regions or small coronal holes). The most spectacular transient events come in the form of CMEs. The global solar magnetic field, as shown in Fig. 12, and the related heliospheric flow pattern are most simple near solar minimum, when fast streams emanate from open polar coronal holes, and slow streams originate from the cusps and boundary layers of the equatorial streamer belt. The closed corona (active loops and prominences; see Sects. 2.2.2.1 and 2.2.2.4) may intermittently open up and abruptly produce huge CMEs (discussed in Sect. 2.2.2.7) and magnetic flux ejections. The coronal origins of the solar wind and the sources of steady streams and transient flows caused by magnetic eruptions were briefly reviewed by Marsch (2006a), and the solar wind response to the activity cycle was reviewed by Marsch (2006b). Based on an International Space Science Institute (ISSI) study, an account of the present status of CME research was given by Schwenn et al. (2006).

The interplanetary wave and turbulence pattern associated with the various types of solar wind flows (reviewed by Tu and Marsch 1995, and others) closely reflects the coronal boundary conditions. Fast streams are permeated by large-amplitude Alfvén waves (with flat spectra, index: -1) accompanied by low-level sonic noise, whereas slow streams often carry sizeable compressive fluctuations (with Kolmogorov spectra, index: $-5 / 3$ ) show hardly any Alfvén waves, but convect embedded pressure-balanced structures, such as magnetic flux tubes and sharp discontinuities. In contrast, CMEs are usually characterized by strong and quiet magnetic fields, with neither magnetosonic nor Alfvénic fluctuations. The most recent and complete review, including the phenomenon of intermittency, on solar wind turbulence, was written by Bruno and Carbone (2005). 
The solar origins and causes for the apparent differences in the wave and turbulence patterns of the solar wind remain largely unknown. Concerning waves in the corona itself, the basic theory of MHD waves was developed a quarter of a century ago; its verification lacked adequate coronal observations. They are, however, available today. Facing the recent observations, this theory has been revisited and expanded, and reviewed extensively by Roberts (2002, 2003) and Roberts and Nakariakov (2003). For example, based upon the observations coming mainly from the TRACE and SOHO missions, coronal oscillations were in the past few years observed and theoretically analysed in much detail. Thus, a new field of solar physics developed, coronal seismology, to which an introduction was given by Nakariakov (2003).

\subsection{The Magnetic Transition Region-Confinement and Release of Coronal Plasma}

The classical concept of a thermally stratified transition region is not verified by observations. In actuality, this crucial region has an extremely inhomogeneous structure, and has been probed for the first time by the SUMER spectrograph, estimating the height profile of the proton temperature from the neutral hydrogen temperature through the Lyman line series. This magnetic transition region which lies between about $2 \mathrm{Mm}$ and $10 \mathrm{Mm}$ above the photosphere is reviewed.

\subsubsection{The Classical Temperature Transition Region}

The original concept of a transition region of the solar atmosphere was based on the idea of a uniformly stratified (onion-shell) and layered atmosphere, in which the temperature jumped within a few hundred kilometres from a temperature of $1 \times 10^{4} \mathrm{~K}$ typical for the upper chromosphere to $1 \mathrm{MK}$ typical for the corona. This geometry is essentially one-dimensional and does not apply to the magnetically structured solar atmosphere consisting of loops and funnels. The classical transition-region physics was described extensively by Mariska (1992). This traditional concept of the transition region is illustrated in Fig. 1. The thermodynamics of such a static transition layer essentially includes heat conduction (mostly from the hot corona) that balances the radiative losses and adds to a direct heating of that layer. All this implies a strong temperature jump occurring in the model on very small scales (of about $100 \mathrm{~km}$ ). Yet no one has ever verified this observationally. Does that classical transition region exist at all, or does it perhaps only occur locally along a single magnetic field line?

SUMER has for the first time obtained a height profile in this crucial region of the proton temperature, which was inferred from the neutral hydrogen temperature that was derived from the Lyman line series (Marsch et al. 2000). The theoretically predicted sharp temperature jump was not found. The measurements, covering an altitude range from $12 \mathrm{Mm}$ to $18 \mathrm{Mm}$, apparently represented a mixed data sample obtained by integration over an array of cold and hotter adjacent loops (with a broad range of loop sizes) that were visible along the LOS.

Marsch et al. (2006) recently investigated the magnetic structure of the solar transition region as observed in various ultraviolet lines emitted at different formation temperatures in a coronal hole. Their emission height analysis confirms the notion that plasma can, at different temperature, coexist at the same height. The transition region is not simply thermally stratified but strongly nonuniform. How can one reconcile all these findings with a conventional transition region? This question remains to be answered by future studies. 


\subsubsection{The Complex Magnetic Transition Region}

The real structure of what we may call the magnetic transition region is suggested observationally by the overall emission pattern in various VUV lines. Therefore, we may define the magnetic transition region to be that layer of the solar atmosphere which lies between about $2 \mathrm{Mm}$ and $10 \mathrm{Mm}$ above the photosphere, where the temperature increases along each field line, and the magnetic field itself strongly changes its topology and thereby becomes more uniform. Only structures on scales of $10 \mathrm{Mm}$ and larger will finally remain and form the global coronal magnetic field. The images obtained by VUV imagers and spectrometers apparently indicate that the magnetic transition region is made up of an entangled array of closed and open fields. The complex global transition of the solar magnetic field with height was shown in Fig. 12. It is again illustrated, in a cartoon fashion and on a smaller scale, in the two panels of Fig. 14, which shows the carpet of loops intermingled by funnels, and illustrates the active magnetic network with reconnection.

All these magnetic structures, of which only the network is schematically shown here, together constitute a complex field, in which turbulence and waves may be excited, e.g., by reconnection or footpoint motion of field lines tied to the photosphere. While the waves may escape to the heliosphere on open field lines, they will remain trapped in the corona when bouncing in closed loops.

Another detailed evaluation of the active transition-region structure comes from TRACE observations (De Pontieu et al. 2003). What has been named moss (discussed in Sect. 2.2.2.4) and relates to bright emissions above plages, may provide a powerful tool to probe the magnetic coupling between the chromosphere and the transition region. A cartoon of the conceptual view of the magnetic transition region is given in Fig. 15, which summarizes various dynamic features of the magnetic transition region as compared to the classical temperature transition region. The field lines constituting a coronal loop may be anchored in many disparate magnetic elements in the magnetic transition region.

However, there remain many open questions with respect to the magnetic transition region and its temperature distribution, such as:

(1) How do the small-scale fields and flux tubes (with a typical scale of $100 \mathrm{~km}$ ) evolve with height within the first few megametres?

(2) How does the transition to a more uniform (on the scale of supergranules) coronal magnetic field actually take place? Can this transition somehow be traced (such as for the moss emission) by the morphology of the VUV emission in lines of different temperatures?

(3) How can the observational results as summarized in the SUMER atlas be consistently interpreted, in particular concerning the apparent small loops bridging the network?

(4) How is the flow of plasma into the corona through the magnetic transition region organized and guided by the field? The replenishment of coronal plasma on open field lines against solar wind losses, and on closed field lines against gravity, which would force loops and streamers to be emptied by coronal rain, requires upward flows everywhere and continuously.

(5) Is there some systematic large-scale flow pattern in the magnetic transition region? If yes, how is this correlated with the temperature increase with height?

Coronal plasma flows and magnetic fields in active regions were analysed by Spadaro et al. (2000) and Marsch et al. (2004), combining magnetograms with SUMER observations. It was found, by means of force-free magnetic field extrapolation, that the radiance and Doppler-shift patterns of various transition-region lines are closely correlated with the 


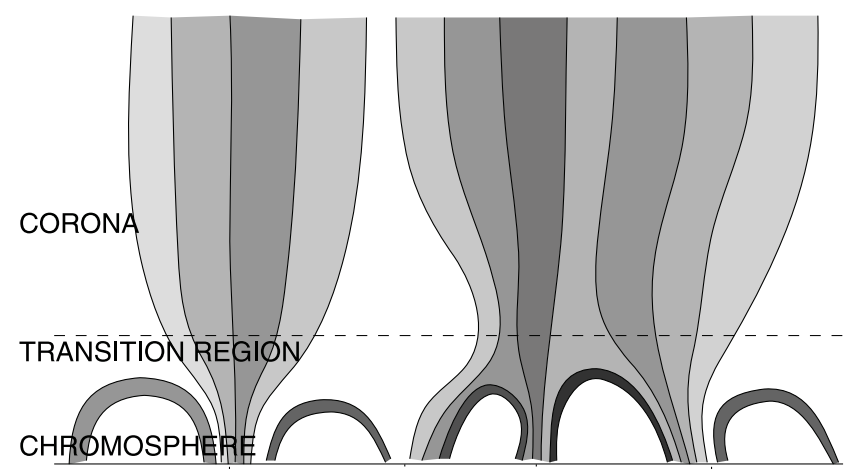

PHOTOSPHERE
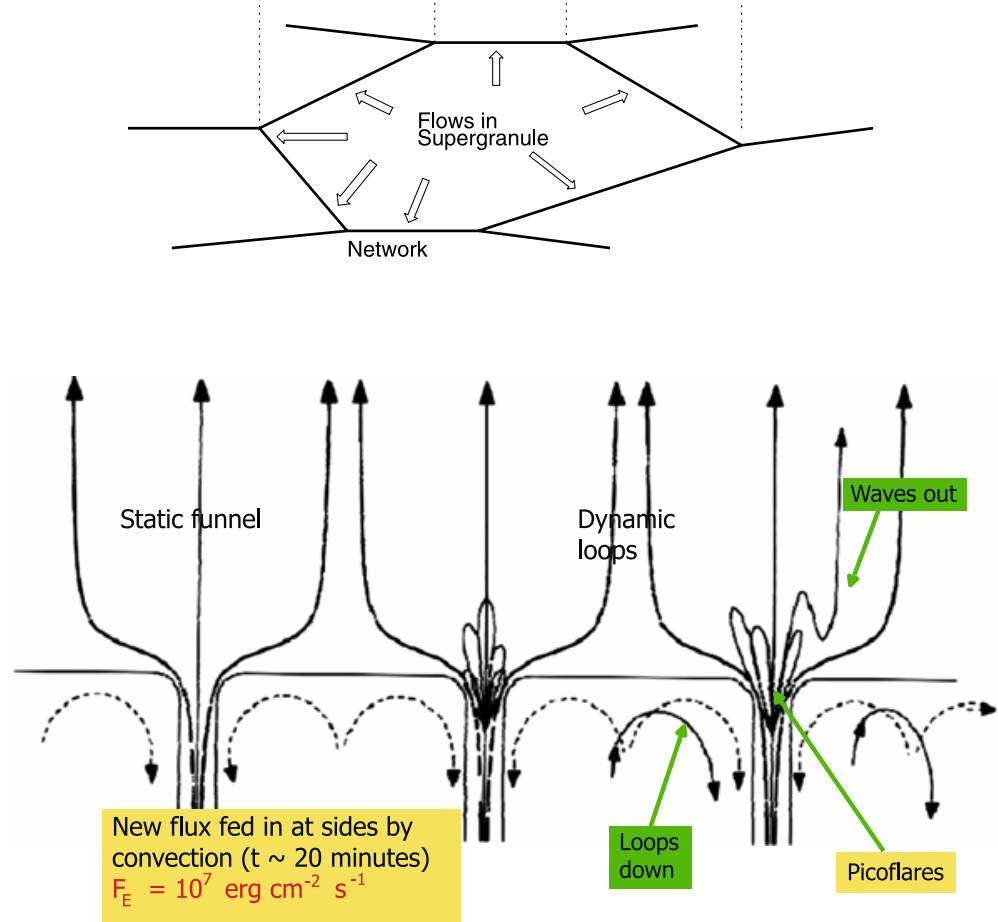

Fig. 14 Top: Schematic presentation (after Aschwanden 2004) of the building blocks of the magnetic network with its main structures, closed loops and open funnels. The fields originating in the network lanes may be open or close, thus covering the supergranulation cells or providing magnetic flux to the upper corona. Bottom: The dynamic network as a wave and turbulence generator through convection-driven reconnection (after Axford and McKenzie 1997)

magnetic field topology and strength. They identified up- and down-flows in sunspots and regions with small magnetic gradients and strong velocity shears, and also derived estimates of the coronal current density. Similar studies in coronal holes showed the sources of the fast solar wind and the height of its initial outflow between $5 \mathrm{Mm}$ and $20 \mathrm{Mm}$ (Tu et al. 2005a), a result discussed in detail later. All these studies elucidated the primary rôle the field plays in constraining and guiding coronal plasma flows. 


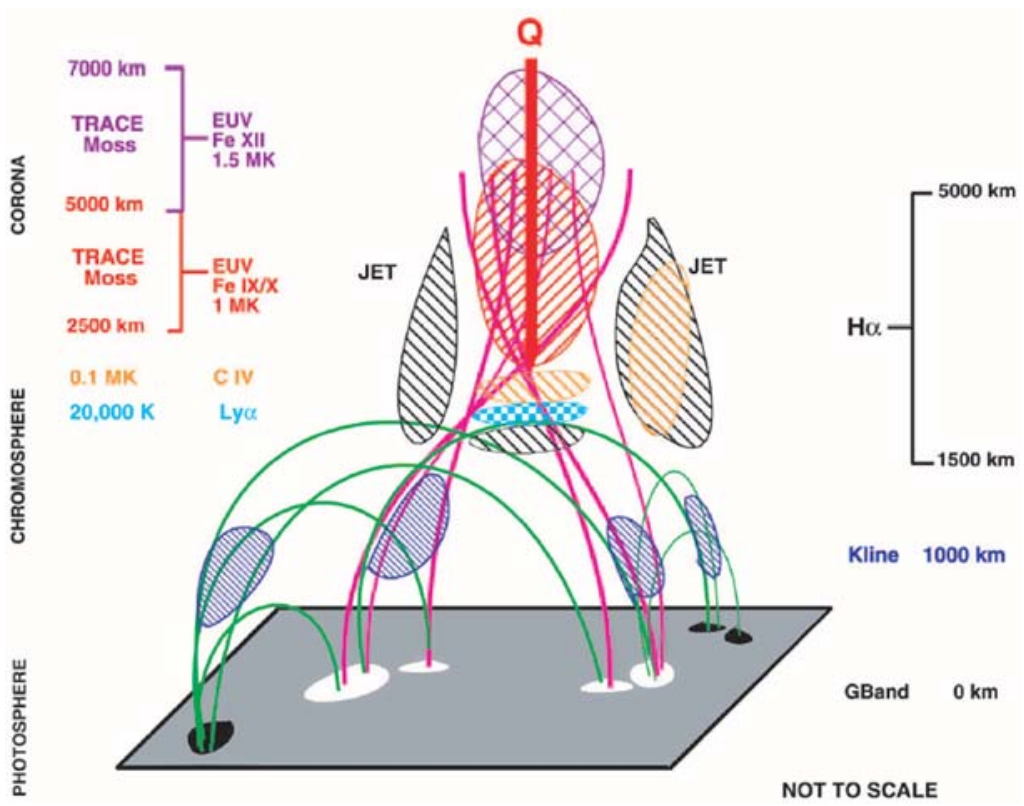

Fig. 15 Schematic cartoon of the solar transition region (after De Pontieu et al. 2003) with various emission regions being indicated and magnetic structures outlined. Several ultraviolet lines associated with moss emission are also indicated, and their formation temperatures and estimated heights of origin are given on the margins

\subsection{Waves and Flows in Loops and Funnels}

Understanding of the energization processes in the chromosphere and transition region holds the key to solving the problem of coronal heating and solar wind acceleration. This section first reviews the novel correlative analyses by combining solar magnetograms and Dopplergrams. The magnetic network is presented as a site for not only wave generation and turbulence, but also a prolific source of the solar wind energy, mass and magnetic flux that fills the entire heliosphere. We then review how the measurements of Doppler shifts and widths of VUV lines have renewed the interest in waves and oscillations in the solar atmosphere, and thereby understanding the physics of the Sun's atmosphere.

\subsubsection{Magnetograms and Dopplergrams}

Novel correlative analyses were carried out by combining solar magnetograms with images from TRACE and Dopplergrams from SUMER. Generally, solar magnetograms clearly indicate that the magnetic network field exists in two main characteristic components, i.e., in unipolar flux tubes (funnels) and in closed tubes (loops) (see Fig. 14). The small loops (at a scale of $1 \mathrm{Mm}$ and smaller) will emerge or submerge and collide with each other, and thus will constitute a permanent source of energy for the corona. The small-scale activity of this magnetic carpet, and the dynamics of ephemeral regions and their consequences for coronal heating were discussed by Schrijver et al. (1997, 1998) and Aschwanden (2001), who concluded that the coronal heating problem might only be solved with the help, and through the understanding, of the energization processes in the chromosphere and lower transition region. 
Fig. 16 Photospheric magnetogram and outflow of neon ions inferred from SUMER observations of Doppler blue shifts of Ne VIII $(77 \mathrm{~nm})$ relative to $\mathrm{C}$ I $(154.2 \mathrm{~nm})$ in an equatorial coronal hole. Blue contours: dotted $10 \mathrm{~km} \mathrm{~s}^{-1}$, solid, $7 \mathrm{~km} \mathrm{~s}^{-1}$; white contours: solid $3 \mathrm{~km} \mathrm{~s}^{-1}$, dotted, $0 \mathrm{~km} \mathrm{~s}^{-1}$. KPNSO magnetogram: white, positive, black, negative field polarity. The grey scale (top bar) ranges from $-100 \mathrm{G}$ to $+100 \mathrm{G}$. Note the close correlation between blue shifts and positive fields, indicating significant upflows in open unipolar magnetic regions (after Xia et al. 2003)

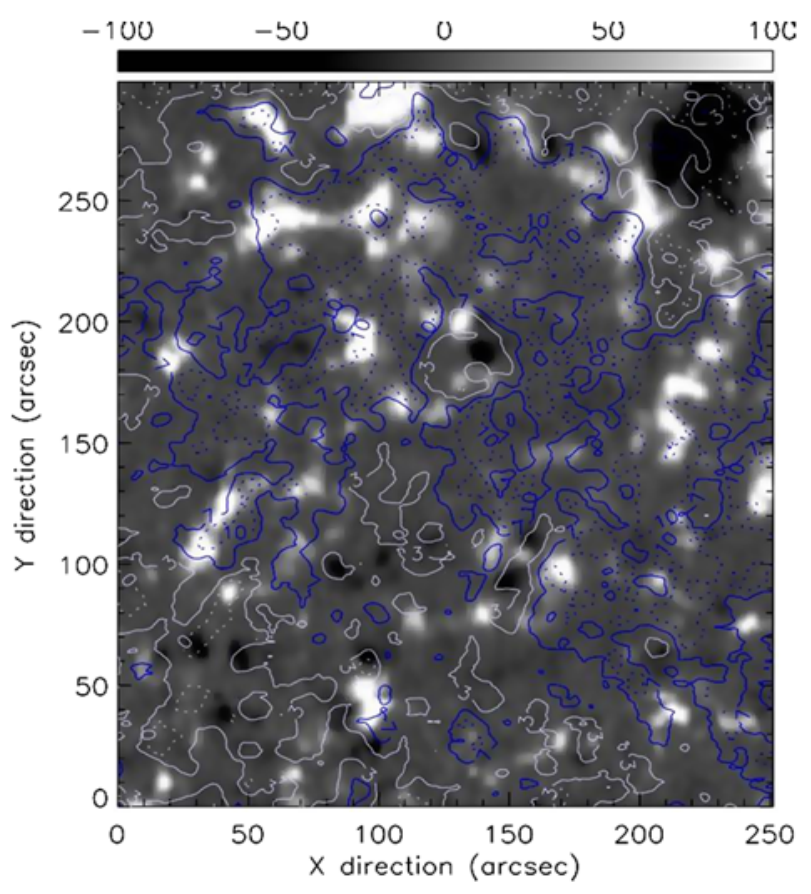

The magnetic network is the key building block of the magnetic transition region. It is not only a site of continuous generation of waves and turbulence by reconnection as well as shaking of flux tubes anchored in the network, but it is also the prolific source of the solar wind energy, mass and magnetic flux that fills the whole heliosphere. Indications for the outflow of the nascent solar wind in association with the photospheric magnetic field are presented in Fig. 16, which shows a magnetogram of an equatorial coronal hole analysed by Xia et al. (2003). The overlaid contours of the sizeable Doppler shift of neon ions clearly indicate blue shifts, i.e., outflows, at the cell boundaries and lane junctions in the network below the coronal hole.

The detailed source regions of the fast solar wind were recently identified by Tu et al. (2005a) as the funnels in a coronal hole, i.e., the expanding magnetic field structures rooted in the magnetic network lanes. These authors corroborated and complemented earlier work by Hassler et al. (1999), and established that the solar wind starts flowing out of the corona at heights above the photosphere between $5 \mathrm{Mm}$ and $20 \mathrm{Mm}$ in the funnels. This result was obtained by a correlation of the Doppler-velocity and radiance maps of different spectral lines. Specifically, $\mathrm{Ne}^{7+}$ ions were found to radiate mostly around $20 \mathrm{Mm}$, where they have outflow speeds of about $10 \mathrm{~km} \mathrm{~s}^{-1}$, whereas $\mathrm{C}^{3+}$ ions with no average flow speed radiate mainly around $5 \mathrm{Mm}$. These findings are illustrated in Fig. 17, which shows in two planes colour-coded maps of the magnetic field strength at a height of $4 \mathrm{Mm}$ and $20 \mathrm{Mm}$. Regions with outflow speed larger than $7 \mathrm{~km} \mathrm{~s}^{-1}$ are indicated by the hatched patches that coincide with the open unipolar field of the funnel.

Stimulated by these observations, Tu et al. (2005a) suggested a new model to explain the origin of the fast solar wind. The transition region in coronal holes is full of magnetic loops of different sizes, mostly with a height of less than $5 \mathrm{Mm}$. The supergranular convection in the photosphere keeps the feet of these loops moving and thus transfers kinetic energy to magnetic energy that is stored in the loops. The loops may finally collide with the funnel 


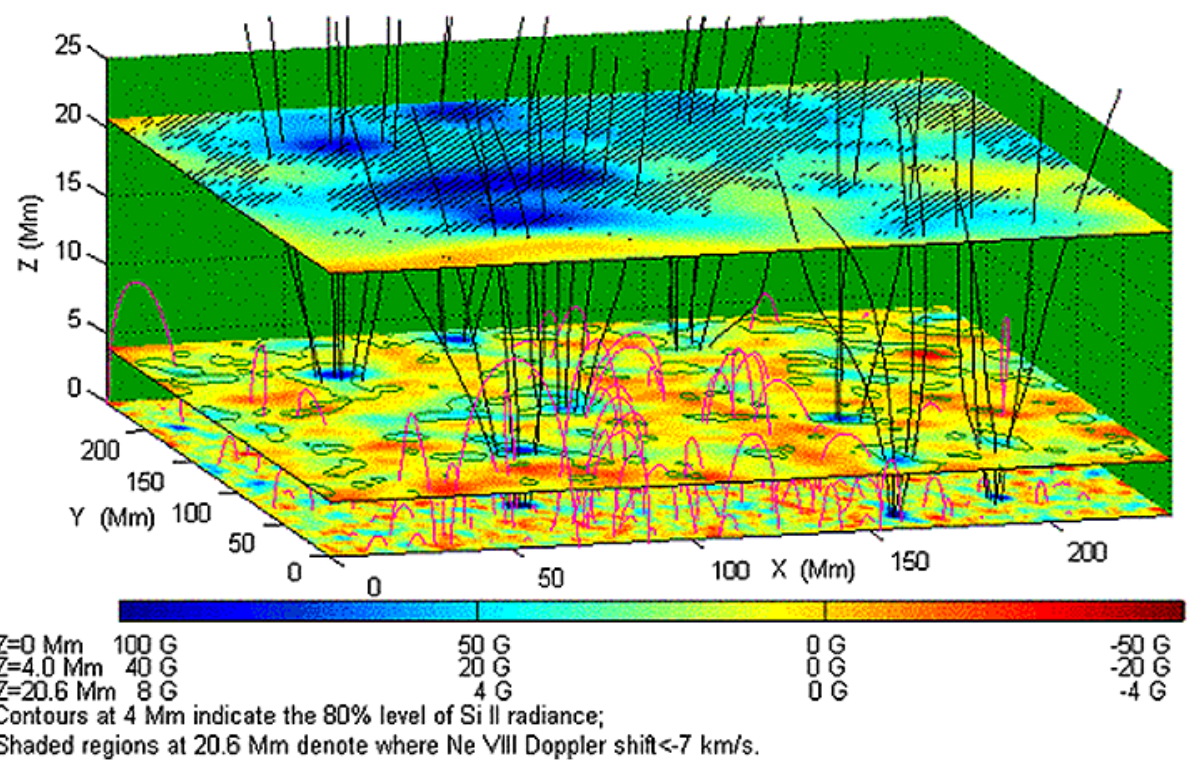

Fig. 17 Source regions of the fast solar wind in the magnetic funnels of a coronal hole. The magnetic field magnitude is shown in two planes at $4 \mathrm{Mm}$ and $20 \mathrm{Mm}$. The hatched areas indicate outflow speeds of neon ions larger than $7 \mathrm{~km} \mathrm{~s}^{-1}$. The open field is indicated by black lines, and the closed loops in magenta colour. They hardly reach a height of $10 \mathrm{Mm}$ (after Tu et al. 2005a)

and reconnect there with pre-existing open field. Thereby, the plasma is released from the loops, a process that may lead to both upflows and downflows. Ultimately, parts of the plasma contained in reconnecting loops are brought into the corona. In the lower transition region below about $5 \mathrm{Mm}$, we mainly have horizontal exchange of mass and energy between neighbouring flux tubes, which is driven by supergranular motion. Above $5 \mathrm{Mm}$ or higher, where reconnection between field lines of funnels and surrounding loops gradually ceases, vertical transport will become more important than horizontal, and the radial acceleration of the solar wind will actually start.

This scenario, illustrated in Fig. 17, is a further development of the magnetic furnace idea of Axford and McKenzie (1997). Numerical simulations also indicated that magnetic reconnection between slowly rising loops and funnels can in fact take place and may be responsible for the delivery of mass and energy to a funnel. That reconnection in the dynamic magnetic network plays a key rôle in the solar wind plasma supply was confirmed by several direct numerical simulations and model calculations made by Büchner and collaborators (Büchner et al. 2004; Büchner and Nikutowski 2005). These authors developed a physically consistent model for the magnetic transition region and demonstrated that coronal heating is possible as the result of reconnection (occurring at about $5 \mathrm{Mm}$ ), which was rendered possible by anomalous (via current-driven micro-instabilities) resistivity. As a further result, plasma was accelerated upwards (Büchner et al. 2005) to speeds of about $10 \mathrm{~km} \mathrm{~s}^{-1}$, a value that appears consistent with the Doppler-shift observations.

\subsubsection{Doppler Shifts and Widths}

It is difficult to observe coronal plasma waves directly, because the plasma diagnostics available for the solar atmosphere is still insufficient. In-situ measurements, such as those made 
in other solar-system plasmas (e.g., in the solar wind), are impossible in the corona. Yet, as shown in previous sections, remote sensing of atmospheric phenomena is feasible. The waves manifest themselves in spectral lines through radiance (i.e., electron density) modulations and the Doppler effect, which may lead to line shifts that are either resolved as flows or unresolved as thermal and turbulent broadenings. For a detailed discussion of the observations we refer in particular to Sect. 2.1.3. For a comprehensive review of VUV spectroscopy and coronal diagnostics, see Part I of this review. Here we closely follow in part the previous article by Marsch (2004) on waves and turbulence in the solar corona.

Concerning the largest resolvable wave scales, it is required that the wavelength $\lambda \ll L$ and period $P \ll D$, where $L$ is the extent of the field of view and $D$ the duration of an observational sequence. This requirement is readily fulfilled by modern instruments. Also, their spectral resolutions are sufficient to measure Doppler shifts and broadenings (see Part I and Sect. 2.1.3.1). The integrated effects of the resolved low-frequency waves or the unresolved high-frequency turbulence are observationally considered in the LOS turbulent amplitude $\xi$.

Ample evidence was in the recent past provided by TRACE and SOHO for the existence of waves trapped in loops or propagating in flux tubes (see, e.g., Aschwanden et al. 2002; Wang 2004) and plasma flowing out in open funnels to the outer corona. For this outflow see again the references in Xia et al. (2003), and inspect the results shown in Fig. 16 and Fig. 17. Coronal funnels and their associated ion flows were modelled by Marsch and Tu (1997b), Hackenberg et al. (1999, 2000). In their two-fluid model including waves they considered ion heating, which was assumed to be caused by cyclotron-wave sweeping. Invariably, a steep inward temperature gradient resulting from electron heat conduction was also found, as it is typical for the classical transition region that is illustrated in Fig. 1.

Forward modelling of funnels was recently performed by Aiouaz et al. (2005b) with the intention of predicting the observed VUV emission. Aiouaz and Rast (2006) further studied the effects of a mixed-polarity background field on the network expansion with height through the solar corona and found that it deviated significantly from the simple funnel expansion model. Similarly, Esser et al. (2005) calculated the Lyman- $\alpha$ emission from a dynamic coronal funnel, and obtained that the emission was enhanced, due to the enthalpy flux in the funnel, by a factor of up to 10 as compared to a static stratification.

Theoretically, coronal waves confined in loops are-when being excited, for example, by flaring at the loop apex-expected to travel from loop top to bottom and thus will produce mainly red shifts. On the contrary, the plasma streaming out on open field lines is supposed to come from the chromosphere, and thus produce mainly blue shifts. Concerning the observational evidence, both expected types of Doppler shift have indeed been observed (Seely et al. 1997; Marsch et al. 1997; Tu et al. 1998; Peter and Judge 1999; Tu et al. 1999; Hassler et al. 1999; Wilhelm et al. 2000; Peter 2001; Xia et al. 2003, and references therein).

Surveys of the average observational situation are given in Figs. 5, 9 and 18, the latter showing the line widths separately for the core and wing parts, versus the line-formation temperature, which is essentially the electron temperature in the range from $4 \times 10^{4} \mathrm{~K}$ to $6.3 \times 10^{5} \mathrm{~K}(\lg T / \mathrm{K}=4.6$ to 5.8$)$ values corresponding to heights below $1.3 R_{\odot}$. Note the distinct change from red to blue shifts in Fig. 9 with increasing temperature to the latest studies.

Considering the Doppler widths as presented in Fig. 18 taken from Peter (2001), we note a clear separation in line width between the core and tail components for all the lines shown, with the width typically ranging between $20 \mathrm{~km} \mathrm{~s}^{-1}$ and $30 \mathrm{~km} \mathrm{~s}^{-1}$ in the core (lower points) but steadily growing with height in the corona from about $40 \mathrm{~km} \mathrm{~s}^{-1}$ to $90 \mathrm{~km} \mathrm{~s}^{-1}$ in the line tail (upper points). The major contributions, to perhaps the core but certainly the tail component, of the line broadening come from $\xi$, which is an empirical wave amplitude that 
Fig. 18 Emission-line widths corresponding to wave amplitudes as a function of height in the lower corona. The widths of the core component (shown in blue colour) and tail component (shown in red colour) of each line are plotted separately. The Alfvén speed $\left(0.2 V_{A}\right.$ : dashed line $)$ and sound speed (continuous line) are given as well (after Peter 2001)

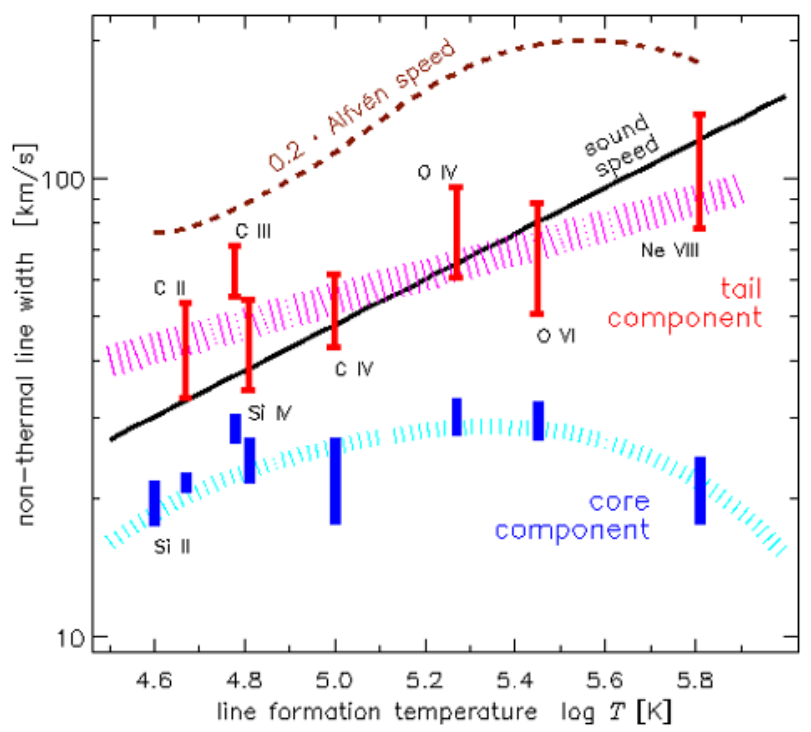

is conveniently used to estimate the amount of turbulent wave energy existing in the corona (cf., Sect. 3.5, Eq. 35 of Part I). Marsch et al. (1997) and Wilhelm et al. (1998b) have also analysed the radial profile of $\xi$ off the solar limb in a coronal hole and found an increase of $\xi$ going up to values of $40 \mathrm{~km} \mathrm{~s}^{-1}$ to $50 \mathrm{~km} \mathrm{~s}^{-1}$ as inferred from $\mathrm{Mg} \mathrm{X}(62.5 \mathrm{~nm})$ at $1.1 R_{\odot}$, and $80 \mathrm{~km} \mathrm{~s}^{-1}$ from Si VIII $(144 \mathrm{~nm})$ at about $1.3 R_{\odot}$.

\subsection{Heating of the Solar Coronal Holes}

SOHO's VUV data provide several clues to the heating mechanisms of the corona and coronal holes. In terms of proton and heavy ion kinetic temperatures, coronal holes are the hottest places on the Sun. The solar wind is found to emanate from the chromospheric network. The pervasive steady heating of the corona in active and quiet regions as well as in coronal holes may be caused by explosions of sheared magnetic fields in the cores of initially closed bipolar loops. The nonthermal VUV line broadenings are interpreted as signatures of ion heating caused by cyclotron wave absorption, though realizing that both theoretical work and further observations are needed to explain proton and heavy-ion heating of the corona. This section reviews the different heating mechanisms, such as heating by network flaring and cyclotron-wave heating of coronal ions.

\subsubsection{Heating by Network Flaring}

The corona apparently is hot even when there is no obvious magnetic activity. This general notion may indicate uniformity of the heating process. SOHO has shown that coronal holes (see also Sect. 2.1.4.2) are, in terms of their proton and heavy ion kinetic temperatures, the hottest places on the Sun (Kohl et al. 1997; Wilhelm et al. 1998b; Tu et al. 1998). Observationally, the magnetic (chromospheric) network looks the same in cool lines beneath the closed (quiet) corona and open holes, but it differs substantially when seen in hot transitionregion lines (Hassler et al. 1999). The energy flux required to power the fast solar wind and the energy flux needed to produce the quiet magnetically confined corona (mainly compensation for radiative losses) are both about $5 \times 10^{2} \mathrm{~J} \mathrm{~m}^{-2} \mathrm{~s}^{-1}$. It therefore seems natural to postulate the energy source to be about the same all over the quiet Sun. 
In the Parker model of quiet coronal heating by "nano-flares", low-frequency waves are generated from the twisting and footpoint shuffling of magnetic field lines, whereby the stored energy is intermittently released by magnetic reconnections in the corona (Parker $1958,1983,1988)$. This is equivalent to a flux of very low-frequency waves, which are hard to dissipate and cannot, according to an extrapolation back to the Sun of their energy flux measured in the fast solar wind, provide the necessary energy flux at the coronal base (Tu and Marsch 1995). Therefore, these waves do not seem to be an adequate source for coronal heating.

SOHO observations in the polar coronal holes indicate that the solar wind emanates directly from the chromospheric magnetic network (Hassler et al. 1999; Wilhelm et al. 2000; Xia et al. 2003; Tu et al. 2005a), with relatively high upward initial speeds. Below the coronal base, the magnetic field (of about $1 \mathrm{mT}$ ) is anchored mainly in the supergranular network, which occupies merely $10 \%$ of the base area in holes. In the network lanes the magnetic pressure generally dominates the thermal pressure. The network magnetic field (of about $1 \mathrm{mT}$ to $10 \mathrm{mT}$ ) is rooted in the photosphere in small, flux tubes (with fields of about $100 \mathrm{mT}$ and $100 \mathrm{~km}$ in size), expands rapidly with height in the transition region and then fills the entire overlying corona (see Fig. 14).

The magnetic network field exists in two major side-by-side components, i.e., in uncancelled unipolar flux tubes (funnels) and in myriads of bipolar loops. The only conceivable energy source for the solar corona is associated with the dynamics of the network field itself, as an open-funnel potential field (Gabriel 1976) has no free energy, and a closed-loop static field (Dowdy et al. 1986) also does not release magnetic energy. Yet time-varying fields undergoing reconnection (Axford and McKenzie 1997) will cause flaring and provide energy to the corona. The loops will emerge or submerge and frequently collide, and thus constitute a permanent source of magnetic energy. This scenario is illustrated in the lower panel of Fig. 14.

Moore et al. (1999) built a complex case for pervasive steady heating of the corona in active and quiet regions, and in coronal holes, all being driven in basically the same way as during the intense transient heating in solar active regions and flares, namely by explosions of sheared magnetic fields in the cores of initially closed bipolar loops. Their scenario is illustrated in Fig. 19. They invoked myriads of micro-explosions that drive in situ coronal heating via reconnection. The internal convulsion and reconnection of the magnetic field in these explosions may also generate MHD waves that heat coronal holes around the core fields, and in addition may inject energetic particles and hot plasma into extended loops.

We assume that these "microflares", and even smaller "picoflares", are all self-similar to other intra-network flares in the sense that remnant closed loops on all scales containing hot VUV emitting plasma are produced. Network flares must occur frequently, and perhaps have a dimension down to the presently unresolved $10 \mathrm{~km}$ to $100 \mathrm{~km}$ scale. Concerning their overall energetics and possible rôle in the generation of the solar wind, see the reviews by Axford et al. (1999) and Marsch et al. (2003). The energy burned in the "magnetic furnace" must be replaced by magneto-convection in the photosphere, which ensures adequate supply of randomly looped magnetic flux. If the energy thus generated by magnetic reconnection is released with a typical value of about $10^{14} \mathrm{~J}$, then such small-scale magnetic activity is also expected to continually produce a sizable amount of waves at relatively high frequencies (larger than $1 \mathrm{~Hz}$ ). Wave dissipation would rapidly occur within a fraction of a solar radius and could involve an essentially linear mechanism, such as cyclotron damping. 


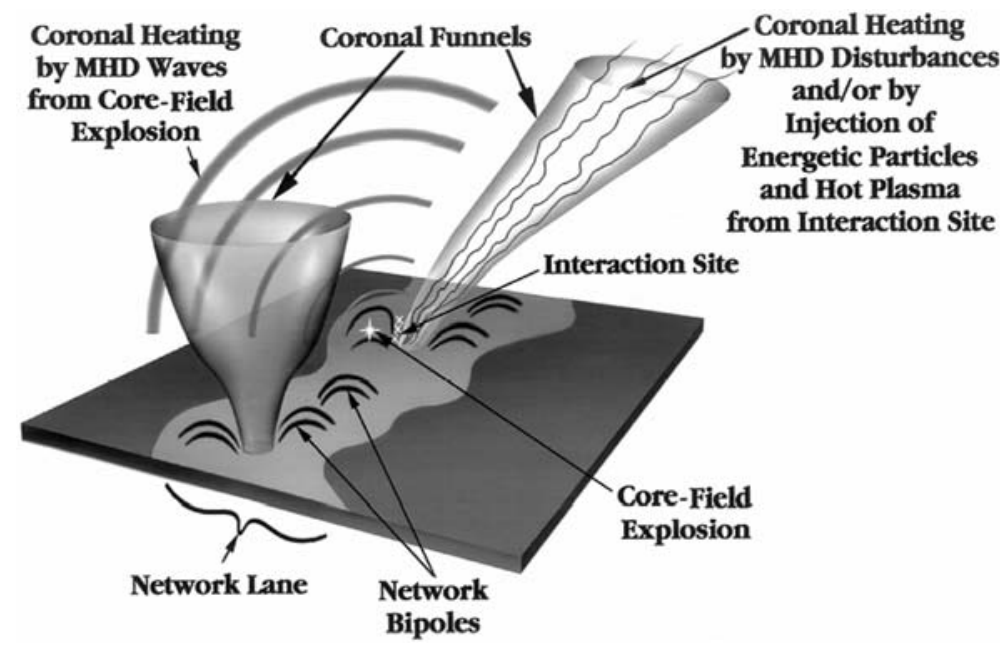

Fig. 19 The scenario for heating the corona in quiet regions and coronal holes by core-field explosions in network bipoles after Moore et al. (1999). The bipoles are concentrated in and around the network magnetic flux concentrations, and so many are embedded in or impacted against the feet of coronal magnetic funnels, giving a combined field configuration like that of a flaring arch or coronal X-ray jet: an active bipole impacted in one end of a much larger passive magnetic arch or funnel

\subsubsection{Cyclotron-Wave Heating of Coronal Ions}

The idea that cyclotron waves might heat the ions in coronal holes has been worked out in the recent past by several authors. Their work was reviewed by Hollweg and Isenberg (2002), to whom we must refer here for an in-depth study of this subject. They also considered the generation of the fast solar wind, but with emphasis on the cyclotron-resonant acceleration of the protons and heavy ions. The empirical model of Cranmer et al. (1999b) places spectroscopic constraints from SOHO on the cyclotron heating mechanism of coronal holes. This idea was corroborated in a two-fluid wave-driven solar wind model by Tu and Marsch (1997), including parametric studies of the resulting wind properties in dependence on the average wave amplitude at the coronal base (Marsch and Tu 1997a). A key feature of this model is that the damping of Alfvén waves at the cyclotron frequency in a rapidly declining magnetic field (frequency sweeping), can provide strong heating close to the Sun. In all the models, assumptions had to be made about the unknown spectrum of the waves injected at the coronal base. A power-law spectrum constrained by extrapolation of the in-situ measurements (see, e.g., Tu and Marsch 1995) has usually been assumed.

High-frequency waves of coronal origin, which can resonantly heat the ions, and in particular preferentially accelerate minor ions with respect to the protons, have been invoked to explain the measured characteristics of ion velocity distribution functions (VDF) in the solar wind and coronal holes, and to interpret spectroscopic evidence obtained from VUV emission line measurements made by SOHO, since they indicate considerable line broadenings (Kohl et al. 1997; Tu et al. 1998) and sizable Doppler shifts. The preferential heating and acceleration of $\mathrm{O}^{5+}$ ions, as observed in coronal holes by UVCS (Kohl et al. 1998), have been explained by wave-particle cyclotron resonance. Cranmer (2000) in particular investigated the ion-cyclotron wave dissipation in the solar corona by consideration of the summed absorption of more than 2000 ionic species with fixed Maxwellian VDFs. 
An alternative explanation of the observed line width of oxygen was suggested by Raouafi and Solanki (2003, 2004), who studied the effect of anisotropic velocity distribution on the linear polarization of coronal lines. Based on their results from global modelling of the coronal magnetic field, they concluded that the observed line broadening could have been caused by unresolved field-aligned bulk flow, and they therefore critically questioned whether the ion-cyclotron heating really existed in the inner corona.

The quasi-linear theory (QLT) (Kennel and Engelmann 1966) of wave-particle interactions, which involve diffusion of the particles by pitch-angle scattering in the broad-band and random-phase wave fields, allows the effects of resonant interactions between ions and waves to be calculated. By taking moments of the quasi-linear diffusion operator, the parallel and perpendicular wave heating and acceleration rates for gyrotropic particle VDFs were derived in several papers (Marsch and Tu 2001a, 2001b; Tu and Marsch 2001a, 2001b). These rates can be used in anisotropic multi-component fluid equations to describe the interactions of ions with, for example, kinetic Alfvén or ion-cyclotron waves. Tu and Marsch (2001a) showed that a self-consistent treatment of the wave damping and absorption is absolutely necessary.

A semikinetic model was developed (Vocks and Marsch 2001, 2002) for the dynamics of ions in the solar corona. This model consists of a closed set of diffusion equations. It includes wave-particle interactions within the framework of quasi-linear theory and Coulomb collisions calculated by using the Landau collision integral. The integration of the VDFs over the velocity components perpendicular to the background magnetic field yields so-called "reduced" VDFs. Coupled Vlasov-Boltzmann equations for these reduced VDFs were derived which could be solved numerically with reasonable effort.

The SOHO observations of nonthermal VUV-line broadenings have been interpreted as signatures of ion heating caused by cyclotron-wave absorption. The multifluid and semikinetic modeling of these phenomena lead to the convincing conclusion that, given sufficient wave power exists at the coronal base (i.e., at the funnel base in a coronal hole), this process could indeed yield significant ion heating. To obtain a conclusive result, a consistent calculation of the wave opacity (fully based on kinetic theory) is required. However, the observations discussed in previous sections have not been satisfactorily explained as long as the direct evidence for the invoked coronal plasma waves is still lacking. Certainly, both theoretical work and further observations are needed to explain proton and heavy-ion heating in the corona.

\subsection{Acceleration of the Solar Wind}

The solar wind is the supersonic outflow of completely ionized gas from the solar corona. It consists of protons and electrons with a relative admixture of a few percent of $\alpha$ particles and heavy, much-less-abundant, ions in different ionization stages. In the open field regions, the hot corona is at typical electron temperatures of $1 \mathrm{MK}$ to $2 \mathrm{MK}$ and expands into interplanetary space. The flow becomes supersonic at a few solar radii. For a recent review of solar wind theory see Marsch et al. (2003). In this section, we closely follow that paper and also exploit the previous article by Marsch (1999) on solar wind models from the Sun to 1 ua and on the constraints imposed by in-situ and remote-sensing measurements.

From various space missions it has become abundantly clear that two characteristic types of solar corona and wind (for the details we refer to the previous Sect. 2.1.4) exist, prevailing at different heliographic latitudes and longitudes and phases of the solar cycle (for concise reviews see, e.g., Marsch 2006a, 2006b). The field and flow pattern is simplest near solar minimum, when the steady fast wind emanates from the polar coronal holes, whereas the 
unsteady slow wind originates from the equatorial streamer belt [which may intermittently open (Wang et al. 1998) to release abruptly CMEs and smaller plasmoids] or perhaps more continuously from the boundary layers of the streamer belt forming the heliospheric current sheet. The expansion of the coronal magnetic flux tubes, extending from high to low heliographic latitudes (Wang and Sheeley 1990), thereby plays a decisive rôle.

\subsubsection{Basic Model for the Solar Wind}

The minimum effort in modelling the fast solar-wind flow is to consider a combined electron-proton fluid with separate energy equations. In addition, $\alpha$ particles are major players (see, e.g., Hansteen et al. 1997), but heavier minor ions may be treated as test particles. As the observations indicate, the thermodynamics and energetics of the corona and wind are still poorly understood. Increasing the sophistication of solar-wind models, one may include the minor species and anisotropies in the ion temperatures as well as proper-frame differential energy flows, which can be related to skewed VDFs or associated with nonclassical heat conduction in the weakly collisional plasma of the corona.

For the sake of simplicity, we consider a steady coronal expansion and wind with mass density $\rho$, flow speed $V$, pressures $p_{\mathrm{e}, \mathrm{p}}$, and radial magnetic-field component $B_{\mathrm{r}}$. In order to account for the nonradial expansion in coronal holes, a nonradial area function of the flow/flux tube, $A(r)=f(r) r^{2}$, is introduced, where $f(r)$ describes the nonspherical expansion. Realistic estimates of this function were provided by Wang and Sheeley (1990) and by Banaszkiewicz et al. (1998). Empirical values of $f(r)$ may typically range between 2 and 10 (see, e.g., Xia et al. 2003). However, the basic model considered here is only one dimensional, like almost all traditional solar wind models.

The complexities of the ionization and recombination processes in the chromosphere (Hansteen et al. 1997), where the plasma is produced in the first place, and where the mass flux may be determined (Peter and Marsch 1997) are not addressed here. For the evolution of the radial magnetic field component Gauss's law is used, implying that the magnetic flux, $F_{\mathrm{B}}=A(r) B_{\mathrm{r}}$, is constant. One should keep in mind, however, the discussion in Sect. 3.2, where the possible need for fully three-dimensional models of the nascent solar wind near its solar source was discussed.

As we also discussed previously, the network field concentrations are surrounded by a mixed-polarity inter-network magnetic field on the scale of granulation. Aiouaz and Rast (2006) found that the expansion of the network boundary with height may deviate significantly from the simple funnel expansion model. In that case the area function $A(r)$ used in 1-D wind models may have to be reconsidered. Concerning the large-scale global solar wind flow pattern, a new generation of solar wind models has emerged in the past decade. They will not be discussed here, but for reference we just cite the detailed article of Riley et al. (2001), who developed an empirically driven global MHD model of the solar corona and inner heliosphere.

As shown in the following (or see the review of Tu and Marsch 1995), the fast streams are permeated by low-frequency Alfvén waves. These waves exert a wave-pressure-gradient force and can thus accelerate the flow (Belcher 1971; Alazraki and Couturier 1971; Hollweg 1973; Jacques 1978). Also direct acceleration of the ions by cyclotron-resonant absorption of high-frequency waves (Dusenbery and Hollweg 1981; Marsch et al. 1982; Isenberg and Hollweg 1983) may occur. The stationary momentum equation including the Alfvén wave pressure, $p_{\mathrm{w}}$, and cyclotron-wave acceleration, $a_{\mathrm{w}}$, is

$$
V \frac{\mathrm{d}}{\mathrm{d} r} V=-\frac{1}{\rho} \frac{\mathrm{d}}{\mathrm{d} r}\left(p+p_{\mathrm{w}}\right)+a_{\mathrm{w}}-\frac{G M_{\odot}}{r^{2}} .
$$


The thermal particle pressures are, $p_{\mathrm{e}}=n k_{\mathrm{B}} T_{\mathrm{e}}$ and $p_{\mathrm{p}}=n k_{\mathrm{B}} T_{\mathrm{p}}$, and the total pressure is $p=$ $p_{\mathrm{p}}+p_{\mathrm{e}}$. Quasi-neutrality means that $n_{\mathrm{e}}=n_{\mathrm{p}}=n$, and the mass density is $\rho=n\left(m_{\mathrm{p}}+m_{\mathrm{e}}\right)$. The wave pressure is

$$
p_{\mathrm{w}}=\frac{1}{8 \pi}\left\langle\delta B^{2}\right\rangle=\frac{1}{2} \rho\left\langle\delta V^{2}\right\rangle,
$$

i.e., it is given by the mean value of the magnetic or kinetic fluctuation energy of the waves. The radial evolution of the Alfvén waves is described by the wave-energy exchange equation including wave dissipation, $Q_{\mathrm{w}}$, which may be written as

$$
V \frac{\mathrm{d}}{\mathrm{d} r} p_{\mathrm{w}}=Q_{\mathrm{w}}+\frac{1}{A} \frac{\mathrm{d}}{\mathrm{d} r}\left(A F_{\mathrm{A}}\right) .
$$

Here the Alfvén speed is $V_{\mathrm{A}}=B_{\mathrm{r}} / \sqrt{4 \pi \rho}$. The wave-energy flux density may be written as $F_{\mathrm{A}}=p_{\mathrm{w}}\left(3 V+2 V_{\mathrm{A}}\right)$. Note that $Q_{\mathrm{w}}$ is a source for thermal energy of the particles but a sink for the wave energy.

Concerning the thermodynamics and energetics of the corona and wind, there is now agreement among solar physicists that the problem of solar-wind acceleration must be treated in concert with coronal heating (Hammer 1982a, 1982b; Hollweg 1986; Hollweg and Johnson 1988; Withbroe 1988; Hansteen and Leer 1995), and that the corona cannot simply be assumed as a given boundary when dealing with the wind acceleration but rather that both phenomena are intimately connected.

For solar-wind models this requires placing the boundary not at the bottom of a $1 \mathrm{MK}$ corona but even further down in the upper chromosphere or lower transition region (Axford and McKenzie 1997; Marsch and Tu 1997a, 1997b). The price to be paid by including these complications is that radiative losses in VUV and X-ray emission and conductive losses in terms of an inward directed heat flux must be accounted for. The radiative loss function, $L=n_{\mathrm{e}}^{2} \Lambda\left(T_{\mathrm{e}}\right)$, is proportional to the squared electron density and the function $\Lambda\left(T_{\mathrm{e}}\right)$, which is given by atomic physics and determined by the collisionally excited, atomic radiation of the various highly ionized minor elements in the corona (Mariska 1992).

The coronal heating function is the great unknown in the theories of the solar corona and solar wind. Therefore, the energy input has often been modelled ad hoc (e.g., Holzer and Axford 1970; Hammer 1982a, 1982b; Withbroe 1988) and the heating has been described by a simple but convenient exponential function such that

$$
Q_{\mathrm{w}}(r)=Q_{\mathrm{w}}\left(R_{\odot}\right) \exp \left[-\left(r-R_{\odot}\right) / \lambda_{\mathrm{m}}\right]
$$

where the damping length $\lambda_{\mathrm{m}}$ is an adjustable free parameter of the model. The proton heating rate, $Q_{\mathrm{w}}$, is determined by the energy flux required to maintain the corona and wind, which in coronal holes requires the typical value of $5 \times 10^{2} \mathrm{~J} \mathrm{~m}^{-2} \mathrm{~s}^{-1}$, in compliance with the estimated overall energetics of the atmosphere and wind (see, e.g., Axford and McKenzie 1997).

In order to account for the observed temperature differences of electrons and protons, we need to consider two separate energy equations (Hartle and Sturrock 1968; Holzer and Axford 1970), in which there are different heating sources and collisional couplings between the two species. The radiative loss only appears in the electron equation, since it is the electrons that lose thermal energy by collisional VUV-line excitations and soft X-ray emissions. Usually, classical collisional conduction is considered for both species, with the thermal conductivity $\kappa_{\mathrm{e}, \mathrm{p}}$ being independent of the density $n_{\mathrm{e}, \mathrm{p}}$ and proportional to $T_{\mathrm{e}, \mathrm{p}}^{5 / 2}$ (cf., Part I, Sect. 3.1). Modified model heat-flux laws have, in the past (Hollweg 1986) and again 
recently (Hu et al. 1997), been used for the outer corona and distant wind, mainly because of our lack of understanding heat conduction processes in the collisionless solar wind in the presence of strong wave turbulence.

In order to describe the evolution of the Alfvénic fluctuations, one must consider complicated spectral transfer equations. Also, dissipation and nonlinearity should be accounted for. This forces one to consider turbulence models of the solar wind. Tu et al. (1984) and $\mathrm{Tu}(1987,1988)$ were the first to modify the Wentzel-Kramers-Brillouin (WKB) theory to describe the cascading and damping of interplanetary fluctuations and the associated heating of the solar wind. The evolution of the fluctuations is described in those papers or in Tu and Marsch (1995, 1997).

If the temperature is anisotropic, we further have to differentiate between the parallel and perpendicular degrees of freedom (e.g., Leer and Axford 1972), and consider the coupling processes between the parallel and perpendicular temperatures. Detailed parametric studies of the fast solar wind in models including the proton temperature anisotropy were carried out by Hu et al. (1997), who concluded that anisotropy has little influence on the dynamics of the solar wind, yet imposes requirements on the coronal heating mechanism.

The solar-wind acceleration mechanism will only be satisfactorily understood once progress is made in the following issues: (1) the input of mechanical energy, most likely in the form of waves and small-scale directed flows through the magnetic network, and the associated heating functions must be defined in terms of micro-physics and not simply by parametric models; (2) the transport phenomena associated with weak collisions but strong wave-particle interactions need to be described quantitatively and understood from first plasma-physics principles; and (3) the chromospheric plasma sources and the associated plasma production processes must be identified. None of the existing models fulfills these basic requirements.

\subsubsection{Models with Hot Ions in the Inner Corona}

Solar-wind models which drive the high-speed wind by wave dissipation leading to hot protons in the corona were first presented years ago by Hollweg and Johnson (1988) and Isenberg (1990). More recently, McKenzie et al. (1995) developed a theory for the highspeed solar wind with a simple dissipation-length characterization of wave heating of the coronal plasma close to the Sun, with the dissipation length $\lambda_{\mathrm{m}}$ ranging between $0.25 R_{\odot}$ and $0.5 R_{\odot}$. The significant features of the solution based on the model equations of the previous sections are: rapid acceleration with a sonic point at about $2 R_{\odot}$ and very high maximum proton temperature, namely $8 \mathrm{MK}$ to $10 \mathrm{MK}$. Such efficient near-Sun dissipation requires high-frequency Alfvén waves in the range above $1 \mathrm{~Hz}$.

Hansteen and Leer (1995) and Esser et al. (1997) presented parameter studies on the effects of very hot protons in the inner corona on the nascent solar wind. A high $T_{\mathrm{p}}$ is a genuine property of these models, and thus flow velocities of up to $800 \mathrm{~km} \mathrm{~s}^{-1}$ are readily obtained within $10 R_{\odot}$ by a deposition of heat or momentum within a fraction of a solar radius above the Sun's surface. Li et al. (1997) recently studied the influence of heavy ions on the fast wind in a three-fluid model in detail. They concluded that preferential heating of heavy ions, in particular of $\alpha$ particles, has an impact on the bulk flow that must be considered in wind models. None of the models discussed so far described the detailed physics of the heating mechanism.

\subsection{Empirical Constraints Placed by VUV Observations on the Coronal Plasma State}

Observations of the VUV line shifts from the transition region and corona are not yet conclusive as they may be due to waves and/or directed flows. Likewise, ion temperatures, 
turbulence amplitudes, oxygen differential speed, electron temperature and density in the corona ought to be clearly stated. This section reviews the empirical constraints of VUV observations on these plasma parameters.

\subsubsection{Flow Speeds Near the Sun}

One of the main objectives of SOHO was to determine the plasma state of the solar corona in order to better understand coronal heating and solar wind acceleration. SOHO measurements have placed tighter empirical constraints on the important plasma parameters in the corona. Here we will discuss them and thereby closely follow and exploit the paper by Marsch (1999). A key goal of the VUV spectrometers on SOHO was to measure by Doppler spectroscopy the initial flow velocity profiles of the fast wind emanating from coronal holes. The results were extensively discussed in Sects. 2.1.3 and 2.1.4. In the following, we will reiterate some of the key observations with respect to the question of solar wind origin. Yet, the measurement of the VUV lines emitted from plasma in the transition region and lower corona do not give a fully conclusive picture of the flow pattern there, because the shifts observed may be due to waves and/or directed flows, and the interpretation of the data is not entirely free from ambiguities.

Chae et al. (1998b) and Brekke et al. (1997a) analysed Doppler shifts from many lines for the quiet Sun and over an electron temperature range of $10^{4} \mathrm{~K}$ to $1 \mathrm{MK}$ together with more recent evaluation by Dammasch et al. (1999a, 1999b) and Peter and Judge (1999). The results are compiled in Fig. 9 in Sect. 3.3.2 and show that near $T_{\mathrm{e}}=1 \times 10^{5} \mathrm{~K}$ there is a maximum of the shift, located at about $10 \mathrm{~km} \mathrm{~s}^{-1}$ average red shift, which indicates downflows away from the observer, perhaps mostly of plasma confined in loops but not on open field lines. The interpretation of the decreasing red shift at higher temperatures is still controversial. At coronal temperatures, for Ne VIII, Mg X, and Fe XII, blue shifts have been found after a revision of the corresponding rest wavelengths.

It is only very recently that Hassler et al. (1999) have found systematic outflows (blue shifts) in the polar coronal holes and downflows (red shifts) at lower latitudes in full-Sun disk observations. They investigated in particular the blue and red shifts of the hot VUV emission line Ne VIII $77.04 \mathrm{~nm}$ and found sizeable LOS blue shifts, on the average of up to $10 \mathrm{~km} \mathrm{~s}^{-1}$, in a coronal hole in the polar cap region. Marsch and Tu (1997b) have modelled the outflow of the nascent solar wind in the coronal funnels rooted in the network lanes and got flow speeds of that order.

With the LASCO and UVCS coronagraphs the near-Sun outflow velocity profiles of the expanding corona and wind have been inferred. By taking the differences of white-light coronagraph images one can enhance the contrast and thus trace moving features in the field of view. Using this technique, Sheeley et al. (1997) derived the flow speed of the plasma in the corona. The so-identified moving coronal features were used to mark the slow wind flow. They were found to cluster at speeds around a parabolic radial path and to be characterized by a constant radial acceleration of about $4 \mathrm{~m} \mathrm{~s}^{-2}$. Habbal et al. (1997) reported velocities derived from UVCS oxygen-ion coronal images. A velocity of $100 \mathrm{~km} \mathrm{~s}^{-1}$ was observed at a distance of $1 R_{\odot}$ over the poles, whereas the acceleration was inferred to be much lower in the streamer belt, where such a value is reached only beyond $3 R_{\odot}$. Furthermore, slow solar wind seems to peel slowly off the streamer tops in an intermittent fashion (Sheeley et al. 1997), whereas evidence is accumulating that the fast wind accelerates rather rapidly.

Measurements made with the EISCAT radar facility suggested that the full acceleration of the wind over the Sun's poles occurs within $10 R_{\odot}$ (Grall et al. 1996). The direct flowvelocity measurements of UVCS clearly indicated strong near-Sun acceleration (Corti et 
al. 1997), in which the outflow speed was found to increase from $50 \mathrm{~km} \mathrm{~s}^{-1}$ at $1.5 R_{\odot}$ to $140 \mathrm{~km} \mathrm{~s}^{-1}$ at $2.3 R_{\odot}$ in the Doppler-dimming data obtained along a LOS cutting through a coronal plume.

\subsubsection{Ion Temperatures in the Corona}

The SUMER instrument is able to measure the temperature gradient from the chromosphere through the transition region into the lower corona. Seely et al. (1997) made observations in coronal streamers and found the widths of many VUV emission lines of different ions to be much larger than the thermal width expected for a kinetic temperature equal to $T_{\mathrm{e}}$ or the line formation temperature. Arguments were put forward that the effective temperature in those lines is of a kinetic nature with no or weak contribution only of a "turbulent" velocity. After Seely et al. (1997) the kinetic temperature $T_{\mathrm{i}}$ for ions originating from such elements as $\mathrm{Ne}, \mathrm{Fe}, \mathrm{Mg}$, and $\mathrm{Si}$ would range between $2.5 \mathrm{MK}$ and $6 \mathrm{MK}$ for heights above the solar limb between $100^{\prime \prime}$ and $200^{\prime \prime}$. Yet these observations are not necessarily relevant for the free-flowing solar wind.

Therefore, Tu et al. (1998) investigated the temperatures of minor ions also in a polar coronal hole, where the slits were oriented in the north-south direction and placed above the southern limb of the Sun. Figure 20 shows their data according to the mass-per-charge ratios, $m_{\mathrm{i}} / e_{\mathrm{i}}$ or $A_{\mathrm{i}} / Z_{\mathrm{i}}$ of the various ions (with $m_{\mathrm{i}}=A_{\mathrm{i}} m_{\mathrm{p}}, e_{\mathrm{i}}=Z_{\mathrm{i}} e$, and the elementary charge $e$ ). The purpose of this data presentation is to compare the different ion temperatures and discriminate them by their fundamental atomic parameters, without making assumptions on the local plasma state in the emission region.

More importantly, it was suspected that the coronal heating of the ions may be of kinetic nature and be caused primarily by cyclotron-resonance with high-frequency Alfvén waves. There is strong evidence, found by in situ observations (Marsch 1991) of the fast solar wind originating from coronal holes, for ion-cyclotron resonant heating taking place in the interplanetary medium. This possibility was for the solar wind demonstrated in some models (Marsch et al. 1982; Isenberg and Hollweg 1983), and therefore it is important to see if preferential heating occurs in the corona as well.

Figure 20 shows the ion temperatures and thermal speeds. The diamonds represent the upper limits of the temperatures and thermal speeds in the left and right panels, respectively.

Fig. 20 Minor ion temperatures and thermal speeds observed in a southern polar coronal hole. The left panel shows the common logarithm of the ion temperature, $\lg \left(T_{\mathrm{i}} / \mathrm{K}\right)$, versus the $A_{\mathrm{i}} / Z_{\mathrm{i}}$ ratio of the ions. The right panel shows the thermal speed of the ions. Full dots represent the results for $\xi=0 \mathrm{~km} \mathrm{~s}^{-1}$, while triangles are for $\xi=34 \mathrm{~km} \mathrm{~s}^{-1}$. Solid curves with plus signs refer to a value of $\xi=25 \mathrm{~km} \mathrm{~s}^{-1}$. The standard uncertainties correspond to a \pm 0.2 pixel variation of the Gaussian fit for each line (after Tu et al. 1998)
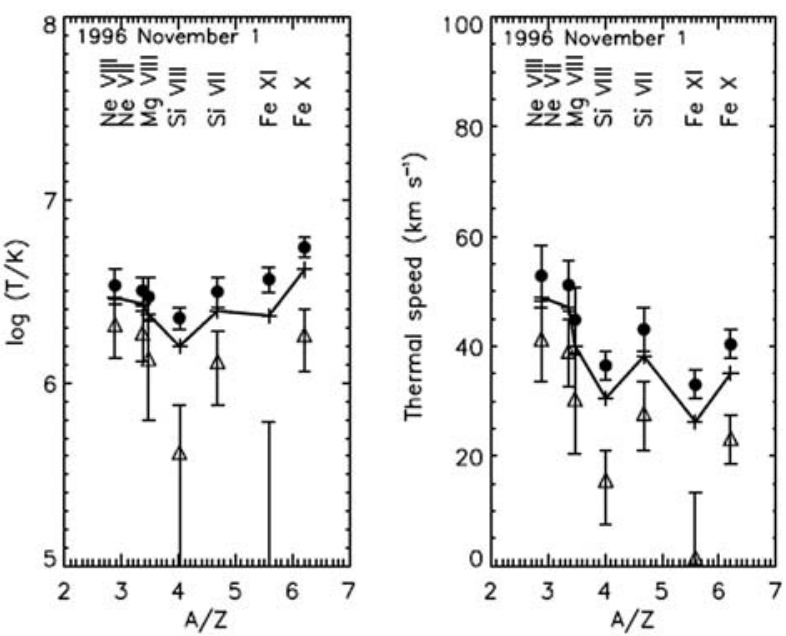
The triangles show the minimum temperatures and thermal speeds, which are calculated with a turbulent speed of $\xi=34 \mathrm{~km} \mathrm{~s}^{-1}$. This value is very near the effective speed of Fe XI, which is $37 \mathrm{~km} \mathrm{~s}^{-1}$ and equal to the maximum possible value of $\xi$. The thermal speeds seem to increase generally with the ion gyrofrequency (or decrease with $A_{\mathrm{i}} / Z_{\mathrm{i}}$ ), a result which may indicate that ion-cyclotron heating is responsible for the high ion temperatures.

\subsubsection{Turbulence Amplitudes in the Corona}

One of the key parameters from the modelling point of view, and as far as the energetics of the corona and solar wind are concerned, is the wave or turbulence amplitude. Figure 5 (see Sect. 2.1.3.1) shows the results from many authors. The turbulence amplitude $\xi$, as derived from observations on the solar disk, is given versus the line formation temperature, $T_{\mathrm{F}}$. Clearly, there seems to be an upper limit near $\xi=40 \mathrm{~km} \mathrm{~s}^{-1}$, with a decline of $\xi$ toward higher temperatures. It should be emphasized that this value would be sufficient to drive fast flows with gradual acceleration, but not flows where the acceleration is achieved essentially within the first $10 R_{\odot}$. That $\xi$ declines with height, i.e., higher formation temperatures, may be an observational bias of on-disk observations (cf., Fig. 18). Off-limb measurements (Wilhelm et al. 1998b; Tu et al. 1998) seem to indicate a further increase of the amplitude up to about $80 \mathrm{~km} \mathrm{~s}^{-1}$ near $1.35 R_{\odot}$, consistent with the expectations of WKB theory of undamped waves conserving their wave action (Jacques 1978), if we attribute the whole line width to turbulence. If the interpretation is in terms of a kinetic temperature, then Si VIII would be at $10 \mathrm{MK}$ at $1.35 R_{\odot}$.

\subsubsection{Oxygen Differential Temperature in the Corona}

All recent SOHO observations indicate that the protons, and more so the heavy ions, are rather hot in coronal holes. The minor ion temperatures are strongly anisotropic, as it is clearly indicated by the oxygen line shapes derived from the UVCS instrument which were presented by Cranmer et al. (1999b) and Kohl et al. (1997) in their reports about first results from the UVCS instrument. For oxygen, $T_{\perp}$ is much larger than $T_{\|}$, a signature which is familiar from the Helios proton in situ measurements. Some of the UVCS results are shown in Fig. 21, which gives the proton (left panel) and oxygen (right panel) temperature as a function of distance from the solar surface. We recall that the relative speed of $\mathrm{O}^{5+}$ with respect to the protons is radially increasing. Note the pronounced difference between the ion temperatures, which indicates a strong preferential heating of $\mathrm{O}^{5+}$, an effect that is interpreted as being the result of ion-cyclotron wave heating. The equivalent thermal velocity, corresponding to the Doppler width of the O VI $103.2 \mathrm{~nm}$ line, is truly remarkable because it reaches $600 \mathrm{~km} \mathrm{~s}^{-1}$. If this is interpreted as a kinetic broadening it would give a minor-ion coronal temperature of more than $100 \mathrm{MK}$ ! Strangely enough, because the Alfvén velocity is still higher, the local ion plasma $\beta$ is much smaller than unity, and thus the hot minor ions still represent a cold plasma component from the kinetic physics point of view.

\subsubsection{Coronal Electron Temperature and Density}

The electron density and temperature in coronal holes are of paramount importance, since they not only characterize the collisional properties and the electron partial pressure in the coronal hole, but also determine the ionization state of hydrogen, helium and heavier elements and govern the radiative losses in the VUV and soft X-rays through collisional line excitation (see Mariska 1992; Habbal et al. 1993). Strictly speaking it is the detailed electron 

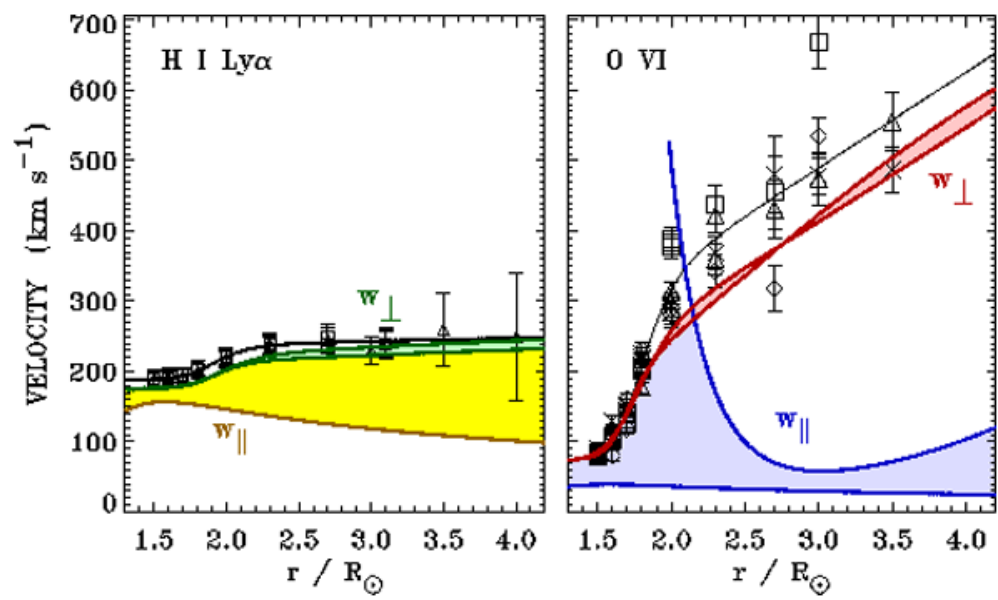

Fig. 21 The proton $(l e f t)$ and $\mathrm{O}^{5+}$ (right) ion thermal speed perpendicular $\left(W_{\perp}\right)$ and parallel $\left(W_{\|}\right)$to the magnetic field as observed in a southern polar coronal hole. Note the pronounced preferential heating of oxygen with respect to the protons, a result that perhaps indicates ion cyclotron heating (from Cranmer et al. 1999b)

distribution function that matters; this can presently not be measured close to the Sun, yet is well known farther away, where nonthermal features prevail, such as the supra-thermal tail, and the core-halo structure (for details see Pilipp et al. 1987; Marsch 1991).

Line-ratio techniques have been used to determine the electron density and to establish with SOHO the electron density profiles for the first few 100" above the limb in various regions. Wilhelm et al. (1998b) have used the Si VIII $L(144.5 \mathrm{~nm}) / L(144.0 \mathrm{~nm})$ line ratio, yielding a density that starts at $10^{8} \mathrm{~cm}^{-3}$ at $20^{\prime \prime}$ and drops to about $6 \times 10^{7} \mathrm{~cm}^{-3}$ at an altitude of $300^{\prime \prime}$ in a polar coronal hole. This profile smoothly continues into the radial profile of $n_{\mathrm{e}}$ as estimated by the UVCS measurements of oxygen O VI $L(103.2 \mathrm{~nm}) / L(103.7 \mathrm{~nm})$ line radiances and related emission measures (Corti et al. 1997). Given these densities, one can infer a corresponding flow speed of the plasma by exploiting the mass continuity equation and assuming a flow or flux tube geometry. This technique was applied by Fisher and Guhathakurta (1995) and also implies that the wind is strongly accelerated very close to the Sun.

One of the major surprises of SOHO is the fact that electrons are rather cold in coronal holes, evidence that comes from $T_{\mathrm{e}}$ estimates obtained from VUV emission-line ratios. Wilhelm et al. (1998b) used the Mg IX $75.0 \mathrm{~nm}, 70.6 \mathrm{~nm}$ line ratio and concluded that $T_{\mathrm{e}}$ hardly ever reaches the canonical value of $1 \mathrm{MK}$, but remains below it and rapidly falls off with height in the coronal hole. Similarly, David et al. (1998) derived, in a synergistic effort of the SUMER and CDS instruments using the line pair O VI $17.3 \mathrm{~nm}, 103.2 \mathrm{~nm}$, the electron temperature profiles in an equatorial streamer at the west limb and in a coronal hole at the polar north limb. Again, their observations demonstrate that electrons cool off rapidly and have only a temperature of $T_{\mathrm{e}} \approx 5 \times 10^{5} \mathrm{~K}$ at $1.3 R_{\odot}$.

These low electron temperatures are consistent with the Helios velocity distribution measurements obtained almost a full magnetic solar cycle ago, which clearly indicated that interplanetary electrons are cold and their radial temperature gradients flat in the fast streams associated with coronal holes (see the review by Marsch 1991). There also was evidence in the Helios data that interplanetary electrons cool off most strongly in fast solar wind, or 
even started at low temperatures at the Sun, a speculation at the time which now seems to be consistent with the $\mathrm{SOHO}$ results.

\section{Summary}

This review presents solar VUV observations made over the past few decades. During more than ten years of SOHO operations, and a little less for TRACE, many of new solar data were obtained. The results discussed here were mostly provided by these two unique missions. Although further results are still to be expected from this generation of space observatories, future progress in our understanding of the solar atmosphere will require new observations to be made at improved spatial and temporal resolution with novel instrumentation. ${ }^{10}$ At this stage, it appeared to us appropriate to summarize the observations obtained over the last few decades and discuss their relevance and interpretation in terms of the plasma processes and the basic plasma parameters of the solar atmosphere. We did this with emphasis on the spatial and thermal structures of the chromosphere, transition region and corona of the quiet Sun. We also discussed observations of the active Sun and of solar flares and prominences, in particular studies of the small-scale eruptive events and the associated magnetic activity. A short section is devoted to the elemental composition and abundances of the solar atmosphere. Finally, a somewhat longer section is concerned with theoretical considerations and models, where we have focused on the heating of the open corona and the origin and generation of the fast solar wind.

Acknowledgements The authors thank F.Auchère, C.M. Korendyke, M.S. Madjarska, P.C.H. Martens, H. Peter, A. Vourlidas, and T. Wang for providing information and comments. We also acknowledge the remarks and suggestions made by two anonymous referees during the review process. This research has made extensive use of the Astrophysics Data System (ADS) Bibliographic Services and of the Web of Science.

\section{References}

T. Aiouaz, M.P. Rast, Astrophys. J. 647, L183 (2006)

T. Aiouaz, H. Peter, P. Lemaire, Astron. Astrophys. 435, 713 (2005a)

T. Aiouaz, H. Peter, R. Keppens, Astron. Astrophys. 442, L35 (2005b)

T. Aiouaz, H. Peter, P. Lemaire, Astron. Astrophys. 466, 689 (2007)

S. Akiyama, G.A. Doschek, J.T. Mariska, Astrophys. J. 623, 540 (2005)

G. Alazraki, P. Couturier, Astron. Astrophys. 13, 380 (1971)

V. Aletti, M. Velli, K. Bocchialini, G. Einaudi, M. Georgoulis, J.-C. Vial, Astrophys. J. 544, 550 (2000)

M.J. Allen, H.M. Oluseyi, A.B.C. Walker, R.B. Hoover, T.W. Barbee Jr., Sol. Phys. 174, 367 (1997)

C. Allende Prieto, D.L. Lambert, M. Asplund, Astrophys. J. 556, L63 (2001)

E. Anders, N. Grevesse, Geochim. Cosmochim. Acta 53, 197 (1989)

V. Andretta, H.P. Jones, Astrophys. J. 489, 375 (1997)

V. Andretta, S.D. Jordan, J.W. Brosius, J.M. Davila, R.J. Thomas, W.E. Behring, W.T. Thompson, A. Garcia, Astrophys. J. 535, 438 (2000)

V. Andretta, G. Del Zanna, S.D. Jordan, Astron. Astrophys. 400, 737 (2003)

E. Antonucci, L. Abbo, M.A. Dodero, Astron. Astrophys. 435, 699 (2005)

E. Antonucci, M.A. Dodero, S. Giordano, Sol. Phys. 197, 115 (2000)

E. Antonucci, A.H. Gabriel, L.W. Acton, J.L. Culhane, J.G. Doyle, J.W. Leibacher, M.E. Machado, L.E. Orwig, C.G. Rapley, Sol. Phys. 78, 107 (1982)

\footnotetext{
${ }^{10}$ Two of the new solar missions mentioned in Part I, Hinode (Solar B before the launch) and the Solar Terrestrial Relations Observatory (STEREO), are now in operation, but their observations have not been considered in this review.
} 
E. Antonucci, G. Noci, J.L. Kohl, G. Tondello, M.C.E. Huber, S. Giordano, C. Benna, A. Ciaravella, S. Fineschi, L.D. Gardner, R. Martin, J. Michels, G. Naletto, P. Nicolosi, A. Panasyuk, J.C. Raymond, M. Romoli, D. Spadaro, L. Strachan, A. van Ballegooijen, ASP Conf. Ser. 118, 273 (1997)

U. Anzer, P. Heinzel, Astron. Astrophys. 446, 301 (2006)

M.J. Aschwanden, Astrophys. J. 560, 1035 (2001)

M.J. Aschwanden, Physics of the Solar Corona, An Introduction (Springer, Germany, and Praxis Publishing, Chichester, 2004)

M.J. Aschwanden, Physics of the Solar Corona. An Introduction with Problems and Solutions, 2nd edn. (Springer, New York, 2005)

M.J. Aschwanden, L. Fletcher, C.J. Schrijver, D. Alexander, Astrophys. J. 520, 880 (1999a)

M.J. Aschwanden, J.S. Newmark, J.-P. Delaboudinière, W.M. Neupert, J.A. Klimchuk, G.A. Gary, F. PortierFozzani, A. Zucker, Astrophys. J. 515, 842 (1999b)

M.J. Aschwanden, T.D. Tarbell, R.W. Nightingale, C.J. Schrijver, A. Title, C.C. Kankelborg, P. Martens, H.P. Warren, Astrophys. J. 535, 1047 (2000a)

M.J. Aschwanden, R.W. Nightingale, D. Alexander, Astrophys. J. 541, 1059 (2000b)

M.J. Aschwanden, C.J. Schrijver, D. Alexander, Astrophys. J. 550, 1036 (2001a)

M.J. Aschwanden, A.I. Poland, D.M. Rabin, Ann. Rev. Astron. Astrophys. 39, 175 (2001b)

M.J. Aschwanden, D. Alexander, N. Hurlbert, J.S. Newmark, W.M. Neupert, J.A. Klimchuk, G.A. Gary, Astrophys. J. 531, 1129 (2000c)

M.J. Aschwanden, B. De Pontieu, C.J. Schrijver, A. Title, Sol. Phys. 206, 99 (2002)

M.J. Aschwanden, R.W. Nightingale, J. Andries, M. Goossens, T. Van Doorsselaere, Astrophys. J. 598, 1375 (2003a)

M.J. Aschwanden, C.J. Schrijver, A.R. Winebarger, H.P. Warren, Astrophys. J. 588, L49 (2003b)

M. Asplund, N. Grevesse, A.J. Sauval, C. Allende Prieto, D. Kiselman, Astron. Astrophys. 417, 751 (2004)

R.G. Athay, Astrophys. J. 329, 482 (1988)

R.G. Athay, K.P. Dere, Astrophys. J. 358, 710 (1990)

R.G. Athay, O.R. White, Astrophys. J. 240, 306 (1980)

R.G. Athay, J.B. Gurman, W. Henze, R.A. Shine, Astrophys. J. 265, 519 (1983)

F. Auchère, J.W. Cook, J.S. Newmark, D.R. McMullin, R. von Steiger, M. Witte, Astrophys. J. 625, 1036 (2005)

G. Aulanier, E.E. DeLuca, S.K. Antiochos, R.A. McMullen, L. Golub, Astrophys. J. 540, 1126 (2000)

E.H. Avrett, R.L. Kurucz, R. Loeser, Astron. Astrophys. 452, 651 (2006)

W.I. Axford, J.F. McKenzie, in Solar Wind Seven, ed. by E. Marsch, R. Schwenn (Pergamon, Oxford, 1992), p. 1

W.I. Axford, J.F. McKenzie, in Cosmic Winds and the Heliosphere, ed. by J.R. Jokipii, C.P. Sonett, M.S. Giampapa (Arizona University Press, Tucson, 1997), p. 31

W.I. Axford, J.F. McKenzie, G.V. Sukhorukova, M. Banaszkiewicz, A. Czechowski, R. Ratkiewicz, Space Sci. Rev. 87, 25 (1999)

T.R. Ayres, Astrophys. J. 575, 1104 (2002)

T.R. Ayres, L. Testerman, Astrophys. J. 245, 1124 (1981)

T.R. Ayres, R.E. Stencel, J.L. Linsky, T. Simon, C. Jordan, A. Brown, O. Engvold, Astrophys. J. 274, 801 (1983)

M. Banaszkiewicz, W.I. Axford, J.F. McKenzie, Astron. Astrophys. 337, 940 (1998)

D. Banerjee, L. Teriaca, J.G. Doyle, P. Lemaire, Sol. Phys. 194, 43 (2000a)

D. Banerjee, E. O’Shea, J.G. Doyle, Sol. Phys. 196, 63 (2000b)

D. Banerjee, E. O’Shea, J.G. Doyle, M. Goossens, Astron. Astrophys. 371, 1137 (2001a)

D. Banerjee, E. O’Shea, J.G. Doyle, M. Goossens, Astron. Astrophys. 377, 691 (2001b)

J. Bartels, Terr. Magn. Atmos. Electr. 45, 339 (1940)

G.S. Basri, J.L. Linsky, J.-D.F. Bartoe, G. Brueckner, M.E. VanHoosier, Astrophys. J. 230, 924 (1979)

J. Belcher, Astrophys. J. 168, 509 (1971)

A.O. Benz, S. Krucker, Astrophys. J. 568, 413 (2002)

T.E. Berger, C.J. Schrijver, R.A. Shine, T.D. Tarbell, A.M. Title, G. Scharmer, Astrophys. J. 454, 531 (1995)

T.E. Berger, B. de Pontieu, C.J. Schrijver, A.M. Title, Astrophys. J. 519, 97 (1999)

D. Berghmans, F. Clette, D. Moses, Astron. Astrophys. 336, 1039 (1998)

D.A. Biesecker, D.C. Myers, B.J. Thompson, D.M. Hammer, A. Vourlidas, Astrophys. J. 569, 1009 (2002)

J.D. Bohlin, Sol. Phys. 51, 377 (1977)

J.D. Bohlin, N.R. Sheeley Jr., Sol. Phys. 56, 125 (1978)

J.D. Bohlin, N.R. Sheeley Jr., R. Tousey, Space Res. 15, 651 (1974)

J.D. Bohlin, S.N. Vogel, J.D. Purcell, N.R. Sheeley Jr., R. Tousey, M.E. VanHoosier, Astrophys. J. 197, L133 (1975)

B.C. Boland, S.F.T. Engstrøm, B.B. Jones, R. Wilson, Astron. Astrophys. 22, 161 (1973) 
B.C. Boland, E.P. Dyer, J.G. Firth, A.H. Gabriel, B.B. Jones, C. Jordan, R.W.P. McWhirter, P. Monk, R.F. Turner, Mon. Not. R. Astr. Soc. 171, 697 (1975)

R.M. Bonnet, E.C. Bruner Jr., L.W. Acton, W.A. Brown, M. Decaudin, Astrophys. J. 237, L47 (1980)

P. Brekke, Astrophys. J. 408, 735 (1993)

P. Brekke, Space Sci. Rev. 70, 97 (1994)

P. Brekke, Sol. Phys. 190, 379 (1999)

P. Brekke, O. Kjeldseth-Moe, Astrophys. J. 413, L55 (1994)

P. Brekke, G.J. Rottman, J. Fontenla, P.G. Judge, Astrophys. J. 468, 418 (1996)

P. Brekke, D.M. Hassler, K. Wilhelm, Sol. Phys. 175, 349 (1997a)

P. Brekke, O. Kjeldseth-Moe, R.A. Harrison, Sol. Phys. 175, 511 (1997b)

P. Brekke, N. Brynildsen, O. Kjeldseth-Moe, P. Maltby, K. Wilhelm, Adv. Space Res. 26(3), 457 (2000)

H.H. Breneman, E.C. Stone, Astrophys. J. 299, L57 (1985)

A. Brković, H. Peter, Astron. Astrophys. 406, 363 (2003)

A. Brković, H. Peter, Astron. Astrophys. 422, 709 (2004)

A. Brković, S.K. Solanki, I. Rüedi, Astron. Astrophys. 373, 1056 (2001)

A. Brković, H. Peter, S.K. Solanki, Astron. Astrophys. 403, 725 (2003)

J.W. Brosius, Astrophys. J. 622, 1216 (2005)

J.W. Brosius, E. Landi, Astrophys. J. 632, 1196 (2005)

J.W. Brosius, S.M. White, Astrophys. J. 601, 546 (2004)

J.W. Brosius, R.J. Thomas, J.M. Davila, Astrophys. J. 526, 494 (1999)

J.W. Brosius, D.M. Rabin, R.J. Thomas, Astrophys. J. 656, L41 (2007)

J.W. Brosius, J.M. Davila, W.T. Thompson, R.J. Thomas, G.D. Holman, N. Gopalswamy, S.M. White, M.R. Kundu, H.P. Jone, Astrophys. J. 411, 410 (1993)

J.W. Brosius, J.M. Davila, R.J. Thomas, B.C. Monsignori-Fossi, Astrophys. J. Suppl. Ser. 106, 143 (1996)

G.E. Brueckner, J.-D.F. Bartoe, Astrophys. J. 272, 329 (1983)

G.E. Brueckner, O.K. Moe, Space Res. XII, 1595 (1972)

G.E. Brueckner, J.-D.F. Bartoe, O. Kjeldseth-Moe, M.E. VanHoosier, Astrophys. J. 209, 935 (1976)

G.E. Brueckner, R.A. Howard, M.J. Koomen, C.M. Korendyke, D.J. Michels, J.D. Moses, D.G. Socker, K.P. Dere, P.L. Lamy, A. Llebaria, M.V. Bout, R. Schwenn, G.M. Simnett, D.K. Bedford, C.J. Eyles, Sol. Phys. 162, 357 (1995)

R. Bruno, V. Carbone, Living Rev. Sol. Phys. 2, 4 (2005)

N. Brynildsen, P. Brekke, T. Fredvik, S.V.H. Haugan, O. Kjeldseth-Moe, P. Maltby, R.A. Harrison, K. Wilhelm, Sol. Phys. 181, 23 (1998)

N. Brynildsen, P. Maltby, O. Kjeldseth-Moe, K. Wilhelm, Astrophys. J. 552, L77 (2001)

N. Brynildsen, P. Maltby, T. Fredvik, O. Kjeldseth-Moe, Sol. Phys. 207, 259 (2002)

N. Brynildsen, P. Maltby, O. Kjeldseth-Moe, K. Wilhelm, Astron. Astrophys. 398, L15 (2003)

N. Brynildsen, P. Maltby, O. Kjeldseth-Moe, K. Wilhelm, Astrophys. J. 612, 1193 (2004)

E. Buchlin, J.-C. Vial, P. Lemaire, Astron. Astrophys. 451, 1091 (2006)

F. Budnik, K.-P. Schröder, K. Wilhelm, K.-H. Glassmeier, Astron. Astrophys. 334, L77 (1998)

J. Büchner, B. Nikutowski, Proc. of the International Scientific Conference on Chromospheric and Coronal Magnetic Fields. ESA SP-596, 35.1 (2005)

J. Büchner, B. Nikutowski, A. Otto, Proc. of the SOHO 15 Workshop - Coronal Heating. ESA SP-575, 23 (2004)

J. Büchner, B. Nikutowski, A. Otto, Particle Acceleration in Astrophysical Plasmas. Geophysical Monograph Ser. 156, 161 (2005)

Bureau International des Poids et Mesures BIPM, Le système international d'unités, 8th edn. (Sèvres, France, 2005)

M. Carlsson, R.F. Stein, Astrophys. J. 440, L29 (1995)

M. Carlsson, P.G. Judge, K. Wilhelm, Astrophys. J. 486, L63 (1997)

J.H. Carver, B.H. Horton, G.W.A. Lockey, B. Rofe, Sol. Phys. 27, 347 (1972)

J. Chae, U. Schühle, P. Lemaire, Astrophys. J. 505, 957 (1998a)

J. Chae, H.S. Yun, A.I. Poland, Astrophys. J. Suppl. Ser. 114, 151 (1998b)

J. Chae, H. Wang, C.-Y. Lee, P.R. Goode, U. Schühle, Astrophys. J. 497, L109 (1998c)

J. Chae, H. Wang, C.-Y. Lee, P.R. Goode, U. Schühle, Astrophys. J. 504, 123 (1998d)

J. Chae, H. Wang, J. Qiu, P.R. Goode, K. Wilhelm, Astrophys. J. 533, 535 (2000a)

J. Chae, H. Wang, P.R. Goode, A. Fludra, U. Schühle, Astrophys. J. 528, L119 (2000b)

J. Chae, Y.-D. Park, Y.-J. Moon, H. Wang, H.S. Yun, Astrophys. J. 567, L159 (2002)

R.D. Chapman, S.D. Jordan, W.M. Neupert, R.J. Thomas, Astrophys. J. 174, L97 (1972)

S. Chapman, J. Bartels, Geomagnetism (Oxford, 1940)

P.F. Chen, M.D. Ding, C. Fang, Space Sci. Rev. 121, 201 (2005)

Y. Chen, R. Esser, Y.Q. Hu, Astrophys. J. 582, 467 (2003) 
C.-C. Cheng, E.C. Bruner, E. Tandberg-Hanssen, B.E. Woodgate, R.A. Shine, P.J. Kenny, W. Henze, G. Poletto, Astrophys. J. 253, 353 (1982)

E.G. Chipman, Sol. Phys. 55, 277 (1977)

E.G. Chipman, E.C. Bruner Jr., R.A. Shine, B.W. Lites, G.J. Rottman, R.G. Athay, O.R. White, Astrophys. J. 210, L103 (1976)

F. Chiuderi-Drago, Y. Avignon, R.J. Thomas, Sol. Phys. 51, 143 (1977)

F. Chiuderi-Drago, A. Kerdraon, E. Landi, A. Fludra, Space Sci. Rev. 87, 141 (1999)

F. Chiuderi Drago, C.E. Alissandrakis, T. Bastian, K. Bocchialini, R.A. Harrison, Sol. Phys. 199, 115 (2001)

A. Ciaravella, J.C. Raymond, B.J. Thompson, A. van Ballegooijen, L. Strachan, J. Li, L. Gardner, R. O’Neal, E. Antonucci, J. Kohl, G. Noci, Astrophys. J. 529, 575 (2000)

D. Cirigliano, J.-C. Vial, M. Rovira, Sol. Phys. 223, 95 (2004)

L. Cohen, U. Feldman, G.A. Doschek, Astrophys. J. Suppl. Ser. 37, 393 (1978)

J.W. Cook, J.A. Ewing, Astrophys. J. 355, 719 (1990)

J.W. Cook, G.E. Brueckner, J.-D.F. Bartoe, Astrophys. J. 270, L89 (1983)

G. Corti, G. Poletto, M. Romoli, J. Michels, J. Kohl, G. Noci, Proc. of the Fifth SOHO Workshop. ESA SP-404, 289 (1997)

S.R. Cranmer, Astrophys. J. 532, 1197 (2000)

S.R. Cranmer, G.B. Field, J.L. Kohl, Astrophys. J. 518, 937 (1999a)

S.R. Cranmer, J.L. Kohl, G. Noci, E. Antonucci, G. Tondello, M.C.E. Huber, L. Strachan, A.V. Panasyuk, L.D. Gardner, M. Romoli, S. Fineschi, D. Dobrzycka, J.C. Raymond, P. Nicolosi, O.H.W. Siegmund, D. Spadaro, C. Benna, A. Ciaravella, S. Giordano, S.R. Habbal, M. Karovska, X. Li, R. Martin, J.G. Michels, A. Modigliani, G. Naletto, R.H. O’Neal, C. Pernechele, G. Poletto, P.L. Smith, R.M. Suleiman, Astrophys. J. 511, 481 (1999b)

W. Curdt, P. Heinzel, Astrophys. J. 503, L95 (1998)

W. Curdt, E. Landi, U. Feldman, Astron. Astrophys. 427, 1045 (2004)

W. Curdt, E. Landi, K. Wilhelm, U. Feldman, Phys. Rev. A 62, 022502 (2000)

W. Curdt, P. Brekke, U. Feldman, K. Wilhelm, B.N. Dwivedi, U. Schühle, P. Lemaire, Astron. Astrophys. 375, 591 (2001)

G.W. Cushman, W.A. Rense, Astrophys. J. 207, L61 (1976)

G.W. Cushman, W.A. Rense, Astrophys. J. 211, L57 (1977)

L. Damé, B.H. Foing, M. Martic, M. Bruner, W. Brown, Adv. Space Res. 6, 273 (1986)

I.E. Dammasch, K. Wilhelm, Adv. Space Res. 30, 495 (2002)

I.E. Dammasch, G. Stellmacher, E. Wiehr, Astron. Nachrichten 324, 338 (2003)

I.E. Dammasch, K. Wilhelm, W. Curdt, D.M. Hassler, Astron. Astrophys. 346, 285 (1999a)

I.E. Dammasch, K. Wilhelm, W. Curdt, U. Schühle, 9th European Meeting on Solar Physics of EPS and EAS. ESA SP-448, 1165 (1999b)

I.E. Dammasch, D.M. Hassler, W. Curdt, K. Wilhelm, Space Sci. Rev. 87, 161 (1999c)

I.E. Dammasch, D.M. Hassler, K. Wilhelm, W. Curdt, Proc. of 8th SOHO Workshop. ESA SP-446, 263 (1999d)

C. David, A.H. Gabriel, F. Bely-Dubau, A. Fludra, P. Lemaire, K. Wilhelm, Astron. Astrophys. 336, L90 (1998)

C.R. de Boer, G. Stellmacher, E. Wiehr, Astron. Astrophys. 334, 280 (1998)

C.E. DeForest, J.B. Gurman, Astrophys. J. 501, L217 (1998)

C.E. DeForest, P.C. Lamy, A. Llebaria, Astrophys. J. 560, 490 (2001)

C.E. DeForest, J.T. Hoeksema, J.B. Gurman, B.J. Thompson, S.P. Plunkett, R. Howard, R.C. Harrison, D.M. Hassler, Sol. Phys. 175, 393 (1997)

C. de Jager, Bull. Astron. Inst. Neth. 17, 209 (1963)

J.-P. Delaboudinière, G.E. Artzner, J. Brunaud, A.H. Gabriel, J.-F. Hochedez, F. Millier, X.Y. Song, B. Au, K.P. Dere, R.A. Howard, R. Kreplin, D.J. Michels, J.D. Moses, J.M. Defise, C. Jamar, P. Rochus, J.P. Chauvineau, J.P. Marioge, R.C. Catura, J.R. Lemen, L. Shing, R.A. Stern, J.B. Gurman, W.M. Neupert, A. Maucherat, F. Clette, P. Cugnon, E.L. Van Dessel, Sol. Phys. 162, 291 (1995)

G. Del Zanna, B.J.I. Bromage, J. Geophys. Res. 104, 9753 (1999)

G. Del Zanna, B.J.I. Bromage, H.E. Mason, Astron. Astrophys. 398, 743 (2003)

G. Del Zanna, F. Chiuderi Drago, S. Parenti, Astron. Astrophys. 420, 307 (2004)

B. De Pontieu, T. Tarbell, R. Erdélyi, Astrophys. J. 590, 502 (2003)

K.P. Dere, Astrophys. J. 221, 1062 (1978)

K.P. Dere, H.E. Mason, Sol. Phys. 144, 217 (1993)

K.P. Dere, J.-D.F. Bartoe, G.E. Brueckner, M.D. Dykton, M.E. VanHoosier, Astrophys. J. 249, 333 (1981)

K.P. Dere, J.-D.F. Bartoe, G.E. Brueckner, Astrophys. J. 267, L65 (1983)

K.P. Dere, J.-D.F. Bartoe, G.E. Brueckner, Astrophys. J. 281, 870 (1984)

K.P. Dere, J.-D.F. Bartoe, G.E. Brueckner, J.W. Cook, D.G. Socker, Sol. Phys. 114, 223 (1987) 
K.P. Dere, J.-D.F. Bartoe, G.E. Brueckner, F. Recely, Astrophys. J. 345, L95 (1989a)

K.P. Dere, J.-D.F. Bartoe, G.E. Brueckner, Sol. Phys. 123, 41 (1989b)

K.P. Dere, J.-D.F. Bartoe, G.E. Brueckner, J. Ewing, P. Lund, J. Geophys. Res. 96, 9399 (1991)

K.P. Dere, G.E. Brueckner, R.A. Howard, D.J. Michels, J.P. Delaboudinère, Astrophys. J. 516, 465 (1999)

C.R. Detwiler, J.D. Purcell, R. Tousey, Memoires Soc. R. Sci. Liège 4, 253 (1961a)

C.R. Detwiler, D.L. Garrett, J.D. Purcell, R. Tousey, Ann. Géophys. 17, 263 (1961b)

V. Di Matteo, F. Reale, G. Peres, L. Golub, Astron. Astrophys. 342, 563 (1999)

D. Dobrzycka, A. Panasyuk, L. Strachan, J. Kohl, Space Sci. Rev. 87, 173 (1999)

D. Dobrzycka, J.C. Raymond, D.A. Biesecker, J. Li, A. Ciaravella, Astrophys. J. 588, 586 (2003)

G.A. Doschek, U. Feldman, Astrophys. J. 529, 599 (2000)

G.A. Doschek, H.P. Warren, Astrophys. J. 629, 1150 (2005)

G.A. Doschek, W.E. Behring, U. Feldman, Astrophys. J. 190, L141 (1974)

G.A. Doschek, U. Feldman, R. Tousey, Astrophys. J. 202, L151 (1975a)

G.A. Doschek, U. Feldman, K.P. Dere, G.D. Sandlin, M.E. VanHoosier, G.E. Brueckner, J.D. Purcell, R. Tousey, Astrophys. J. 196, L83 (1975b)

G.A. Doschek, U. Feldman, M.E. VanHoosier, J.-D.F. Bartoe, Astrophys. J. Suppl. Ser. 31, 417 (1976a)

G.A. Doschek, U. Feldman, J.D. Bohlin, Astrophys. J. 205, L177 (1976b)

G.A. Doschek, L. Cohen, U. Feldman, Astrophys. J. Suppl. Ser. 33, 101 (1977)

G.A. Doschek, U. Feldman, A.K. Bhatia, H.E. Mason, Astrophys. J. 226, 1129 (1978)

G.A. Doschek, R.W. Kreplin, U. Feldman, Astrophys. J. 233, L157 (1979)

G.A. Doschek, U. Feldman, R.W. Kreplin, L. Cohen, Astrophys. J. 239, 725 (1980)

G.A. Doschek, U. Feldman, R.D. Cowan, Astrophys. J. 245, 315 (1981a)

G.A. Doschek, U. Feldman, P.B. Landecker, D.L. McKenzie, Astrophys. J. 249, 372 (1981b)

G.A. Doschek, H.P. Warren, J.M. Laming, J.T. Mariska, K. Wilhelm, P. Lemaire, U. Schühle, T.G. Moran, Astrophys. J. 482, L109 (1997)

G.A. Doschek, U. Feldman, J.M. Laming, H.P. Warren, U. Schühle, K. Wilhelm, Astrophys. J. 507, 991 (1998a)

G.A. Doschek, J.M. Laming, U. Feldman, K. Wilhelm, P. Lemaire, U. Schühle, D.M. Hassler, Astrophys. J. 504, 573 (1998b)

G.A. Doschek, U. Feldman, J.M. Laming, U. Schühle, K. Wilhelm, Astrophys. J. 546, 559 (2001)

G.A. Doschek, J.T. Mariska, S. Akiyama, Astrophys. J. 609, 1153 (2004)

J.F. Dowdy, D. Rabin, R.L. Moore, Sol. Phys. 105, 35 (1986)

J.G. Doyle, M.S. Madjarska, Astron. Astrophys. 407, L29 (2003)

J.G. Doyle, H.E. Mason, J.E. Vernazza, Astron. Astrophys. 150, 69 (1985a)

J.G. Doyle, J.C. Raymond, R.W. Noyes, A.E. Kingston, Astrophys. J. 297, 816 (1985b)

J.G. Doyle, G.H.J. van den Oord, E. O’Shea, Astron. Astrophys. 327, 365 (1997)

J.G. Doyle, L. Teriaca, D. Banerjee, Astron. Astrophys. 356, 335 (2000)

J.G. Doyle, M.S. Madjarska, I. Roussev, L. Teriaca, J. Giannikakis, Astron. Astrophys. 396, 255 (2002)

J.G. Doyle, J. Giannikakis, L.D. Xia, M.S. Madjarska, Astron. Astrophys. 431, L17 (2005)

J.G. Doyle, Y. Taroyan, B. Ishak, M.S. Madjarska, S.J. Bradshaw, Astron. Astrophys. 452, 1075 (2006a)

J.G. Doyle, M.D. Popescu, Y. Taroyan, Astron. Astrophys. 446, 327 (2006b)

J.G. Doyle, B. Ishak, M.S. Madjarska, E.O. O’Shea, E. Dzifćáková, Astron. Astrophys. 451, L35 (2006c)

R.B. Dunn, J.B. Zirker, Sol. Phys. 33, 281 (1973)

P.B. Dusenbery, J.V. Hollweg, J. Geophys. Res. 86, 153 (1981)

B.N. Dwivedi, W. Curdt, K. Wilhelm, Astrophys. J. 517, 516 (1999)

B.N. Dwivedi, A. Mohan, E. Landi, in Stars as Suns: Activity, Evolution and Planets, Proc. of the 219th Symposium of the International Astronomical Union held during the IAU General Assembly XXV, Sydney, Australia, 21-25 July 2003, ed. by A.K. Dupree, A.O. Benz (San Francisco, Astronomical Society of the Pacific, 2004), p. 493

J.A. Eddy, P.J. Léna, R.M. MacQueen, Sol. Phys. 10, 330 (1969)

B. Edlén, Zeitschr. Astrophys. 22, 30 (1943)

C. Emerich, P. Lemaire, J.-C. Vial, W. Curdt, U. Schühle, K. Wilhelm, Icarus 178, 429 (2005)

R. Esser, R.J. Edgar, Astrophys. J. 563, 1055 (2001)

R. Esser, S.R. Habbal, W.A. Coles, J.V. Hollweg, J. Geophys. Res. 102, 7063 (1997)

R. Esser, S. Fineschi, D. Dobrzycka, S.R. Habbal, R.J. Edgar, J.C. Raymond, J.L. Kohl, M. Guhathakurta, Astrophys. J. 510, L63 (1999)

R. Esser, O. Lie-Svendsen, A. Janse, M.A. Killie, Astrophys. J. 629, L61 (2005)

D.A. Falconer, R.L. Moore, J.G. Porter, D.H. Hathaway, Astrophys. J. 501, 386 (1998)

U. Feldman, Astrophys. J. 275, 367 (1983)

U. Feldman, Physica Scripta 46, 202 (1992)

U. Feldman, Space Sci. Rev. 85, 227 (1998) 
U. Feldman, W.E. Behring, Astrophys. J. 189, L45 (1974)

U. Feldman, G.A. Doschek, Astrophys. J. 212, 913 (1977a)

U. Feldman, G.A. Doschek, Astrophys. J. 216, L119 (1977b)

U. Feldman, G.A. Doschek, J. Opt. Soc. Am. 67, 726 (1977c)

U. Feldman, J.M. Laming, Astrophys. J. 434, 370 (1994)

U. Feldman, J.M. Laming, Phys. Scripta 61, 222 (2000)

U. Feldman, K.G. Widing, Astrophys. J. 363, 292 (1990)

U. Feldman, K.G. Widing, Astrophys. J. 414, 381 (1993)

U. Feldman, K.G. Widing, Space Sci. Rev. 107, 665 (2003)

U. Feldman, G.A. Doschek, M.E. VanHoosier, R. Tousey, Astrophys. J. 199, L67 (1975)

U. Feldman, G.A. Doschek, M.E. VanHoosier, J.D. Purcell, Astrophys. J. Suppl. Ser. 31, 445 (1976a)

U. Feldman, G.A. Doschek, N.P. Patterson, Astrophys. J. 209, 270 (1976b)

U. Feldman, G.A. Doschek, R.W. Kreplin, Astrophys. J. 238, 365 (1980a)

U. Feldman, G.A. Doschek, R.W. Kreplin, J.T. Mariska, Astrophys. J. 241, 1175 (1980b)

U. Feldman, G.A. Doschek, L. Cohen, Astrophys. J. 255, 325 (1982)

U. Feldman, G.A. Doschek, J.A. Klimchuk, Astrophys. J. 474, 511 (1997)

U. Feldman, U. Schühle, K.G. Widing, J.M. Laming, Astrophys. J. 505, 999 (1998)

U. Feldman, G.A. Doschek, U. Schühle, K. Wilhelm, Astrophys. J. 518, 500 (1999a)

U. Feldman, K.G. Widing, H.P. Warren, Astrophys. J. 522, 1133 (1999b)

U. Feldman, I.E. Dammasch, K. Wilhelm, Space Sci. Rev. 93, 411 (2000a)

U. Feldman, W. Curdt, E. Landi, K. Wilhelm, Astrophys. J. 544, 508 (2000b)

U. Feldman, I.E. Dammasch, K. Wilhelm, P. Lemaire, D.M. Hassler, Images of the Upper Solar Atmosphere from SUMER on SOHO. ESA SP-1274 (ESA Publ. Div., Noordwijk, 2003)

U. Feldman, I.E. Dammasch, E. Landi, G.A. Doschek, Astrophys. J. 609, 439 (2004)

R. Fisher, M. Guhathakurta, Astrophys. J. 447, L139 (1995)

G.H. Fisher, R.C. Canfield, A.N. McClymont, Astrophys. J. 289, 425 (1985)

B. Fleck, F.-F. Deubner, Astron. Astrophys. 224, 245 (1989)

A. Fludra, Astron. Astrophys. 368, 639 (2001)

A. Fludra, P. Brekke, R.A. Harrison, H.E. Mason, C.D. Pike, W.T. Thompson, P.R. Young, Sol. Phys. 175, 487 (1997)

A. Fludra, G. Del Zanna, D. Alexander, B.J.I. Bromage, J. Geophys. Res. 104, 9709 (1999a)

A. Fludra, J.L.R. Saba, J.-C. Hénoux, R.J. Murphy, D.V. Reames, J.R. Lemen, K.T. Strong, J. Sylwester, K.G. Widing, The Many Faces of the Sun (Springer, New York, 1999b), p. 89

B. Foing, R.M. Bonnet, Astrophys. J. 279, 848 (1984)

C.R. Foley, S. Patsourakos, J.L. Culhane, D. MacKay, Astron. Astrophys. 381, 1049 (2002)

J.M. Fontenla, A.I. Poland, Sol. Phys. 123, 143 (1989)

P.V. Foukal, Astrophys. J. 223, 1046 (1978)

P.V. Foukal, M.C.E. Huber, R.W. Noyes, E.M. Reeves, E.J. Schmahl, J.G. Timothy, J.E. Vernazza, G.L. Withbroe, Astrophys. J. 193, L143 (1974)

M.H. Francis, R. Roussel-Dupré, Sol. Phys. 53, 465 (1977)

R.A. Frazin, S.R. Cranmer, J.L. Kohl, Astrophys. J. 597, 1145 (2003)

T. Fredvik, P. Maltby, Sol. Phys. 184, 113 (1999)

T. Fredvik, O. Kjeldseth-Moe, S.V.H. Haugan, P. Brekke, J.B. Gurman, K. Wilhelm, Adv. Space Res. 30(3), 635 (2002)

A.H. Gabriel, Phil. Trans. R. Soc. Lond. A 281, 339 (1976)

A.H. Gabriel, C. Jordan, Nature 221, 947 (1969a)

A.H. Gabriel, C. Jordan, Mon. Not. R. Astr. Soc. 145, 241 (1969b)

A.H. Gabriel, F. Bely-Dubau, P. Lemaire, Astrophys. J. 589, 623 (2003)

A.H. Gabriel, W.R.S. Garton, L. Goldberg, T.J.L. Jones, C. Jordan, F.J. Morgan, R.W. Nicholls, W.J. Parkinson, H.J.B. Paxton, E.M. Reeves, C.B. Shenton, R.J. Speer, R. Wilson, Astrophys. J. 169, 595 (1971)

A.H. Gabriel, J.L. Culhane, B.E. Patchett, E.R. Breeveld, J. Lang, J.H. Parkinson, J. Payne, K. Norman, Adv. Space Res. 15(7), 63 (1995)

A.H. Gabriel, L. Abbo, F. Bely-Dubau, A. Llebaria, E. Antonucci, Astrophys. J. 635, L185 (2005)

P.T. Gallagher, K.J.H. Phillips, L.K. Harra-Murnion, F.P. Keenan, Astron. Astrophys. 335, 733 (1998)

P.T. Gallagher, K.J.H. Phillips, L.K. Harra-Murnion, P.T. Gallagher, K.J.H. Phillips, L.K. Harra-Murnion, F. Baudin, F.P. Keenan, Astron. Astrophys. 348, 251 (1999)

K.B. Gebbie, F. Hill, J. Toomre, L.J. November, G.W. Simon, J.B. Gurman, R.A. Shine, B.E. Woodgate, R.G. Athay, C.E. Bruner Jr., R.A. Rehse, E.A. Tandberg-Hanssen, Astrophys. J. 251, L115 (1981)

J. Geiss, Space Sci. Rev. 33, 201 (1982)

S. Giordano, E. Antonucci, G. Noci, M. Romoli, J.L. Kohl, Astrophys. J. 531, L79 (2000)

V.M. Goldschmidt, Skrifter Norske Videnskapt-Akad. Oslo. Math. Naturew. K1. 4, 99 (1937) 
L. Golub, Phil. Trans. R. Soc. Lond. A 297, 595 (1980)

L. Golub, A.S. Krieger, J.W. Harvey, G.S. Vaiana, Sol. Phys. 53, 111 (1977)

L. Golub, A.S. Krieger, J.K. Silk, A.F. Timothy, G.S. Vaiana, Astrophys. J. 189, 93 (1974)

L. Golub, M. Herant, K. Kalata, I. Lovas, G. Nystrom, F. Pardo, E. Spiller, J. Wilczynski, Nature 344, 842 (1990)

L. Golub, J. Bookbinder, E. DeLuca, M. Karovska, H.P. Warren, C.J. Schrijver, R. Shine, T. Tarbell, A. Title, J. Wolfson, B. Handy, C. Kankelborg, Phys. Plasmas 6, 2205 (1999)

R.R. Grall, W.A. Coles, M.T. Klinglesmith, A.R. Breen, P.J.S. Williams, J. Markkanen, R. Esser, Lett. Nature 379, 429 (1996)

N. Grevesse, A.J. Sauval, Space Sci. Rev. 85, 161 (1998)

N. Grevesse, M. Asplund, A.J. Sauval, in Element Stratification in Stars: 40 Years of Atomic Diffusion, ed. by G. Alecian, O. Richard, S. Vauclair, EAS Publ. Ser., vol. 17 (2005), p. 21

N. Grevesse, A. Noels, A.J. Sauval, ASP Conf. Ser. 99, 117 (1996)

N.W. Griffiths, G.H. Fisher, D.T. Woods, O.H.W. Siegmund, Astrophys. J. 512, 992 (1999)

W. Grotrian, Die Naturwissenschaften 27, 214 (1939)

J.B. Gurman, J.W. Leibacher, R.A. Shine, B.E. Woodgate, W. Henze, Astrophys. J. 253, 939 (1982)

S.R. Habbal, Ann. Geophys. 10, 34 (1992)

S.R. Habbal, G.L. Withbroe, Sol. Phys. 69, 77 (1981)

S.R. Habbal, R. Esser, M.B. Arndt, Astrophys. J. 413, 435 (1993)

S.R. Habbal, R. Woo, S. Fineschi, R. O’Neil, J. Kohl, G. Noci, C. Korendyke, Astrophys. J. 489, L103 (1997)

A. Hackenberg, G. Mann, E. Marsch, Space Sci. Rev. 87, 207 (1999)

A. Hackenberg, E. Marsch, G. Mann, Astron. Astrophys. 360, 1139 (2000)

R. Hammer, Astrophys. J. 259, 767 (1982a)

R. Hammer, Astrophys. J. 259, 779 (1982b)

B.N. Handy, L.W. Acton, C.C. Kankelborg, C.J. Wolfson, D.J. Akin, M.E. Bruner, R. Caravalho, R.C. Catura, R. Chevalier, D.W. Duncan, C.G. Edwards, C.N. Feinstein, S.L. Freeland, F.M. Friedlaender, C.H. Hoffmann, N.E. Hurlburt, B.K. Jurcevich, N.L. Katz, G.A. Kelly, J.R. Lemen, M. Levay, R.W. Lindgren, D.P. Mathur, S.B. Meyer, S.J. Morrison, M.D. Morrison, R.W. Nightingale, T.P. Pope, R.A. Rehse, C.J. Schrijver, R.A. Shine, L. Shing, K.T. Strong, T.D. Tarbell, A.M. Title, D.D. Torgerson, L. Golub, J.A. Bookbinder, D. Caldwell, P.N. Cheimets, W.N. Davis, E.E. Deluca, R.A. McMullen, H.P. Warren, D. Amato, R. Fisher, H. Maldonado, C. Parkinson, Sol. Phys. 187, 229 (1999)

V.H. Hansteen, E. Leer, J. Geophys. Res. 100, 21577 (1995)

V.H. Hansteen, R. Betta, M. Carlsson, Astron. Astrophys. 360, 742 (2000)

V.H. Hansteen, E. Leer, T. Holzer, Astrophys. J. 482, 498 (1997)

L.K. Harra, P.T. Gallagher, K.J.H. Phillips, Astron. Astrophys. 362, 371 (2000)

R.A. Harrison, Astron. Astrophys. 304, 585 (1995)

R.A. Harrison, Sol. Phys. 175, 467 (1997)

R.A. Harrison, M. Lyons, Astron. Astrophys. 358, 1097 (2000)

R.A. Harrison, P. Bryans, R. Bingham, Astron. Astrophys. 379, 324 (2001)

R.A. Harrison, A.W. Hood, C.D. Pike, Astron. Astrophys. 392, 319 (2002)

R.A. Harrison, E.C. Sawyer, M.K. Carter, A.M. Cruise, R.M. Cutler, A. Fludra, R.W. Hayes, B.J. Kent, J. Lang, D.J. Parker, J. Payne, C.D. Pike, S.C. Peskett, A.G. Richards, J.L. Culhane, K. Norman, A.A. Breeveld, E.R. Breeveld, K.F. Al Janabi, A.J. McCalden, J.H. Parkinson, D.G. Self, P.D. Thomas, A.I. Poland, R.J. Thomas, W.T. Thompson, O. Kjeldseth-Moe, P. Brekke, J. Karud, P. Maltby, B. Aschenbach, H. Bräuninger, M. Kühne, J. Hollandt, O.H.W. Siegmund, M.C.E. Huber, A.H. Gabriel, H.E. Mason, B.J.I. Bromage, Sol. Phys. 162, 233 (1995)

R.A. Harrison, A. Fludra, C.D. Pike, J. Payne, W.T. Thompson, A.I. Poland, E.R. Breeveld, A.A. Breeveld, J.L. Culhane, O. Kjeldseth-Moe, M.C.E. Huber, B. Aschenbach, Sol. Phys. 170, 123 (1997)

R.A. Harrison, J. Lang, D.H. Brooks, D.E. Innes, Astron. Astrophys. 351, 1115 (1999)

R.A. Harrison, L.K. Harra, A. Brković, C.E. Parnell, Astron. Astrophys. 409, 755 (2003a)

R.A. Harrison, P. Bryans, G.M. Simnett, M. Lyons, Astron. Astrophys. 400, 1071 (2003b)

R.E. Hartle, P.A. Sturrock, Astrophys. J. 151, 1155 (1968)

D.M. Hassler, G.J. Rottman, F.W. Orrall, Astrophys. J. 372, 710 (1991)

D.M. Hassler, G.J. Rottman, E.C. Shoub, T.E. Holzer, Astrophys. J. 348, L77 (1990)

D.M. Hassler, K. Wilhelm, P. Lemaire, U. Schühle, Sol. Phys. 175, 375 (1997)

D.M. Hassler, I.E. Dammasch, P. Lemaire, P. Brekke, W. Curdt, H.E. Mason, J.C. Vial, K. Wilhelm, Science 283, 810 (1999)

P. Heinzel, B. Schmieder, J.-C. Vial, P. Kotrč, Astron. Astrophys. 370, 281 (2001a)

P. Heinzel, B. Schmieder, K. Tziotziou, Astrophys. J. 261, 223 (2001b)

N.M. Hoekzema, R.J. Rutten, J.W. Cook, Astrophys. J. 474, 518 (1997)

J.V. Hollweg, Astrophys. J. 181, 547 (1973) 
J.V. Hollweg, J. Geophys. Res. 91, 4111 (1986)

J.V. Hollweg, P.A. Isenberg, J. Geophys. Res. 107, 1147 (2002). doi:10.1029/2001JA000270

J.V. Hollweg, W. Johnson, J. Geophys. Res. 93, 9547 (1988)

T.E. Holzer, W.I. Axford, Ann. Rev. Astron. Astrophys. 8, 31 (1970)

D. Hovestadt, in Solar Wind III, ed. by C.T. Russel (University California, Los Angeles, 1974), p. 2

D. Hovestadt, M. Hilchenbach, A. Bürgi, B. Klecker, P. Laeverenz, M. Scholer, H. Grünwaldt, W.I. Axford, S. Livi, E. Marsch, B. Wilken, H.P. Winterhoff, F.M. Ipavich, P. Bedini, M.A. Coplan, A.B. Galvin, G. Gloeckler, P. Bochsler, H. Balsiger, J. Fischer, J. Geiss, R. Kallenbach, P. Wurz, K.-U. Reiche, F. Gliem, D.L. Judge, H.S. Ogawa, K.C. Hsieh, E. Möbius, M.A. Lee, G.G. Managadze, M.I. Verigin, M. Neugebauer, Sol. Phys. 162, 441 (1995)

Y.Q. Hu, R. Esser, S.R. Habbal, J. Geophys. Res. 102, 14661 (1997)

M.C.E. Huber, P.V. Foukal, R.W. Noyes, E.M. Reeves, E.J. Schmahl, J.G. Timothy, J.E. Vernazza, G.L. Withbroe, Astrophys. J. 194, L115 (1974)

C.L. Hyder, B.W. Lites, Sol. Phys. 14, 147 (1970)

D.E. Innes, Astron. Astrophys. 378, 1067 (2001)

D.E. Innes, Proc. of SOHO 13. ESA SP-378, 215 (2004)

D.E. Innes, D.E. McKenzie, T. Wang, Sol. Phys. 217, 247 (2003)

D.E. Innes, B. Inhester, W.I. Axford, K. Wilhelm, Nature 386, 811 (1997a)

D.E. Innes, P. Brekke, D. Germerott, K. Wilhelm, Sol. Phys. 175, 341 (1997b)

D.E. Innes, W. Curdt, R. Schwenn, S. Solanki, G. Stenborg, D.E. McKenzie, Astrophys. J. 549, L249 (2001)

P.A. Isenberg, J. Geophys. Res. 95, 6437 (1990)

P.A. Isenberg, J.V. Hollweg, J. Geophys. Res. 88, 3923 (1983)

S.A. Jacques, Astrophys. J. 226, 632 (1978)

C. Jordan, Mon. Not. R. Astr. Soc. 170, 429 (1975)

C. Jordan, G.E. Brueckner, J.-D.F. Bartoe, G.D. Sandlin, M.E. VanHoosier, Astrophys. J. 226, 687 (1978)

P.G. Judge, M. Carlsson, R.F. Stein, Astrophys. J. 597, 1158 (2003)

P.G. Judge, M. Carlsson, K. Wilhelm, Astrophys. J. 490, L195 (1997)

P.G. Judge, T.D. Tarbell, K. Wilhelm, Astrophys. J. 554, 424 (2001)

P.G. Judge, V. Hansteen, Ø. Wikstøl, K. Wilhelm, U. Schühle, T. Moran, Astrophys. J. 502, 981 (1998)

W. Kalkofen, P. Ulmschneider, E.H. Avrett, Astrophys. J. 521, 141 (1999)

C.C. Kankelborg, A.B.C. Walker Jr., R.B. Hoover, T.W. Barbee Jr., Astrophys. J. 466, 529 (1996)

R. Kano, S. Tsuneta, PASJ 48, 535 (1996)

F.P. Keenan, A.E. Kingston, P.L. Dufton, J.G. Doyle, K.G. Widing, Sol. Phys. 94, 91 (1984)

F.P. Keenan, A.C. Katsiyannis, K.M. Aggarwal, M. Mathioudakis, J.W. Brosius, J.M. Davila, R.J. Thomas, Sol. Phys. 212, 65 (2003)

C.F. Kennel, F. Engelmann, Phys. Fluids 9, 2377 (1966)

O. Kjeldseth-Moe, P. Brekke, in B. Schmieder, A. Hofmann, J. Staude (eds.), ASP Conf. Ser. 184 (1999) p. 286

O. Kjeldseth-Moe, C.C. Cheng, Space Sci. Rev. 70, 85 (1994)

O. Kjeldseth Moe, K.R. Nicolas, Astrophys. J. 211, 579 (1977)

A. Klassen, H. Aurass, G. Mann, B.J. Thompson, Astron. Astrophys. Suppl. 141, 357 (2000)

B. Kliem, I.E. Dammasch, W. Curdt, K. Wilhelm, Astrophys. J. 568, L61 (2002)

Y.-K. Ko, L.A. Fisk, J. Geiss, G. Gloeckler, M. Guhathakurta, Sol. Phys. 171, 345 (1997)

Y.-K. Ko, J.C. Raymond, T.H. Zurbuchen, P. Riley, J.M. Raines, L. Strachan, Astrophys. J. 646, 1275 (2006)

J.L. Kohl, G.L. Withbroe, Astrophys. J. 256, 263 (1982)

J.L. Kohl, L. Strachan, L.D. Gardner, Astrophys. J. 465, L141 (1996)

J.L. Kohl, H. Weiser, G.L. Withbroe, R.W. Noyes, W.H. Parkinson, E.M. Reeves, R.H. Munro, R.M. MacQueen, Astrophys. J. 241, L117 (1980)

J.L. Kohl, L.D. Gardner, L. Strachan, D.M. Hassler, Space Sci. Rev. 70, 253 (1994)

J.L. Kohl, L.D. Gardner, L. Strachan, R. Fisher, M. Guhathakurta, Space Sci. Rev. 72, 29 (1995a)

J.L. Kohl, R. Esser, L.D. Gardner, S. Habbal, P.S. Daigneau, E.F. Dennis, G.U. Nystrom, A. Panasyuk, J.C. Raymond, P.L. Smith, L. Strachan, A.A. Van Ballegooijen, G. Noci, S. Fineschi, M. Romoli, A. Ciaravella, A. Modigliani, M.C.E. Huber, E. Antonucci, C. Benna, S. Giordano, G. Tondello, P. Nicolosi, G. Naletto, C. Pernechele, D. Spadaro, G. Poletto, S. Livi, O. Von der Lühe, J. Geiss, J.G. Timothy, G. Gloeckler, A. Allegra, G. Basile, R. Brusa, B. Wood, O.H.W. Siegmund, W. Fowler, R. Fisher, M. Jhabvala, Sol. Phys. 162, 313 (1995b)

J.L. Kohl, G. Noci, E. Anonucci, G. Tondello, M.C.E. Huber, L.D. Gardner, P. Nicolosi, L. Strachan, S. Fineschi, J.C. Raymond, M. Romoli, D. Spadaro, A. Panasyuk, O.H.W. Siegmund, C. Benna, A. Ciaravella, S.R. Cranmer, S. Giordano, M. Karovska, R. Martin, J. Michels, A. Modigliani, G. Naletto, C. Pernechele, G. Poletto, P.L. Smith, Sol. Phys. 170, 613 (1997) 
J.L. Kohl, G. Noci, E. Antonucci, G. Tondello, M.C.E. Huber, S.R. Cranmer, L. Strachan, A. Panasyuk, L.D. Gardner, M. Romoli, S. Fineschi, D. Dobrzycka, J.C. Raymond, P. Nicolosi, O.H.W. Siegmund, D. Spadaro, C. Benna, A. Ciaravella, S. Giordano, S.R. Habbal, M. Karovska, X. Li, R. Martin, J.G. Michels, A. Modigliani, G. Naletto, R.H. O’Neal, G. Pernechele, C. Poletto, P.L. Smith, R.M. Suleiman, Astrophys. J. 501, L127 (1998)

J.L. Kohl, R. Esser, S.R. Cranmer, S. Fineschi, L.D. Gardner, A.V. Panasyuk, L. Strachan, R.M. Suleiman, R.A. Frazin, G. Noci, Astrophys. J. 510, L59 (1999)

J.L. Kohl, G. Noci, S.R. Cranmer, J.C. Raymond, Astron. Astrophys. Rev. 13, 31 (2006)

C.M. Korendyke, A. Vourlidas, J.W. Cook, K.P. Dere, R.A. Howard, J.S. Morrill, J.D. Moses, N.E. Moulton, D.G. Socker, Sol. Phys. 200, 63 (2001)

M. Kretzschmar, J. Lilensten, J. Aboudarham, Astron. Astrophys. 419, 345 (2004)

A.S. Krieger, A.F. Timothy, E.C. Roelof, Sol. Phys. 29, 505 (1973)

J.M. Krijger, R.J. Rutten, B.W. Lites, Th. Straus, R.A. Shine, T.D. Tarbell, Astron. Astrophys. 379, 1052 (2001)

T.A. Kucera, V. Andretta, A.I. Poland, Sol. Phys. 183, 107 (1998)

R. Lallement, J.C. Raymond, J. Vallerga, M. Lemoine, F. Dalaudier, J.L. Bertaux, Astron. Astrophys. 426, 875 (2004a)

R. Lallement, J.C. Raymond, J.-L. Bertaux, E. Quémerais, Y.-K. Ko, M. Uzzo, D. McMullin, D. Rucinski, Astron. Astrophys. 426, 867 (2004b)

J.M. Laming, U. Feldman, Astrophys. J. 403, 434 (1993)

J.M. Laming, U. Feldman, Astrophys. J. 546, 552 (2001)

J.M. Laming, J.J. Drake, K.G. Widing, Astrophys. J. 443, 416 (1995)

J.M. Laming, U. Feldman, U. Schühle, P. Lemaire, W. Curdt, K. Wilhelm, Astrophys. J. 485, 911 (1997)

E. Landi, U. Feldman, K.P. Dere, Astrophys. J. Suppl. Ser. 139, 281 (2002)

E. Landi, U. Feldman, G.A. Doschek, Astrophys. J. 643, 1258 (2006)

E. Landi, U. Feldman, G.A. Doschek, Astrophys. J. 659, 743 (2007)

E. Landi, H.E. Mason, P. Lemaire, M. Landini, Astron. Astrophys. 357, 743 (2000)

E. Leer, W.I. Axford, Sol. Phys. 23, 238 (1972)

R.B. Leighton, R.W. Noyes, G.W. Simon, Astrophys. J. 135, 474 (1962)

P. Lemaire, K. Wilhelm, W. Curdt, U. Schühle, E. Marsch, S.D. Poland, A.I. Jordan, R.J. Thomas, D.M. Hassler, J.-C. Vial, M. Kühne, O.H.W. Huber, M.C.E. Siegmund, A. Gabriel, J.G. Timothy, M. Grewing, Sol. Phys. 170, 105 (1997)

P. Lemaire, C. Emerich, W. Curdt, U. Schühle, K. Wilhelm, Astron. Astrophys. 334, 1095 (1998)

P. Lemaire, K. Bocchialini, V. Aletti, D.M. Hassler, K. Wilhelm, Space Sci. Rev. 87, 249 (1999)

P. Lemaire, P. Gouttebroze, J.-C. Vial, W. Curdt, U. Schühle, K. Wilhelm, Astron. Astrophys. 418, 737 (2004)

P. Lemaire, C. Emerich, J.-C. Vial, W. Curdt, U. Schühle, K. Wilhelm, Adv. Space Res. 35, 384 (2005)

D.D. Lenz, E.E. DeLuca, L. Golub, R. Rosner, J.A. Bookbinder, Astrophys. J. 517, L155 (1999)

X. Li, R. Esser, S.R. Habbal, Y.-Q. Hu, J. Geophys. Res. 102, 17419 (1997)

X. Li, S.R. Habbal, J.L. Kohl, G. Noci, Astrophys. J. 501, L133 (1998)

B.W. Lites, in Sunspots: Theory and Observations, ed. by J.H. Thomas, N.O. Weiss (Kluwer, Dordrecht, 1992), p. 261

B.W. Lites, E.C. Bruner Jr., E.G. Chipman, R.A. Shine, G.J. Rottman, O.R. White, R.G. Athay, Astrophys. J. 210, L111 (1976)

R. Lüst, L. Biermann, E. Trefftz, F. Meyer, Z. Naturforschung A17, 259 (1962)

B. Lyot, L'Astronomie 51, 203 (1937)

P. MacNeice, R. Pallavicini, H.E. Mason, G.M. Simnett, E. Antonucci, R.A. Shine, D.M. Rust, C. Jordan, B.R. Dennis, Sol. Phys. 99, 167 (1985)

K.P. Macpherson, C. Jordan, Mon. Not. R. Astr. Soc. 308, 510 (1999)

M.S. Madjarska, J.G. Doyle, Astron. Astrophys. 403, 731 (2003)

M.S. Madjarska, J.G. Doyle, L. van Driel-Geiztelyi, Astrophys. J. 603, L57 (2004)

M.S. Madjarska, J.G. Doyle, L. Teriaca, D. Banerjee, Astron. Astrophys. 398, 775 (2003)

M.S. Madjarska, J.G. Doyle, J.-F. Hochedez, A. Theissen, Astron. Astrophys. 452, L11 (2006)

P. Maltby, N. Brynildsen, O. Kjeldseth-Moe, K. Wilhelm, Astron. Astrophys. 373, L1 (2001)

J.T. Mariska, Astrophys. J. 225, 252 (1978)

J.T. Mariska, Astrophys. J. 235, 268 (1980)

J.T. Mariska, The Solar Transition Region. Cambridge Astrophysics Series, vol. 23 (University Press, Cambridge, 1992)

J.T. Mariska, Astrophys. J. 434, 756 (1994)

J.T. Mariska, G.A. Doschek, U. Feldman, Astrophys. J. 232, 929 (1979)

J.T. Mariska, U. Feldman, G.A. Doschek, Astrophys. J. 226, 698 (1978)

E. Marsch, in Physics of the Inner Heliosphere, ed. by R. Schwenn, E. Marsch, vol. 2 (Springer, 1994), p. 45 
E. Marsch, Space Sci. Rev. 87, 1 (1999)

E. Marsch, in The Sun and the Heliosphere as an Integrated System, ed. by G. Poletto, S.T. Suess (Kluwer, Dordrecht, 2004), p. 283

E. Marsch, in Solar Influence on the Heliosphere and Earth's Environment: Recent Progress and Prospects, ed. by N. Gopalswamy, A. Bhattacharyya. Proceedings of the ILWS Workshop Goa, India: February 19-24, 2006, Quest Publications for ILWS and Indian Institute of Geomagnetism, 2006a, p. 111

E. Marsch, Adv. Space Res. 38, 921 (2006b)

E. Marsch, C.Y. Tu, Astron. Astrophys. 319, L17 (1997a)

E. Marsch, C.-Y. Tu, Sol. Phys. 176, 87 (1997b)

E. Marsch, C.Y. Tu, J. Geophys. Res. 106, 227 (2001a)

E. Marsch, C.Y. Tu, J. Geophys. Res. 106, 8357 (2001b)

E. Marsch, W.I. Axford, J.M. McKenzie, in The Dynamic Sun, ed. by B.N. Dwivedi (Cambridge University Press, Cambridge, 2003), p. 374

E. Marsch, C.K. Goertz, K. Richter, J. Geophys. Res. 87, 5030 (1982)

E. Marsch, C.Y. Tu, K. Wilhelm, Astron. Astrophys. 359, 381 (2000)

E. Marsch, T. Wiegelmann, L.D. Xia, Astron. Astrophys. 428, 629 (2004)

E. Marsch, C.Y. Tu, K. Wilhelm, W. Curdt, U. Schühle, I.E. Dammasch, ESA SP 404, 555 (1997)

E. Marsch, C.-Y. Tu, P. Heinzel, K. Wilhelm, W. Curdt, Astron. Astrophys. 347, 676 (1999)

E. Marsch, G.-Q. Zhou, J.-S. He, C.-Y. Tu, Astron. Astrophys. 457, 699 (2006)

P.C.H. Martens, C.C. Kankelborg, T.E. Berger, Astrophys. J. 537, 471 (2000)

M. Martic, L. Damé, M.E. Bruner, B.H. Foing, Adv. Space Res. 11, 241 (1991)

H.E. Mason, Adv. Space Res. 15(7), 53 (1995)

D.J. McComas, S.J. Bame, B.L. Barraclough, W.C. Feldman, H.O. Funsten, J.T. Gosling, P. Riley, R. Skoug, A. Balogh, R. Forsyth, B.E. Goldstein, M. Neugebauer, Geophys. Res. Lett. 25, 1 (1998)

S.W. McIntosh, P.G. Judge, Astrophys. J. 561, 420 (2001)

S.W. McIntosh, A.R. Davey, D.M. Hassler, Astrophys. J. 664, L87 (2006)

S.W. McIntosh, T.J. Bogdan, P.S. Cally, M. Carlsson, V.H. Hansteen, P.G. Judge, B.W. Lites, H. Peter, C.S. Rosenthal, T.D. Tarbell, Astrophys. J. 548, L237 (2001)

J.F. McKenzie, H.S. Hudson, Astrophys. J. 519, 93 (1999)

J.F. McKenzie, M. Banaszkiewicz, W.I. Axford, Astron. Astrophys. 303, L45 (1995)

D.R. McMullin, M. Bzowski, E. Möbius, A. Pauluhn, R. Skoug, W.T. Thompson, M. Witte, R. von Steiger, D. Rucinski, D. Judge, M. Banaszkiewicz, R. Lallement, Astron. Astrophys. 426, 885 (2004)

P. Mein, Sol. Phys. 54, 45 (1977)

J.E. Mendoza-Torres, J.P. Torres-Papaqui, K. Wilhelm, Astron. Astrophys. 431, 339 (2005)

J.-P. Meyer, Astrophys. J. Suppl. Ser. 57, 173 (1985)

J.P. Meyer, in ASP Cosmic Abundances, ed. by S.S. Holt, G. Sonneborn. ASP Conf. Ser., vol. 99, 1996, p. 127

S.R. Miralles, M.P. Cranmer, J.L. Kohl, Adv. Space Res. 33, 696 (2004)

S.R. Miralles, M.P., Cranmer, A.V. Panasyuk, M. Romoli, J.L. Kohl, Astrophys. J. 549, L257 (2001)

R.L. Moore, D.A. Falconer, J.G. Porter, S.T. Suess, Astrophys. J. 526, 505 (1999)

G.F. Moreton, S \& T 21, 145 (1961)

D. Moses, J.W. Cook, J.-D.F. Bartoe, G.E. Brueckner, K.P. Dere, D.F. Webb, J.M. Davis, J.W. Harvey, F. Recely, S.F. Martin, H. Zirin, Astrophys. J. 430, 913 (1994)

T. Mouschovias, A.I. Poland, Astrophys. J. 220, 672 (1978)

R.H. Munro, G.L. Withbroe, Astrophys. J. 176, 511 (1972)

R.H. Munro, J.T. Gosling, R.M. Hildner, E. MacQueen, A.I. Poland, C.L. Ross, Sol. Phys. 61, 201 (1979)

V.M. Nakariakov, in Dynamic Sun, ed. by B.N. Dwivedi (Cambridge University Press, Cambridge, 2003), p. 314

V.M. Nakariakov, L. Ofman, Astron. Astrophys. 372, L53 (2001)

M. Neugebauer, Science 252, 404 (1991)

W.M. Neupert, J.W. Brosius, R.J. Thomas, W.T. Thompson, Astrophys. J. 392, L95 (1992)

K.R. Nicolas, O. Kjeldseth-Moe, J.-D.F. Bartoe, G.E. Brueckner, Sol. Phys. 81, 253 (1982)

G. Noci, J.L. Kohl, G.L. Withbroe, Astropyhs. J. 315, 706 (1987)

G. Noci, J.L. Kohl, E. Antonucci, G. Tondello, M.C.E. Huber, S. Fineschi, L.D. Gardner, C.M. Korendyke, P. Nicolosi, M. Romoli, D. Spadaro, L. Maccari, J.C. Raymond, O.H.W. Siegmund, C. Benna, A. Ciaravella, S. Giordano, J. Michels, A. Modigliani, G. Naletto, A. Panasyuk, C. Pernechele, G. Poletto, P.L. Smith, L. Strachan, Proc. of the Fifth SOHO Workshop. ESA SP-404, 75 (1997)

R.W. Noyes, J.C. Raymond, J.G. Doyle, A.E. Kingston, Astrophys. J. 297, 805 (1985)

L. Ofman, T. Wang, Astrophys. J. 514, 441 (2002)

L. Ofman, V.M. Nakariakov, C.E. DeForest, Astrophys. J. 514, 441 (1999)

H.S. Ogawa, D.R. Judge, D.R. McMullin, P. Gangopadhyay, A.B. Galvin, J. Geophys. Res. 103, 1 (1998) 
T.J. Okamoto, H. Nakai, A. Keiyama, N. Narukage, S. UeNo, R. Kitai, H. Kurokawa, K. Shibata, Astrophys. J. 608, 1124 (2004)

R.H. O’Neal Jr., Thesis, Stanford University, 1995

R. O’Neal, A.B.C. Walker Jr., R.B. Hoover, T.W. Barbee Jr., SPD 24.1717 (1993) (Abstract)

E. O’Shea, D. Banerjee, J.G. Doyle, Astron. Astrophys. 436, L43 (2005)

E. O’Shea, K. Muglach, B. Fleck, Astron. Astrophys. 387, 642 (2002)

E. O’Shea, D. Banerjee, J.G. Doyle, B. Fleck, F. Murtagh, Astron. Astrophys. 368, 1095 (2001)

S.P. Owocki, J.D. Scudder, Astrophys. J. 270, 758 (1983)

I. Pagano, J.L. Linsky, J. Valenti, D.K. Duncan, Astron. Astrophys. 415, 331 (2004)

S. Parenti, B.J.I. Bromage, G. Poletto, G. Noci, J.C. Raymond, G.E. Bromage, Astron. Astrophys. 363, 800 (2000)

S. Parenti, B.J.I. Bromage, G.E. Bromage, Astron. Astrophys. 384, 303 (2002)

S. Parenti, J.-C. Vial, P. Lemaire, Sol. Phys. 220, 61 (2004)

S. Parenti, P. Lemaire, J.-C. Vial, Astron. Astrophys. 443, 685 (2005a)

S. Parenti, J.-C. Vial, P. Lemaire, Astron. Astrophys. 443, 679 (2005b)

E.N. Parker, Astrophys. J. 128, 664 (1958)

E.N. Parker, Astrophys. J. 264, 642 (1983)

E.N. Parker, Astrophys. J. 330, 474 (1988)

W.H. Parkinson, E.M. Reeves, Sol. Phys. 10, 342 (1969)

C.E. Parnell, P.E. Jupp, Astrophys. J. 529, 554 (2000)

S. Patsourakos, J.-C. Vial, Astron. Astrophys. 359, L1 (2000)

S. Patsourakos, J.-C. Vial, Sol. Phys. 208, 253 (2002)

S. Patsourakos, S.R. Habbal, Y.Q. Hu, Astrophys. J. 581, L125 (2002)

S. Patsourakos, J.-C. Vial, A.H. Gabriel, N. Bellamine, Astrophys. J. 522, 540 (1999)

G. Peres, F. Reale, L. Golub, Astrophys. J. 422, 412 (1994)

H. Peter, Astrophys. J. 516, 490 (1999a)

H. Peter, Astrophys. J. 522, L77 (1999b)

H. Peter, Astron. Astrophys. 360, 761 (2000)

H. Peter, Astron. Astrophys. 374, 1108 (2001)

H. Peter, Astron. Astrophys. 449, 759 (2006)

H. Peter, A. Brković, Astron. Astrophys. 403, 287 (2003)

H. Peter, P. Judge, Astrophys. J. 522, 1148 (1999)

H. Peter, E. Marsch, Proc. of the Fifth SOHO Workshop. ESA SP-404, 591 (1997)

C.D. Pike, R.A. Harrison, Sol. Phys. 175, 457 (1997)

C.D. Pike, H.E. Mason, Sol. Phys. 206, 359 (2002)

H.E. Phillips, K.J.H. Bhatia, A.K. Mason, D.M. Zarro, Astrophys. J. 466, 519 (1996)

J. Phillips, K.J.H. Sylwester, B. Sylwester, E. Landi, Astrophys. J. 589, L113 (2003)

W.G. Pilipp, H. Miggenrieder, M.D. Montgomery, K.-H. Mühlhäuser, H. Rosenbauer, R. Schwenn, J. Geophys. Res. 92, 1075 (1987)

G.W. Pneuman, R.A. Kopp, Sol. Phys. 57, 49 (1978)

A.I. Poland, E. Tandberg-Hanssen, Sol. Phys. 84, 63 (1983)

M.D. Popescu, J.G. Doyle, L.D. Xia, Astron. Astrophys. 421, 339 (2004)

J.G. Porter, J. Toomre, K.B. Gebbie, Astrophys. J. 283, 879 (1984)

J.G. Porter, O. Moore, R.L. Reichmann, E.J. Engvold, K.L. Harvey, Astrophys. J. 323, 380 (1987)

N.-E. Raouafi, S.K. Solanki, Astron. Astrophys. 412, 271 (2003)

N.-E. Raouafi, S.K. Solanki, Astron. Astrophys. 427, 725 (2004)

N.-E. Raouafi, S. Sahal-Bréchot, P. Lemaire, V. Bommier, Astron. Astrophys. 390, 691 (2002)

B. Ravindra, P. Venkatakrishnan, Sol. Phys. 214, 267 (2003)

J.C. Raymond, J.G. Doyle, Astrophys. J. 247, 686 (1981)

J.C. Raymond, J.L. Kohl, G. Noci, E. Antonucci, G. Tondello, M.C.E. Huber, L.D. Gardner, P. Nicolosi, S. Fineschi, M. Romoli, D. Spadaro, O.H.W. Siegmund, C. Benna, A. Ciaravella, S.R. Cranmer, S. Giordano, M. Karovska, R. Martin, J. Michels, A. Modigliani, G. Naletto, A. Panasyuk, G. Pernechele, C. Poletto, P.L. Smith, R.M. Suleiman, L. Strachan, Sol. Phys. 175, 645 (1997)

J.C. Raymond, A. Ciaravella, D. Dobrzycka, L. Strachan, Y.-K. Ko, M. Uzzo, N.-E. Raouafi, Astrophys. J. 597, 1106 (2003)

F. Reale, G. Peres, Astrophys. J. 528, L45 (2000)

S. Redfield, J.L. Linsky, T.B. Ake, T.R. Ayres, A.K. Dupree, R.D. Robinson, B.E. Wood, P.R. Young, Astrophys. J. 581, 626 (2002)

E.M. Reeves, Sol. Phys. 46, 53 (1976)

E.M. Reeves, P.V. Foukal, M.C.E. Huber, R.W. Noyes, E.J. Schmahl, J.G. Timothy, J.E. Vernazza, G.L. Withbroe, Astrophys. J. 188, L27 (1974) 
P. Riley, J.A. Linker, Z. Mikić, J. Geophys. Res. 106, 15889 (2001). doi:10.1029/2000JA000121

E. Robbrecht, E. Verwichte, D. Berghmans, J.F. Hochedez, S. Poedts, V.M. Nakariakov, Astron. Astrophys. 370, 591 (2001)

B. Roberts, Solar Variability: From Core to Outer Frontiers. ESA SP-506, 481 (2002)

B. Roberts, Proc. of SOHO 13. ESA SP-547, 1 (2003)

B. Roberts, V.M. Nakariakov, in Turbulence, Waves, and Instabilities in the Solar Plasma. NATO Science Series (Kluwer, 2003), p. 165

B. Roberts, P.M. Edwin, A.O. Benz, Astrophys. J. 279, 857 (1984)

B. Rompolt, Sol. Phys. 41, 329 (1975)

F.D. Rosenberg, U. Feldman, G.A. Doschek, Astrophys. J. 212, 905 (1977)

G.J. Rottman, F.Q. Orrall, J.A. Klimchuk, Astrophys. J. 260, 326 (1982)

H.N. Russell, Astrophys. J. 70, 11 (1929)

R.J. Rutten, J.M. Krijger, Astron. Astrophys. 407, 735 (2003)

J.L.R. Saba, Adv. Space Res. 15(7), 13 (1995)

D. Samain, R.M. Bonnet, R. Gayet, C. Lizambert, Astron. Astrophys. 39, 71 (1975)

P.H. Scherrer, R.S. Bogart, R.I. Bush, J.T. Hoeksema, A.G. Kosovichev, J. Schou, W. Rosenberg, L. Springer, T.D. Tarbell, A. Title, C.J. Wolfson, I. Zayer, the MDI Engineering Team, Sol. Phys. 162, 129 (1995)

E.J. Schmahl, F.Q. Orrall, Astrophys. J. 231, L41 (1979)

J.T. Schmelz, J.L.R. Saba, K.T. Strong, H.D. Winter, J.W. Brosius, Astrophys. J. 523, 432 (1999)

J.T. Schmelz, K. Nasraoui, G. Del Zanna, J.W. Cirtain, E.E. DeLuca, H.E. Mason, Astrophys. J. 658, L119 (2007a)

J.T. Schmelz, V.L. Kashyap, M.A. Weber, Astrophys. J. 660, L157 (2007b)

B. Schmieder, Y. Lin, P. Heinzel, P. Schwartz, Sol. Phys. 221, 297 (2004)

C.J. Schrijver, M.J. Aschwanden, Astrophys. J. 566, 1147 (2002)

C.J. Schrijver, A.M. Title, H.J. Hagenaar, R.A. Shine, Sol. Phys. 175, 329 (1997)

C.J. Schrijver, A.M. Title, K.L. Harvey, N.R. Sheeley Jr., Y.M. Wang, G.H.J. van den Oord, R.A.D. Shine, T. Tarbell, N.E. Hulburt, Nature 394, 152 (1998)

C.J. Schrijver, A.M. Title, T.E. Berger, L. Fletcher, N.E. Hurlburt, R.W. Nightingale, R.A. Shine, T.D. Tarbell, J. Wolfson, L. Golub, J.A. Bookbinder, E.E. DeLuca, R.A. McMullen, H.P. Warren, C.C. Kankelborg, B.N. Handy, B. De Pontieu, Sol. Phys. 187, 261 (1999)

C.J. Schrijver, M.J. Aschwanden, A.M. Title, Sol. Phys. 206, 69 (2002)

U. Schühle, C.M. Brown, W. Curdt, U. Feldman, Proc. of SOHO 8. ESA SP-446, 617 (1999)

R. Schwenn, J.C. Raymond, D. Alexander, A. Ciaravella, N. Gopalswamy, R. Howard, H. Hudson, P. Kaufmann, A. Klassen, D. Maia, G. Munoz-Martinez, M. Pick, M. Reiner, N. Srivastava, D. Tripathi, A. Vourlidas, Y.-M. Wang, J. Zhang, Space Sci. Rev. 123, 127 (2006). doi:10.1007/s11214-006-9016-y

J.D. Scudder, Astrophys. J. 398, 299 (1992)

J.F. Seely, U. Feldman, Mon. Not. R. Astr. Soc. 213, 417 (1985)

J.F. Seely, U. Feldman, U. Schühle, K. Wilhelm, W. Curdt, P. Lemaire, Astrophys. J. 484, L87 (1997)

N.R. Sheeley Jr., L. Golub, Sol. Phys. 63, 119 (1979)

N.R. Sheeley Jr., Y.-M. Wang, S.H. Hawley, G.E. Brueckner, K.P. Dere, R.A. Howard, M.J. Koomen, C.M. Korendyke, D.J. Michels, S.E. Paswaters, D.G. Socker, O.C. St. Cyr, D. Wang, P.L. Lamy, A. Llebaria, R. Schwenn, G.M. Simnett, S. Plunkett, D.A. Biesecker, Astrophys. J. 484, 472 (1997)

R.A. Shine, D. Roussel-Dupre, E.C. Bruner Jr., E.G. Chipman, B.W. Lites, G.J. Rottman, R.G. Athay, O.R. White, Astrophys. J. 210, L107 (1976)

K. Shibata, T. Yokoyama, M. Shimojo, Adv. Space Res. 17, 197 (1996)

S.K. Solanki, A. Lagg, J. Woch, N. Krupp, M. Collados, Nature 425, 692 (2003)

D. Spadaro, A.C. Lanzafame, L. Consoli, E. Marsch, D.H. Brooks, J. Lang, Astron. Astrophys. 359, 716 (2000)

G. Stellmacher, E. Wiehr, I.E. Dammasch, Sol. Phys. 217, 133 (2003)

A.C. Sterling, Sol. Phys. 196, 79 (2000)

A.C. Sterling, H.S. Hudson, B.J. Thompson, D.M. Zarro, Astrophys. J. 532, 628 (2000)

L. Strachan, R. Suleiman, A.V. Panasyuk, D.A. Biesecker, J.L. Kohl, Astrophys. J. 571, 1008 (2002)

J. Sýkora, O.G. Badalyan, V.N. Obridko, Adv. Space Res. 29(3), 395 (2002)

J. Sylwester, I. Gaicki, Z. Kordylewski, M. Kowalinski, S. Nowak, S. Plocieniak, M. Siarkowski, B. Sylwester, W. Trzebinski, J. Bakala, J.L. Culhane, M. Whyndham, R.D. Bentley, P.R. Guttridge, K.J.H. Phillips, J. Lang, C.M. Brown, G.A. Doschek, V.D. Kuznetsov, V.N. Oraevsky, A.I. Stepanov, D.V. Lisin, Sol. Phys. 226, 45 (2005)

E. Tandberg-Hanssen, The Nature of Solar Prominences. Astrophys. Space Sci. Library, vol. 199 (Kluwer, Dordrecht, 1995)

L. Teriaca, M.S. Madjarska, J.G. Doyle, Sol. Phys. 200, 91 (2001)

L. Teriaca, M.S. Madjarska, J.G. Doyle, Astron. Astrophys. 392, 309 (2002a) 
L. Teriaca, A. Falchi, J.G. Doyle, M.S. Madjarska, D. Banerjee, Proc. of 10th European Solar Physics Meeting. ESA SP-506, 777 (2002b)

L. Teriaca, G. Poletto, M. Romoli, D. Biesecker, Astrophys. J. 588, 566 (2003)

L. Teriaca, D. Banerjee, A. Falchi, J.G. Doyle, M.S. Madjarska, Astron. Astrophys. 427, 1065 (2004)

P. Testa, G. Peres, F. Reale, S. Orlando, Astrophys. J. 580, 1159 (2002)

R.J. Thomas, W.M. Neupert, Astrophys. J. Suppl. Ser. 91, 461 (1994)

B.J. Thompson, S.P. Plunkett, J.B. Gurman, J.S. Newmark, O.C. St.Cyr, D.J. Michels, Geophys. Res. Lett. 25, 2465 (1998)

B.J. Thompson, J.B. Gurman, W.M. Neupert, J.S. Newmark, J.-P. Delaboudinière, O.C. St.Cyr, S. Stezelberger, K.P. Dere, R.A. Howard, D.J. Michels, Astrophys. J. 517, L151 (1999)

B.J. Thompson, E.W. Cliver, N. Nitta, C. Delannée, J.-P. Delaboundinière, Geophys. Res. Lett. 27, 1431 (2000)

A.F. Timothy, A.S. Krieger, G.S. Vaiana, Sol. Phys. 42, 135 (1975)

R. Tousey, Q. J. R. Astron. Soc. 5, 123 (1964)

R. Tousey, J.-D.F. Bartoe, J.D. Bohlin, G.E. Brueckner, J.D. Purcell, V.E. Scherrer, N.R. Shelley Jr., R.J. Schumacher, M.E. VanHoosier, Sol. Phys. 33, 265 (1973)

R. Tousey, J.-D.F. Bartoe, G.E. Brueckner, J.D. Purcell, Appl. Opt. 16, 870 (1977)

S. Tsuneta, L. Acton, M. Bruner, J. Jemen, W. Brown, R. Caravalho, R. Catura, S. Freeland, B. Jurcevich, M. Morrison, Y. Ogawara, T. Hirayama, J. Owens, Sol. Phys. 136, 37 (1991)

C.-Y. Tu, Sol. Phys. 109, 149 (1987)

C.-Y. Tu, J. Geophys. Res. 93, 7 (1988)

C.-Y. Tu, E. Marsch, MHD structures, waves and turbulence in the solar wind. Space Sci. Rev. 73, 1 (1995)

C.-Y. Tu, E. Marsch, Sol. Phys. 171, 363 (1997)

C.-Y. Tu, E. Marsch, J. Geophys. Res. 106, 8233 (2001a)

C.-Y. Tu, E. Marsch, Astron. Astrophys. 368, 1071 (2001b)

C.-Y. Tu, Z.-Y. Pu, F.-S. Wei, J. Geophys. Res. 89, 9695 (1984)

C.-Y. Tu, E. Marsch, K. Wilhelm, W. Curdt, Astrophys. J. 503, 475 (1998)

C.-Y. Tu, E. Marsch, K. Wilhelm, Space Sci. Rev. 87, 331 (1999)

C.-Y. Tu, C. Zhou, E. Marsch, L.-D. Xia, L. Zhao, J.-X. Wang, K. Wilhelm, Science 308, 519 (2005a)

C.-Y. Tu, C. Zhou, E. Marsch, K. Wilhelm, L. Zhao, L.-D. Xia, J.-X. Wang, Astrophys. J. 624, L133 (2005b)

Y. Uchida, Sol. Phys. 4, 30 (1968)

I. Ugarte-Urra, J.G. Doyle, G. Del Zanna, Astron. Astrophys. 435, 1169 (2005)

A. Unsöld, Z. Astrophys. 24, 306 (1948)

H.C. van de Hulst, Bull. Astron. Inst. Neth. 11, 135 (1950a)

H.C. Van de Hulst, Bull. Astron. Inst. Neth. 11, 150 (1950b)

A.M. Vásquez, J.C. Raymond, Astrophys. J. 619, 1132 (2005)

J.E. Vernazza, E.H. Avrett, R. Loeser, Astrophys. J. Suppl. Ser. 45, 635 (1981)

J.E. Vernazza, P.V. Foukal, M.C.E. Huber, R.W. Noyes, E.M. Reeves, E.J. Schmahl, J.G. Timothy, G.L. Withbroe, Astrophys. J. 199, L123 (1975)

A. Vidal-Madjar, Sol. Phys. 40, 69 (1975)

O. Vilhu, J. Huovelin, S. Pohjolainen, J. Virtanen, W. Curdt, Astron. Astrophys. 395, 977 (2002)

C. Vocks, E. Marsch, Geophys. Res. Lett. 28, 1917 (2001)

C. Vocks, E. Marsch, Astrophys. J. 568, 1030 (2002)

R. von Steiger, J.-C. Vial, P. Bochsler, M. Chaussidon, C.M.S. Cohen, B. Fleck, V.S. Heber, H. Holweger, K. Issautier, A.J. Lazarus, K.W. Ogilvie, J.A. Paquette, D.B. Reisenfeld, L. Teriaca, K. Wilhelm, S. Yusainee, J.M. Laming, R.C. Wiens, AIPC 598, 13 (2001)

A. Vourlidas, J.A. Klimchuk, C.M. Korendyke, T.D. Tarbell, B.N. Handy, Astrophys. J. 563, 374 (2001)

M. Waldmeier, Die Sonnenkorona I (Birkhäuser, Basel, 1951)

M. Waldmeier, Die Sonnenkorona II (Birkhäuser, Basel, 1957)

A.B.C. Walker Jr., T.W. Barbee Jr., R.B. Hoover, J.F. Lindblom, Science 241, 1781 (1988)

H. Wang, Astrophys. J. 509, 461 (1998)

T.J. Wang, Proc. of SOHO 13. ESA SP-547, 417 (2004)

T.J. Wang, S.K. Solanki, Astron. Astrophys. 421, L33 (2004)

T.J. Wang, S.K. Solanki, W. Curdt, D.E. Innes, I.E. Dammasch, Astrophys. J. 574, L101 (2002)

T.J. Wang, S.K. Solanki, D.E. Innes, W. Curdt, E. Marsch, Astron. Astrophys. 402, L17 (2003)

Y.-M. Wang, Astrophys. J. 501, L145 (1998)

Y.-M. Wang, Astrophys. J. 520, L71 (1999)

Y.-M. Wang, Astrophys. J. 560, 456 (2001)

Y.-M. Wang, N.R. Sheeley Jr., Astrophys. J. 355, 726 (1990) 
Y.-M. Wang, N.R. Sheeley Jr., K.P. Dere, R.T. Duffin, R.A. Howard, D.J. Michels, J.D. Moses, J.W. Harvey, D.D. Branston, J.-P. Delaboudinière, G.E. Artzner, J.F. Hochedez, J.M. Defise, R.C. Catura, J.R. Lemen, J.B. Gurman, W.M. Neupert, J. Newmark, B. Thompson, A. Maucherat, F. Clette, Astrophys. J. 484, L75 (1997)

Y.M. Wang, N.R. Sheeley Jr., J.H. Walters, G.E. Brueckner, R.A. Howard, D.J. Michels, P.L. Lamy, R. Schwenn, G.M. Simnett, Astrophys. J. 498, L165 (1998)

H.P. Warren, Sol. Phys. 190, 393 (1999)

H.P. Warren, G.A. Doschek, Astrophys. J. 618, L157 (2005)

H.P. Warren, D.M. Hassler, J. Geophys. Res. 104, 9781 (1999)

H.P. Warren, A.D. Warshall, Astrophys. J. 571, 999 (2002)

H.P. Warren, A.R. Winebarger, Astrophys. J. 596, L113 (2003)

H.P. Warren, J.T. Mariska, K. Wilhelm, P. Lemaire, Astrophys. J. 484, L91 (1997a)

H.P. Warren, J.T. Mariska, K. Wilhelm, Astrophys. J. 490, L187 (1997b)

H.P. Warren, J.T. Mariska, K. Wilhelm, Astrophys. J. Suppl. Ser. 119, 105 (1998)

D.F. Webb, S.F. Martin, D. Moses, J.W. Harvey, Sol. Phys. 144, 15 (1993)

K.G. Widing, U. Feldman, Astrophys. J. 344, 1046 (1989)

K.G. Widing, U. Feldman, Astrophys. J. 442, 446 (1995)

K.G. Widing, U. Feldman, Astrophys. J. 555, 426 (2001)

K.G. Widing, J.D. Purcell, G.D. Sandlin, Sol. Phys. 12, 52 (1970)

K.G. Widing, U. Feldman, A.K. Bhatia, Astrophys. J. 308, 982 (1986)

K.G. Widing, E. Landi, U. Feldman, Astrophys. J. 622, 1211 (2005)

T. Wiegelmann, T. Neukirch, Sol. Phys. 208, 233 (2002)

T. Wiegelmann, S.K. Solanki, Sol. Phys. 225, 227 (2004)

T. Wiegelmann, L.D. Xia, E. Marsch, Astron. Astrophys. 432, L1 (2005)

E. Wiehr, B. Bovelet, J. Hirzberger, Astron. Astrophys. 422, L63 (2004)

K. Wilhelm, in Proc. of Third SOLTIP Symp., ed. by X.S. Feng, F.S. Wei, M. Dryer (Academic, 1998), p. 75

K. Wilhelm, Astrophys. Space Sci. 264, 43 (1999)

K. Wilhelm, Adv. Space Res. 25, 1723 (2000a)

K. Wilhelm, Astron. Astrophys. 360, 351 (2000b)

K. Wilhelm, J. Atmos. Terr. Sol. Phys. 65, 167 (2003)

K. Wilhelm, Astron. Astrophys. 455, 697 (2006)

K. Wilhelm, R. Bodmer, Space Sci. Rev. 85, 371 (1998)

K. Wilhelm, W. Kalkofen, Astron. Astrophys. 408, 1137 (2003)

K. Wilhelm, W. Curdt, E. Marsch, U. Schühle, P. Lemaire, A.H. Gabriel, J.-C. Vial, M. Grewing, M.C.E. Huber, S.D. Jordan, A.I. Poland, R.J. Thomas, M. Kühne, J.G. Timothy, D.M. Hassler, O.H.W. Siegmund, Sol. Phys. 162, 189 (1995)

K. Wilhelm, P. Lemaire, W. Curdt, U. Schühle, E. Marsch, A.I. Poland, S.D. Jordan, R.J. Thomas, D.M. Hassler, M.C.E. Huber, J.-C. Vial, M. Kühne, O.H.W. Siegmund, A.H. Gabriel, J.G. Timothy, M. Grewing, U. Feldman, J. Hollandt, P. Brekke, Sol. Phys. 170, 75 (1997)

K. Wilhelm, P. Lemaire, I.E. Dammasch, J. Hollandt, U. Schühle, W. Curdt, T. Kucera, D.M. Hassler, M.C.E. Huber, Astron. Astrophys. 334, 685 (1998a)

K. Wilhelm, E. Marsch, B.N. Dwivedi, D.M. Hassler, P. Lemaire, A. Gabriel, M.C.E. Huber, Astrophys. J. 500, 1023 (1998b)

K. Wilhelm, D.E. Innes, W. Curdt, B. Kliem, P. Brekke, Solar Jets and Coronal Plumes. ESA SP-421, 103 (1998c)

K. Wilhelm, T.N. Woods, U. Schühle, W. Curdt, P. Lemaire, G.J. Rottman, Astron. Astrophys. 352, 321 (1999)

K. Wilhelm, I.E. Dammasch, E. Marsch, D.M. Hassler, Astron. Astrophys. 353, 749 (2000)

K. Wilhelm, B. Inhester, J.S. Newmark, Astron. Astrophys. 382, 328 (2002a)

K. Wilhelm, I.E. Dammasch, D.M. Hassler, Astrophys. Space Sci. 282, 189 (2002b)

K. Wilhelm, B.N. Dwivedi, E. Marsch, U. Feldman, Space Sci. Rev. 111, 415 (2004a), Part I

K. Wilhelm, B.N. Dwivedi, L. Teriaca, Astron. Astrophys. 415, 1133 (2004b)

K. Wilhelm, A. Fludra, L. Teriaca, R.A. Harrison, B.N. Dwivedi, C.D. Pike, Astron. Astrophys. 435, 733 (2005a)

K. Wilhelm, U. Schühle, W. Curdt, M. Hilchenbach, E. Marsch, P. Lemaire, J.-L. Bertaux, S.D. Jordan, U. Feldman, Astron. Astrophys. 439, 701 (2005b)

A.R. Winebarger, A.G. Emslie, J.T. Mariska, H.P. Warren, Astrophys. J. 565, 1298 (2002a)

A.R. Winebarger, A.C. Updike, K.K. Reeves, Astrophys. J. 570, L105 (2002b)

A.R. Winebarger, H.P. Warren, A. van Ballegooijen, E.E. DeLuca, L. Golub, Astrophys. J. 567, L89 (2002c)

A.R. Winebarger, H.P. Warren, J.T. Mariska, Astrophys. J. 587, 439 (2003)

G.L. Withbroe, Astrophys. J. 325, 442 (1988) 
G.L. Withbroe, R.W. Noyes, Ann. Rev. Astron. Astrophys. 15, 363 (1977)

G.L. Withbroe, J.L. Kohl, H. Weiser, R.H. Munro, Space Sci. Rev. 33, 17 (1982)

J. Woch, W.I. Axford, U. Mall, B. Wilken, S. Livi, J. Geiss, G. Gloeckler, R.J. Forsyth, Geophys. Res. Lett. 24, 2885 (1997)

R. Woo, J. Martin, Geophys. Res. Lett. 24, 2535 (1997)

R. Woo, S.R. Habbal, U. Feldman, Astrophys. J. 612, 1171 (2004)

B.E. Wood, J.L. Linsky, T.R. Ayres, Astrophys. J. 478, 745 (1997)

T.N. Woods, W.K. Tobiska, G.J. Rottman, J.R. Worden, J. Geophys. Res. 105, 27195 (2000)

L. Xia, Thesis, University Göttingen, Copernicus GmbH, Katlenburg-Lindau, 2003

L. Xia, E. Marsch, W. Curdt, Astron. Astrophys. 399, L5 (2003)

L. Xia, E. Marsch, K. Wilhelm, Astron. Astrophys. 424, 1025 (2004)

L. Xia, M.D. Popescu, J.G. Doyle, J. Giannikakis, Astron. Astrophys. 438, 1115 (2005)

T. Yokoyama, K. Akita, T. Morimoto, K. Inoue, J. Newmark, Astrophys. J. 546, 69 (2001)

P.R. Young, Astron. Astrophys. 439, 361 (2005)

P.R. Young, H.E. Mason, Sol. Phys. 175, 523 (1997)

P.R. Young, H.E. Mason, Space Sci. Rev. 85, 315 (1998)

P.R. Young, J.A. Klimchuk, H.E. Mason, Astron. Astrophys. 350, 286 (1999)

P.R. Young, F.R. Mason, H.E. Keenan, K.G. Widing, Astron. Astrophys. 323, 243 (1997)

V.B. Yurchyshyn, Astrophys. J. 576, 493 (2002)

D.M. Zarro, A.C. Sterling, B.J. Thompson, H.S. Hudson, N. Nitta, Astrophys. J. 520, L139 (1999)

M. Zhang, L. Golub, Astrophys. J. 595, 1251 (2003)

J. Zhang, M.R. Kundu, S.M. White, Sol. Phys. 198, 347 (2001a)

J. Zhang, K.P. Dere, R.A. Howard, M.R. Kundu, S.M. White, Astrophys. J. 559, 452 (2001b)

J.T. Zhao, X.P. Hoeksema, P.H. Scherrer, J. Geophys. Res. 104, 9735 (1999)

H. Zirin, Astrophysics of the Sun (Cambridge University Press, Cambridge, 1988) 